Supporting Information for

\title{
N-(2-Nitrophenyl)proline: An Intramolecular Hydrogen Bond Forming Reagent for the Determination of the Absolute Configuration of Primary Amines
}

Hee Choon Ahn and Kihang Choi

\section{(S)-N-(2-nitrophenyl)proline ((S)- 3)}

L-Proline (1.05 g, $9.0 \mathrm{mmol}$ ) was dissolved in $50 \mathrm{~mL} \mathrm{H} \mathrm{H}_{2} \mathrm{O} / 50 \mathrm{~mL}$ EtOH. To the solution was added $\mathrm{NaHCO}_{3}$ (1.90 g, 2.5 eq.), followed by 1-fluoro-2-nitrobenzene (0.96 mL, 1.0 eq.). The resulting mixture was refluxed for $5 \mathrm{hr}$ and then cooled down to room temperature. After removing EtOH by evaporation, the remaining solution was acidified $(\mathrm{pH} 2-3)$ with 1 $\mathrm{N} \mathrm{HCl}$ and extracted with EtOAc (x3). The combined organic layers were washed with brine, dried over anhydrous $\mathrm{MgSO}_{4}$, and concentrated under reduced pressure. The residue was dried under vacuum to give, in quantitative yield, a yellow oil that solidified on standing. m.p. $86^{\circ} \mathrm{C} ;[\alpha]_{\mathrm{D}}{ }^{25}=-1080(c=1.0$ in $\mathrm{MeOH}) ;{ }^{1} \mathrm{H}$ NMR $\left(400 \mathrm{MHz}, \mathrm{CDCl}_{3}, 25^{\circ} \mathrm{C}\right.$, TMS): $\delta=7.74\left(\mathrm{dd},{ }^{3} J(\mathrm{H}, \mathrm{H})=8.1 \mathrm{~Hz},{ }^{4} J(\mathrm{H}, \mathrm{H})=1.7 \mathrm{~Hz}, 1 \mathrm{H}\right), 7.40\left(\mathrm{ddd},{ }^{3} J(\mathrm{H}, \mathrm{H})=8.5 \mathrm{~Hz}\right.$, $\left.{ }^{3} J(\mathrm{H}, \mathrm{H})=7.2 \mathrm{~Hz}, \quad{ }^{4} J(\mathrm{H}, \mathrm{H})=1.7 \mathrm{~Hz}, \quad 1 \mathrm{H}\right), 6.90 \quad\left(\mathrm{~d},{ }^{3} J(\mathrm{H}, \mathrm{H})=8.5 \mathrm{~Hz}, 1 \mathrm{H}\right), 6.88 \quad$ (ddd, $\left.{ }^{3} J(\mathrm{H}, \mathrm{H})=8.1 \mathrm{~Hz},{ }^{3} J(\mathrm{H}, \mathrm{H})=7.2 \mathrm{~Hz},{ }^{4} J(\mathrm{H}, \mathrm{H})=1.0 \mathrm{~Hz}, 1 \mathrm{H}\right), 4.42-4.45(\mathrm{~m}, 1 \mathrm{H}), 3.53-3.59(\mathrm{~m}$, $1 \mathrm{H}), 3.03-3.08(\mathrm{~m}, 1 \mathrm{H}), 2.46-2.54(\mathrm{~m} ., 1 \mathrm{H}), 2.05-2.22(\mathrm{~m}, 2 \mathrm{H}), 1.89-2.00 \mathrm{ppm}(\mathrm{m}, 1 \mathrm{H}) ;{ }^{13} \mathrm{C}$ NMR (100 MHz, $\mathrm{CDCl}_{3}, 25^{\circ} \mathrm{C}$, TMS): $\delta=178.5,141.3,138.8,133.3,126.7,118.0,116.8$, 61.8, 51.8, 31.0, 24.9 ppm; IR $\left(\mathrm{CCl}_{4}\right)$ : 2927, $1721(\mathrm{C}=\mathrm{O}) \mathrm{cm}^{-1}$; HRMS(FAB) Cald. for $\mathrm{C}_{11} \mathrm{H}_{12} \mathrm{~N}_{2} \mathrm{O}_{4} \mathrm{Na}\left[\mathrm{M}+\mathrm{Na}^{+}\right]: 259.0695$, Found: 259.0699 .

\section{(R)- $N$-(2-nitrophenyl)proline ( $(R)$ - 3)}

The procedure described for $(S)-3$ was followed except that D-proline was used instead of L-proline. The spectral data were virtually identical to those of $(S)-3$ except $[\alpha]_{\mathrm{D}}^{25}=+1020$ $(c=1.0$ in $\mathrm{MeOH})$.

\section{(S)- $N$-(4-nitrophenyl)proline ((S)- 4)}

The procedure described for $(S)-3$ was followed except that 1-fluoro-4-nitrobenzene was used instead of 1-fluoro-2-nitrobenzene. yield $93 \%$ m.p. $150^{\circ} \mathrm{C} ;[\alpha]_{\mathrm{D}}{ }^{25}=-293(c=1.0$ in $\mathrm{MeOH}) ;{ }^{1} \mathrm{H}$ NMR $\left(400 \mathrm{MHz}, \mathrm{CDCl}_{3}, 25^{\circ} \mathrm{C}, \mathrm{TMS}\right): \delta=8.13\left(\mathrm{~d},{ }^{3} \mathrm{~J}(\mathrm{H}, \mathrm{H})=9.2 \mathrm{~Hz}, 2 \mathrm{H}\right), 6.51(\mathrm{~d}$, 
$\left.{ }^{3} J(\mathrm{H}, \mathrm{H})=9.2 \mathrm{~Hz}, 2 \mathrm{H}\right), 4.40\left(\mathrm{dd},{ }^{3} J(\mathrm{H}, \mathrm{H})=8.3 \mathrm{~Hz},{ }^{3} J(\mathrm{H}, \mathrm{H})=2.7 \mathrm{~Hz}, 1 \mathrm{H}\right), 3.61-3.67(\mathrm{~m}, 1 \mathrm{H})$, 3.45-3.51 (m, 1H), 2.29-2.45 (m, 2H), 2.13-2.23 ppm (m, 2H); ${ }^{13} \mathrm{C} \mathrm{NMR} \mathrm{(100} \mathrm{MHz,} \mathrm{CDCl}_{3}$, $\left.25^{\circ} \mathrm{C}, \mathrm{TMS}\right): \delta=178.9,151.0,137.9,126.3,111.1,60.5,48.6,30.8,23.6 \mathrm{ppm}$; IR $\left(\mathrm{CCl}_{4}\right)$ : 2927, $1716(\mathrm{C}=\mathrm{O}) \mathrm{cm}^{-1}$; HRMS(FAB) Cald. for $\mathrm{C}_{11} \mathrm{H}_{12} \mathrm{~N}_{2} \mathrm{O}_{4} \mathrm{Na}\left[\mathrm{M}+\mathrm{Na}^{+}\right]: 259.0695$, Found: 259.0692.

\section{(R)- $N$-(4-nitrophenyl)proline ((R)- 4)}

The procedure described for $(S)$-3 was followed except that D-proline and 1-fluoro-4nitrobenzene were used instead of L-proline and 1-fluoro-2-nitrobenzene, respectively. The spectral data were virtually identical to those of $(S)-4$ except $[\alpha]_{\mathrm{D}}{ }^{25}=+301(c=1.0$ in $\mathrm{MeOH})$.

\section{Synthesis of 2- or 4-NPP amides: a general procedure}

$(S)$ - or $(R)$-3 (50 mg, $0.21 \mathrm{mmol}$ ) and bis(2-oxo-3-oxazolidinyl)-phosphinic chloride (BOP$\mathrm{Cl}, 61 \mathrm{mg}, 1.1$ eq.) were dissolved in $2 \mathrm{~mL} \mathrm{CH}_{2} \mathrm{Cl}_{2}$. To the solution was added a primary amine (1.0 eq.) followed by $N, N$-diisopropylethylamine $(0.13 \mathrm{~mL}, 3.5$ eq. $)$. After stirring overnight, the mixture was diluted with EtOAc and washed with $0.1 \mathrm{~N} \mathrm{HCl}$, saturated $\mathrm{NaHCO}_{3}$ solution, and brine. The organic layer was dried over $\mathrm{MgSO}_{4}$ and concentrated under reduced pressure. The residue was purified by $\mathrm{SiO}_{2}$ chromatography to give a 2-NPP amide in $50-98 \%$ yield.

(S)-2-NPP amide of L-leucine methyl ester $(5, \mathrm{R}=(S)$-2-NPP)

$[\alpha]_{\mathrm{D}}{ }^{25}=-1170(c=1.0$ in $\mathrm{MeOH}) ;{ }^{1} \mathrm{H}$ NMR $\left(400 \mathrm{MHz}, \mathrm{CDCl}_{3}, 25^{\circ} \mathrm{C}, \mathrm{TMS}\right): \delta=7.79$ (dd, $\left.{ }^{3} J(\mathrm{H}, \mathrm{H})=8.1 \mathrm{~Hz}, \quad{ }^{4} J(\mathrm{H}, \mathrm{H})=1.7 \mathrm{~Hz}, \quad 1 \mathrm{H}\right), 7.43 \quad\left(\mathrm{ddd}, \quad{ }^{3} J(\mathrm{H}, \mathrm{H})=8.5 \mathrm{~Hz}, \quad{ }^{3} J(\mathrm{H}, \mathrm{H})=7.2 \mathrm{~Hz}\right.$, $\left.{ }^{4} J(\mathrm{H}, \mathrm{H})=1.7 \mathrm{~Hz}, 1 \mathrm{H}\right), 7.07\left(\right.$ br d, $\left.{ }^{3} J(\mathrm{H}, \mathrm{H})=8.1 \mathrm{~Hz}, 1 \mathrm{H} ; \mathrm{NH}\right), 7.04\left(\mathrm{dd},{ }^{3} J(\mathrm{H}, \mathrm{H})=8.5 \mathrm{~Hz}\right.$, $\left.{ }^{3} J(\mathrm{H}, \mathrm{H})=1.0 \mathrm{~Hz}, 1 \mathrm{H}\right), 6.94\left(\mathrm{ddd},{ }^{3} J(\mathrm{H}, \mathrm{H})=8.1 \mathrm{~Hz},{ }^{3} J(\mathrm{H}, \mathrm{H})=7.2 \mathrm{~Hz},{ }^{4} J(\mathrm{H}, \mathrm{H})=1.0 \mathrm{~Hz}, 1 \mathrm{H}\right)$, 4.39-4.63 (m, 2H), 3.6-3.74 (m, 1H), $3.46(\mathrm{~s}, 3 \mathrm{H}), 2.81-2.87(\mathrm{~m} ., 1 \mathrm{H}), 2.53-2.65(\mathrm{~m}, 1 \mathrm{H})$, $1.82-2.09(\mathrm{~m}, 3 \mathrm{H}), 1.50-1.63(\mathrm{~m}, 3 \mathrm{H}), 0.94\left(\mathrm{~d},{ }^{3} J(\mathrm{H}, \mathrm{H})=6.2 \mathrm{~Hz}, 3 \mathrm{H}\right), 0.92 \mathrm{ppm}(\mathrm{d}$, $\left.{ }^{3} J(\mathrm{H}, \mathrm{H})=6.2 \mathrm{~Hz}, 3 \mathrm{H}\right)$; IR $\left(\mathrm{CCl}_{4}\right): 3377(\mathrm{NH}), 1750,1634(\mathrm{C}=\mathrm{O}) \mathrm{cm}^{-1}$; MS(FAB): $m / z 386.3$ $\left[\mathrm{M}+\mathrm{Na}^{+}\right]$.

( $R$ )-2-NPP amide of L-leucine methyl ester $(5, \mathrm{R}=(R)$-2-NPP) $[\alpha]_{\mathrm{D}}{ }^{25}=+861(c=1.0$ in $\mathrm{MeOH}) ;{ }^{1} \mathrm{H}$ NMR $\left(400 \mathrm{MHz}, \mathrm{CDCl}_{3}, 25^{\circ} \mathrm{C}, \mathrm{TMS}\right): \delta=7.75(\mathrm{dd}$, $\left.{ }^{3} J(\mathrm{H}, \mathrm{H})=8.1 \mathrm{~Hz}, \quad{ }^{4} J(\mathrm{H}, \mathrm{H})=1.1 \mathrm{~Hz}, \quad 1 \mathrm{H}\right), 7.40 \quad\left(\mathrm{ddd}, \quad{ }^{3} J(\mathrm{H}, \mathrm{H})=8.5 \mathrm{~Hz}, \quad{ }^{3} J(\mathrm{H}, \mathrm{H})=7.2 \mathrm{~Hz}\right.$, 
$\left.{ }^{4} J(\mathrm{H}, \mathrm{H})=1.7 \mathrm{~Hz}, 1 \mathrm{H}\right), 7.01\left(\mathrm{~d},{ }^{3} J(\mathrm{H}, \mathrm{H})=8.5 \mathrm{~Hz}, 1 \mathrm{H}\right), 7.04\left(\mathrm{br} \mathrm{d},{ }^{3} J(\mathrm{H}, \mathrm{H})=7.9 \mathrm{~Hz}, 1 \mathrm{H} ; \mathrm{NH}\right)$, $6.92\left(\mathrm{ddd},{ }^{3} J(\mathrm{H}, \mathrm{H})=8.1 \mathrm{~Hz},{ }^{3} J(\mathrm{H}, \mathrm{H})=7.2 \mathrm{~Hz},{ }^{4} J(\mathrm{H}, \mathrm{H})=1.0 \mathrm{~Hz}, 1 \mathrm{H}\right), 4.36-4.47(\mathrm{~m}, 2 \mathrm{H}), 3.72$ (s, 3H), 3.62-3.80 (m, 1H), 2.78-2.90 (m, 1H), 2.51-2.64 (m, 1H), 1.99-2.15 (m, 2H), 1.82$1.94(\mathrm{~m}, 1 \mathrm{H}), 1.52\left(\mathrm{ddd},{ }^{2} J(\mathrm{H}, \mathrm{H})=15 \mathrm{~Hz},{ }^{3} \mathrm{~J}(\mathrm{H}, \mathrm{H})=10 \mathrm{~Hz},{ }^{3} \mathrm{~J}(\mathrm{H}, \mathrm{H})=4.6 \mathrm{~Hz}, 1 \mathrm{H}\right), 1.38$ (ddd, $\left.{ }^{2} J(\mathrm{H}, \mathrm{H})=15 \mathrm{~Hz}, \quad{ }^{3} J(\mathrm{H}, \mathrm{H})=10 \mathrm{~Hz}, \quad{ }^{3} J(\mathrm{H}, \mathrm{H})=5.0 \mathrm{~Hz}, 1 \mathrm{H}\right), \quad 0.93-1.08(\mathrm{~m}, 1 \mathrm{H}), 0.63 \quad(\mathrm{~d}$, $\left.{ }^{3} J(\mathrm{H}, \mathrm{H})=6.6 \mathrm{~Hz}, 3 \mathrm{H}\right), 0.62 \mathrm{ppm}\left(\mathrm{d},{ }^{3} J(\mathrm{H}, \mathrm{H})=6.6 \mathrm{~Hz}, 3 \mathrm{H}\right)$; IR $\left(\mathrm{CCl}_{4}\right): 3383(\mathrm{NH}), 1751$, $1686(\mathrm{C}=\mathrm{O}) \mathrm{cm}^{-1} ; \mathrm{MS}(\mathrm{FAB}): \mathrm{m} / \mathrm{z} 386.2\left[\mathrm{M}+\mathrm{Na}^{+}\right]$.

(S)-4-NPP amide of L-leucine methyl ester ( $5, \mathrm{R}=(S)$-4-NPP)

$[\alpha]_{\mathrm{D}}^{25}=-295(c=1.0$ in $\mathrm{MeOH}) ;{ }^{1} \mathrm{H}$ NMR $\left(400 \mathrm{MHz}, \mathrm{CDCl}_{3}, 25^{\circ} \mathrm{C}, \mathrm{TMS}\right): \delta=8.16(\mathrm{~d}$, $\left.{ }^{3} J(\mathrm{H}, \mathrm{H})=9.1 \mathrm{~Hz} 2 \mathrm{H}\right), 6.63\left(\mathrm{~d},{ }^{3} J(\mathrm{H}, \mathrm{H})=9.1 \mathrm{~Hz} 2 \mathrm{H}\right), 6.38\left(\right.$ br d, $\left.{ }^{3} J(\mathrm{H}, \mathrm{H})=8.4 \mathrm{~Hz}, 1 \mathrm{H} ; \mathrm{NH}\right)$, 4.59-4.67 (m, $1 \mathrm{H}), \quad 4.19 \quad\left(\mathrm{dd},{ }^{3} J(\mathrm{H}, \mathrm{H})=7.7 \mathrm{~Hz},{ }^{3} J(\mathrm{H}, \mathrm{H})=3.6 \mathrm{~Hz}, 1 \mathrm{H}\right), 3.74 \quad(\mathrm{ddd}$, $\left.{ }^{2} J(\mathrm{H}, \mathrm{H})=9.5 \mathrm{~Hz},{ }^{3} J(\mathrm{H}, \mathrm{H})=7.6 \mathrm{~Hz},{ }^{3} J(\mathrm{H}, \mathrm{H})=1.7 \mathrm{~Hz}, 1 \mathrm{H}\right), 3.66(\mathrm{~s}, 3 \mathrm{H}), 3.37-3.46(\mathrm{~m}, 1 \mathrm{H})$, 2.30-2.38 (m, 2H), 2.11-2.23 (m, 1H), 1.97-2.08 (m, 1H), 1.46-1.69 (m, 3H), $0.933(\mathrm{~d}$, $\left.{ }^{3} J(\mathrm{H}, \mathrm{H})=6.3 \mathrm{~Hz}, 3 \mathrm{H}\right), 0.928 \mathrm{ppm}\left(\mathrm{d},{ }^{3} J(\mathrm{H}, \mathrm{H})=6.3 \mathrm{~Hz}, 3 \mathrm{H}\right) ; \mathrm{IR}\left(\mathrm{CCl}_{4}\right): 3411(\mathrm{NH}), 1747$, $1686(\mathrm{C}=\mathrm{O}) \mathrm{cm}^{-1} ; \mathrm{MS}(\mathrm{FAB}): \mathrm{m} / z 386.3\left[\mathrm{M}+\mathrm{Na}^{+}\right]$.

(R)-4-NPP amide of L-leucine methyl ester $(5, \mathrm{R}=(R)-4-\mathrm{NPP})$

$[\alpha]_{\mathrm{D}}{ }^{25}=+293(c=0.1$ in $\mathrm{MeOH}) ;{ }^{1} \mathrm{H}$ NMR $\left(300 \mathrm{MHz}, \mathrm{CDCl}_{3}, 25^{\circ} \mathrm{C}, \mathrm{TMS}\right): \delta=8.15(\mathrm{~d}$, $\left.{ }^{3} J(\mathrm{H}, \mathrm{H})=9.3 \mathrm{~Hz}, 2 \mathrm{H}\right), 6.60\left(\mathrm{~d},{ }^{3} J(\mathrm{H}, \mathrm{H})=9.3 \mathrm{~Hz}, 2 \mathrm{H}\right), 6.33\left(\mathrm{br} \mathrm{d},{ }^{3} J(\mathrm{H}, \mathrm{H})=8.5 \mathrm{~Hz}, 1 \mathrm{H} ; \mathrm{NH}\right)$, 4.61-4.68 (m, 1H), 4.19-4.23 (m, 1H), 3.75-3.83 (m, 1H), $3.73(\mathrm{~s}, 3 \mathrm{H}), 3.38-3.46(\mathrm{~m}, 1 \mathrm{H})$, 2.29-2.39 (m, 2H), 2.10-2.20 (m, 2H), 1.51-1.61 (m, 1H), 1.33-1.45 (m, 2H), $0.86(\mathrm{~d}$, $\left.{ }^{3} J(\mathrm{H}, \mathrm{H})=6.3 \mathrm{~Hz}, 3 \mathrm{H}\right), 0.80 \mathrm{ppm}\left(\mathrm{d},{ }^{3} J(\mathrm{H}, \mathrm{H})=6.3 \mathrm{~Hz}, 3 \mathrm{H}\right)$; IR $\left(\mathrm{CCl}_{4}\right): 3415(\mathrm{NH}), 1744$, $1687(\mathrm{C}=\mathrm{O}) \mathrm{cm}^{-1}$; MS(FAB): $\mathrm{m} / z 386.3\left[\mathrm{M}^{+} \mathrm{Na}^{+}\right]$.

(S)-2-NPP amide of L-tryptophan methyl ester $(6, \mathrm{R}=(S)$-2-NPP)

$[\alpha]_{\mathrm{D}}{ }^{25}=-790\left(c=1.0\right.$ in $\left.\mathrm{CHCl}_{3}\right) ;{ }^{1} \mathrm{H}$ NMR $\left(400 \mathrm{MHz}, \mathrm{CDCl}_{3}, 25^{\circ} \mathrm{C}, \mathrm{TMS}\right): \delta=8.11(\mathrm{br} \mathrm{s}, 1 \mathrm{H})$, $7.76\left(\mathrm{dd},{ }^{3} J(\mathrm{H}, \mathrm{H})=8.1 \mathrm{~Hz},{ }^{4} J(\mathrm{H}, \mathrm{H})=1.7 \mathrm{~Hz}, 1 \mathrm{H}\right), 7.58\left(\mathrm{~d},{ }^{3} J(\mathrm{H}, \mathrm{H})=8.0 \mathrm{~Hz}, 1 \mathrm{H}\right), 7.35-7.39$ (m, 2H), $7.19\left(\mathrm{ddd},{ }^{3} J(\mathrm{H}, \mathrm{H})=8.2 \mathrm{~Hz},{ }^{3} J(\mathrm{H}, \mathrm{H})=7.0 \mathrm{~Hz},{ }^{4} J(\mathrm{H}, \mathrm{H})=1.3 \mathrm{~Hz}, 1 \mathrm{H}\right), 7.13$ (ddd, $\left.{ }^{3} J(\mathrm{H}, \mathrm{H})=8.0 \mathrm{~Hz},{ }^{3} J(\mathrm{H}, \mathrm{H})=7.0 \mathrm{~Hz},{ }^{4} J(\mathrm{H}, \mathrm{H})=1.2 \mathrm{~Hz}, 1 \mathrm{H}\right), 7.06\left(\mathrm{~d},{ }^{3} J(\mathrm{H}, \mathrm{H})=2.4 \mathrm{~Hz}, 1 \mathrm{H}\right), 7.02$ (br d, $\left.{ }^{3} J(\mathrm{H}, \mathrm{H})=8.0 \mathrm{~Hz}, 1 \mathrm{H} ; \mathrm{CONH}\right), 6.93\left(\mathrm{dd},{ }^{3} J(\mathrm{H}, \mathrm{H})=8.5 \mathrm{~Hz},{ }^{3} J(\mathrm{H}, \mathrm{H})=1.0 \mathrm{~Hz}, 1 \mathrm{H}\right), 6.88$ (ddd, $\left.{ }^{3} J(\mathrm{H}, \mathrm{H})=8.1 \mathrm{~Hz},{ }^{3} J(\mathrm{H}, \mathrm{H})=7.2 \mathrm{~Hz},{ }^{4} J(\mathrm{H}, \mathrm{H})=1.0 \mathrm{~Hz}, 1 \mathrm{H}\right), 4.79-4.84(\mathrm{~m}, 1 \mathrm{H}), 4.28(\mathrm{dd}$, $\left.{ }^{3} J(\mathrm{H}, \mathrm{H})=9.0 \mathrm{~Hz},{ }^{3} J(\mathrm{H}, \mathrm{H})=7.0 \mathrm{~Hz}, 1 \mathrm{H}\right), 3.46(\mathrm{~s}, 3 \mathrm{H}), 3.36-3.43 \quad(\mathrm{~m}, 1 \mathrm{H}), 3.31(\mathrm{dd}$, 
$\left.{ }^{2} J(\mathrm{H}, \mathrm{H})=14.0 \mathrm{~Hz},{ }^{3} J(\mathrm{H}, \mathrm{H})=7.0 \mathrm{~Hz}, 1 \mathrm{H}\right), 3.18\left(\mathrm{dd},{ }^{2} J(\mathrm{H}, \mathrm{H})=14.0 \mathrm{~Hz},{ }^{3} J(\mathrm{H}, \mathrm{H})=7.9 \mathrm{~Hz}, 1 \mathrm{H}\right)$, 2.64-2.71 (m, 1H), 2.32-2.39 (m, 1H), 1.65-1.80 (m, 2H), 1.41-1.51 ppm (m, 1H); IR (CCl4): 3487, $3382(\mathrm{NH}), 1752,1682(\mathrm{C}=\mathrm{O}) \mathrm{cm}^{-1}$; MS(FAB): $m / z 459.3\left[\mathrm{M}+\mathrm{Na}^{+}\right]$.

(R)-2-NPP amide of L-tryptophan methyl ester $(6, \mathrm{R}=(R)-2-\mathrm{NPP})$

$[\alpha]_{\mathrm{D}}{ }^{25}=+793\left(c=1.0\right.$ in $\left.\mathrm{CHCl}_{3}\right) ;{ }^{1} \mathrm{H}$ NMR $\left(400 \mathrm{MHz}, \mathrm{CDCl}_{3}, 25^{\circ} \mathrm{C}, \mathrm{TMS}\right): \delta=7.70(\mathrm{dd}$, $\left.{ }^{3} J(\mathrm{H}, \mathrm{H})=8.1 \mathrm{~Hz},{ }^{4} J(\mathrm{H}, \mathrm{H})=1.7 \mathrm{~Hz}, 1 \mathrm{H}\right), 7.69$ (br s, $\left.1 \mathrm{H}\right), 7.35\left(\mathrm{~d},{ }^{3} J(\mathrm{H}, \mathrm{H})=8.0 \mathrm{~Hz}, 1 \mathrm{H}\right)$, $7.21\left(\mathrm{~d},{ }^{3} J(\mathrm{H}, \mathrm{H})=8.0 \mathrm{~Hz}, 1 \mathrm{H}\right), 7.19\left(\mathrm{ddd},{ }^{3} J(\mathrm{H}, \mathrm{H})=8.2 \mathrm{~Hz},{ }^{3} J(\mathrm{H}, \mathrm{H})=7.0 \mathrm{~Hz},{ }^{4} J(\mathrm{H}, \mathrm{H})=1.7 \mathrm{~Hz}\right.$, $1 \mathrm{H}), 7.11\left(\mathrm{ddd},{ }^{3} J(\mathrm{H}, \mathrm{H})=8.0 \mathrm{~Hz},{ }^{3} J(\mathrm{H}, \mathrm{H})=7.2 \mathrm{~Hz},{ }^{4} J(\mathrm{H}, \mathrm{H})=1.1 \mathrm{~Hz}, 1 \mathrm{H}\right), 7.00 \quad(\mathrm{br} \mathrm{d}$, $\left.{ }^{3} J(\mathrm{H}, \mathrm{H})=9.0 \mathrm{~Hz}, 1 \mathrm{H} ; \mathrm{CONH}\right), 6.98\left(\mathrm{ddd},{ }^{3} J(\mathrm{H}, \mathrm{H})=8.0 \mathrm{~Hz},{ }^{3} J(\mathrm{H}, \mathrm{H})=7.2 \mathrm{~Hz},{ }^{4} J(\mathrm{H}, \mathrm{H})=1.1 \mathrm{~Hz}\right.$, $1 \mathrm{H}), \quad 6.82\left(\mathrm{ddd}, \quad{ }^{3} J(\mathrm{H}, \mathrm{H})=8.1 \mathrm{~Hz},{ }^{3} J(\mathrm{H}, \mathrm{H})=7.0 \mathrm{~Hz},{ }^{4} J(\mathrm{H}, \mathrm{H})=1.1 \mathrm{~Hz}, \quad 1 \mathrm{H}\right), 6.73 \quad(\mathrm{~d}$, $\left.{ }^{3} J(\mathrm{H}, \mathrm{H})=8.2 \mathrm{~Hz}, 1 \mathrm{H}\right), 6.46 \quad\left(\mathrm{~d},{ }^{3} J(\mathrm{H}, \mathrm{H})=2.4 \mathrm{~Hz}, 1 \mathrm{H}\right), 4.79-4.84 \quad(\mathrm{~m}, 1 \mathrm{H}), 4.25 \quad(\mathrm{dd}$, $\left.{ }^{3} J(\mathrm{H}, \mathrm{H})=8.4 \mathrm{~Hz},{ }^{3} J(\mathrm{H}, \mathrm{H})=7.0 \mathrm{~Hz}, 1 \mathrm{H}\right), 3.70(\mathrm{~s}, 3 \mathrm{H}), 3.56-3.67 \quad(\mathrm{~m}, 1 \mathrm{H}), 3.16(\mathrm{dd}$, $\left.{ }^{2} J(\mathrm{H}, \mathrm{H})=14.0 \mathrm{~Hz},{ }^{3} J(\mathrm{H}, \mathrm{H})=7.0 \mathrm{~Hz}, 1 \mathrm{H}\right), 3.10\left(\mathrm{dd},{ }^{2} J(\mathrm{H}, \mathrm{H})=14 \mathrm{~Hz},{ }^{3} J(\mathrm{H}, \mathrm{H})=6.0 \mathrm{~Hz}, 1 \mathrm{H}\right)$, 2.68-2.77 (m, 1H), 2.46-2.56 (m, 1H), 1.96-2.08 (m, 2H), 1.74-1.86 ppm (m, 1H); IR (CCl4): $3487,3385(\mathrm{NH}), 1751,1684(\mathrm{C}=\mathrm{O}) \mathrm{cm}^{-1}$; MS(FAB): $m / z 459.3\left[\mathrm{M}+\mathrm{Na}^{+}\right]$.

(S)-4-NPP amide of L-tryptophan methyl ester (6, R = (S)-4-NPP)

$[\alpha]_{\mathrm{D}}{ }^{25}=-229(c=1.0$ in $\mathrm{MeOH}) ;{ }^{1} \mathrm{H}$ NMR $\left(400 \mathrm{MHz}, \mathrm{CDCl}_{3}, 25^{\circ} \mathrm{C}, \mathrm{TMS}\right): \delta=8.06$ (br s, $\left.1 \mathrm{H}\right)$, $8.00\left(\mathrm{~d},{ }^{3} J(\mathrm{H}, \mathrm{H})=8.6 \mathrm{~Hz}, 2 \mathrm{H}\right), 7.44\left(\mathrm{~d},{ }^{3} J(\mathrm{H}, \mathrm{H})=8.0 \mathrm{~Hz}, 1 \mathrm{H}\right), 7.33\left(\mathrm{ddd},{ }^{3} J(\mathrm{H}, \mathrm{H})=8.5 \mathrm{~Hz}\right.$, $\left.{ }^{4} J(\mathrm{H}, \mathrm{H})=1.0 \mathrm{~Hz}, \quad,{ }^{4} J(\mathrm{H}, \mathrm{H})=1.0 \mathrm{~Hz}, 1 \mathrm{H}\right), 7.21 \quad\left(\mathrm{ddd},{ }^{3} J(\mathrm{H}, \mathrm{H})=8.5 \mathrm{~Hz},{ }^{3} J(\mathrm{H}, \mathrm{H})=7.0 \mathrm{~Hz}\right.$, $\left.{ }^{4} J(\mathrm{H}, \mathrm{H})=1.0 \mathrm{~Hz}, 1 \mathrm{H}\right), 7.12\left(\mathrm{ddd},{ }^{3} J(\mathrm{H}, \mathrm{H})=8.0 \mathrm{~Hz},{ }^{3} J(\mathrm{H}, \mathrm{H})=7.0 \mathrm{~Hz},{ }^{4} J(\mathrm{H}, \mathrm{H})=1.0 \mathrm{~Hz}, 1 \mathrm{H}\right)$, $6.86\left(\mathrm{~d},{ }^{3} J(\mathrm{H}, \mathrm{H})=2.4 \mathrm{~Hz}, 1 \mathrm{H}\right), 6.33-6.37 \quad(\mathrm{~m}, 3 \mathrm{H}), 4.78-4.83 \quad(\mathrm{~m}, 1 \mathrm{H}), 4.06(\mathrm{dd}$, $\left.{ }^{3} J(\mathrm{H}, \mathrm{H})=8.0 \mathrm{~Hz}, \quad{ }^{3} J(\mathrm{H}, \mathrm{H})=3.0 \mathrm{~Hz}, \quad 1 \mathrm{H}\right), \quad 3.72 \quad(\mathrm{~s}, \quad 3 \mathrm{H}), \quad 3.33\left(\mathrm{dd}, \quad{ }^{2} J(\mathrm{H}, \mathrm{H})=14.0 \mathrm{~Hz}\right.$, $\left.{ }^{3} J(\mathrm{H}, \mathrm{H})=5.5 \mathrm{~Hz}, 1 \mathrm{H}\right), 3.25\left(\mathrm{dd},{ }^{2} J(\mathrm{H}, \mathrm{H})=14.0 \mathrm{~Hz},{ }^{3} J(\mathrm{H}, \mathrm{H})=6.0 \mathrm{~Hz}, 1 \mathrm{H}\right), 3.08-3.22(\mathrm{~m}, 2 \mathrm{H})$, 2.13-2.22 (m, 2H), 1.83-1.93 (m, 1H), 1.49-1.65 ppm (m, 1H); IR (CCl $\left.{ }_{4}\right): 3486,3398(\mathrm{NH})$, $1755,1686(\mathrm{C}=\mathrm{O}) \mathrm{cm}^{-1}$; MS(FAB): $m / z 459.1\left[\mathrm{M}+\mathrm{Na}^{+}\right]$.

(R)-4-NPP amide of L-tryptophan methyl ester $(6, \mathrm{R}=(R)-4-\mathrm{NPP})$

$[\alpha]_{\mathrm{D}}{ }^{25}=+216(c=1.0$ in $\mathrm{MeOH}) ;{ }^{1} \mathrm{H}$ NMR $\left(300 \mathrm{MHz}, \mathrm{CDCl}_{3}, 25^{\circ} \mathrm{C}, \mathrm{TMS}\right): \delta=7.96(\mathrm{~d}$, $\left.{ }^{3} J(\mathrm{H}, \mathrm{H})=9.4 \mathrm{~Hz}, 2 \mathrm{H}\right), 7.90($ br s, $1 \mathrm{H}), 7.31\left(\mathrm{dd},{ }^{3} J(\mathrm{H}, \mathrm{H})=8.5 \mathrm{~Hz},{ }^{4} J(\mathrm{H}, \mathrm{H})=1.0 \mathrm{~Hz}, 1 \mathrm{H}\right), 7.28$ $\left(\mathrm{dd},{ }^{3} J(\mathrm{H}, \mathrm{H})=8.5 \mathrm{~Hz},{ }^{4} J(\mathrm{H}, \mathrm{H})=1.0 \mathrm{~Hz}, 1 \mathrm{H}\right), 7.18\left(\mathrm{ddd},{ }^{3} J(\mathrm{H}, \mathrm{H})=8.5 \mathrm{~Hz},{ }^{3} J(\mathrm{H}, \mathrm{H})=7.0 \mathrm{~Hz}\right.$, $\left.{ }^{4} J(\mathrm{H}, \mathrm{H})=1.0 \mathrm{~Hz}, 1 \mathrm{H}\right), 7.00\left(\mathrm{ddd},{ }^{3} J(\mathrm{H}, \mathrm{H})=8.5 \mathrm{~Hz},{ }^{3} J(\mathrm{H}, \mathrm{H})=7.0 \mathrm{~Hz},{ }^{4} J(\mathrm{H}, \mathrm{H})=1.0 \mathrm{~Hz}, 1 \mathrm{H}\right)$, 
$6.57\left(\mathrm{~d},{ }^{3} J(\mathrm{H}, \mathrm{H})=2.4 \mathrm{~Hz}, 1 \mathrm{H}\right), 6.41 \quad\left(\right.$ br $\left.\mathrm{d},{ }^{3} J(\mathrm{H}, \mathrm{H})=7.7 \mathrm{~Hz}, 1 \mathrm{H} ; \mathrm{CONH}\right), 6.31 \quad(\mathrm{~d}$, $\left.{ }^{3} J(\mathrm{H}, \mathrm{H})=9.4 \mathrm{~Hz}, 2 \mathrm{H}\right), 4.83-4.88(\mathrm{~m}, 1 \mathrm{H}), 4.07\left(\mathrm{dd},{ }^{3} J(\mathrm{H}, \mathrm{H})=8.5 \mathrm{~Hz},{ }^{3} J(\mathrm{H}, \mathrm{H})=2.5 \mathrm{~Hz}, 1 \mathrm{H}\right)$, $3.75(\mathrm{~s}, 3 \mathrm{H}), 3.41-3.47(\mathrm{~m}, 1 \mathrm{H}), 3.14-3.30(\mathrm{~m}, 3 \mathrm{H}), 2.15-2.34(\mathrm{~m}, 2 \mathrm{H}), 1.92-2.08 \mathrm{ppm}(\mathrm{m}$, $2 \mathrm{H})$; IR (CCl 4$): 3644,3412(\mathrm{NH}), 1748,1685(\mathrm{C}=\mathrm{O}) \mathrm{cm}^{-1}$; MS(FAB): $m / z 437.3\left[\mathrm{M}+\mathrm{H}^{+}\right]$.

(S)-2-NPP amide of $L$-phenylalanine methyl ester (7, R = $(S)$-2-NPP)

$[\alpha]_{\mathrm{D}}^{25}=-917\left(c=1.0\right.$ in $\left.\mathrm{CHCl}_{3}\right) ;{ }^{1} \mathrm{H}$ NMR $\left(300 \mathrm{MHz}, \mathrm{CDCl}_{3}, 25^{\circ} \mathrm{C}, \mathrm{TMS}\right): \delta=7.77$ (dd, $\left.{ }^{3} J(\mathrm{H}, \mathrm{H})=8.1 \mathrm{~Hz}, \quad{ }^{4} J(\mathrm{H}, \mathrm{H})=1.7 \mathrm{~Hz}, \quad 1 \mathrm{H}\right), 7.40 \quad\left(\mathrm{ddd}, \quad{ }^{3} J(\mathrm{H}, \mathrm{H})=8.5 \mathrm{~Hz}, \quad{ }^{3} J(\mathrm{H}, \mathrm{H})=7.2 \mathrm{~Hz}\right.$, $\left.{ }^{4} J(\mathrm{H}, \mathrm{H})=1.7 \mathrm{~Hz}, \quad 1 \mathrm{H}\right), \quad 7.28-7.31 \quad(\mathrm{~m}, \quad 2 \mathrm{H}), 7.23 \quad\left(\mathrm{t},{ }^{3} J(\mathrm{H}, \mathrm{H})=7.4 \mathrm{~Hz}, 1 \mathrm{H}\right), \quad 7.17(\mathrm{~d}$, $\left.{ }^{3} J(\mathrm{H}, \mathrm{H})=7.7 \mathrm{~Hz}, 2 \mathrm{H}\right), 7.05\left(\mathrm{br} \mathrm{d},{ }^{3} J(\mathrm{H}, \mathrm{H})=8.5 \mathrm{~Hz}, 1 \mathrm{H} ; \mathrm{NH}\right), 6.96\left(\mathrm{dd},{ }^{3} J(\mathrm{H}, \mathrm{H})=8.5 \mathrm{~Hz}\right.$, $\left.{ }^{4} J(\mathrm{H}, \mathrm{H})=1.0 \mathrm{~Hz}, 1 \mathrm{H}\right), 6.91\left(\mathrm{ddd},{ }^{3} J(\mathrm{H}, \mathrm{H})=8.1 \mathrm{~Hz},{ }^{3} J(\mathrm{H}, \mathrm{H})=7.2 \mathrm{~Hz},{ }^{4} J(\mathrm{H}, \mathrm{H})=1.0 \mathrm{~Hz}, 1 \mathrm{H}\right)$, 4.71-4.76 (m, 1H), 4.31-4.35 (m, 1H), 3.48-3.56 (m, 1H), $3.52(\mathrm{~s}, 3 \mathrm{H}), 3.22$ (dd, $\left.{ }^{2} J(\mathrm{H}, \mathrm{H})=14.0 \mathrm{~Hz},{ }^{3} J(\mathrm{H}, \mathrm{H})=5.2 \mathrm{~Hz}, 1 \mathrm{H}\right), 2.90\left(\mathrm{dd},{ }^{2} J(\mathrm{H}, \mathrm{H})=14.0 \mathrm{~Hz},{ }^{3} J(\mathrm{H}, \mathrm{H})=8.9 \mathrm{~Hz}, 1 \mathrm{H}\right)$, 2.72-2.79 (m, 1H), 2.35-2.44 (m, 1H), 1.70-1.90 (m, 2H), 1.50-1.57 ppm (m, 1H); IR (CCl4): $3378(\mathrm{NH}), 1753,1684(\mathrm{C}=\mathrm{O}) \mathrm{cm}^{-1}$; $\mathrm{MS}(\mathrm{FAB}): m / z 420.2\left[\mathrm{M}+\mathrm{Na}^{+}\right]$.

(R)-2-NPP amide of L-phenylalanine methyl ester (7, $\mathrm{R}=(R)-2-\mathrm{NPP})$

$[\alpha]_{\mathrm{D}}{ }^{25}=+315\left(c=1.0\right.$ in $\left.\mathrm{CHCl}_{3}\right) ;{ }^{1} \mathrm{H}$ NMR $\left(300 \mathrm{MHz}, \mathrm{CDCl}_{3}, 25^{\circ} \mathrm{C}, \mathrm{TMS}\right): \delta=7.78(\mathrm{dd}$, $\left.{ }^{3} J(\mathrm{H}, \mathrm{H})=8.1 \mathrm{~Hz}, \quad{ }^{4} J(\mathrm{H}, \mathrm{H})=1.7 \mathrm{~Hz}, \quad 1 \mathrm{H}\right), 7.30 \quad\left(\mathrm{ddd}, \quad{ }^{3} J(\mathrm{H}, \mathrm{H})=8.5 \mathrm{~Hz}, \quad{ }^{3} J(\mathrm{H}, \mathrm{H})=7.2 \mathrm{~Hz}\right.$, $\left.{ }^{4} J(\mathrm{H}, \mathrm{H})=1.7 \mathrm{~Hz}, 1 \mathrm{H}\right), 6.97-7.02(\mathrm{~m}, 2 \mathrm{H}), 6.88-6.94(\mathrm{~m}, 3 \mathrm{H}), 6.81\left(\mathrm{dd},{ }^{3} J(\mathrm{H}, \mathrm{H})=8.5 \mathrm{~Hz}\right.$, $\left.{ }^{4} J(\mathrm{H}, \mathrm{H})=1.0 \mathrm{~Hz}, 1 \mathrm{H}\right), 6.71\left(\mathrm{~d},{ }^{3} J(\mathrm{H}, \mathrm{H})=8.1 \mathrm{~Hz}, 2 \mathrm{H}\right)$, , 4.72-4.79 (m, 1H), 4.29-4.34 (m, 1H), $3.75(\mathrm{~s}, 3 \mathrm{H}), 3.62-3.71(\mathrm{~m}, 1 \mathrm{H}), 3.03\left(\mathrm{dd},{ }^{2} J(\mathrm{H}, \mathrm{H})=14.0 \mathrm{~Hz},{ }^{3} J(\mathrm{H}, \mathrm{H})=5.0 \mathrm{~Hz}, 1 \mathrm{H}\right), 2.85(\mathrm{dd}$, $\left.{ }^{2} J(\mathrm{H}, \mathrm{H})=14.0 \mathrm{~Hz},{ }^{3} J(\mathrm{H}, \mathrm{H})=8.5 \mathrm{~Hz}, 1 \mathrm{H}\right), 2.72-2.79(\mathrm{~m}, 1 \mathrm{H}), 2.47-2.58(\mathrm{~m}, 1 \mathrm{H}), 1.94-2.09$ $(\mathrm{m}, 2 \mathrm{H}), 1.76-1.90 \mathrm{ppm}(\mathrm{m}, 1 \mathrm{H})$; IR $\left(\mathrm{CCl}_{4}\right): 3381(\mathrm{NH}), 1752,1685(\mathrm{C}=\mathrm{O}) \mathrm{cm}^{-1}$; $\mathrm{MS}(\mathrm{FAB}): m / z 420.1\left[\mathrm{M}+\mathrm{Na}^{+}\right]$.

\section{(S)-4-NPP amide of L-phenylalanine methyl ester (7, $\mathrm{R}=(S)$-4-NPP)}

$[\alpha]_{\mathrm{D}}{ }^{25}=-247(c=1.0$ in $\mathrm{MeOH}) ;{ }^{1} \mathrm{H}$ NMR $\left(300 \mathrm{MHz}, \mathrm{CDCl}_{3}, 25^{\circ} \mathrm{C}, \mathrm{TMS}\right): \delta=8.10(\mathrm{~d}$, $\left.{ }^{3} J(\mathrm{H}, \mathrm{H})=9.1 \mathrm{~Hz}, 2 \mathrm{H}\right), 7.22-7.29(\mathrm{~m}, 3 \mathrm{H}), 7.03-7.07(\mathrm{~m}, 2 \mathrm{H}), 6.52\left(\mathrm{~d},{ }^{3} J(\mathrm{H}, \mathrm{H})=9.1 \mathrm{~Hz}, 2 \mathrm{H}\right)$, $6.33\left(\mathrm{br} d,{ }^{3} J(\mathrm{H}, \mathrm{H})=7.8 \mathrm{~Hz}, 1 \mathrm{H} ; \mathrm{NH}\right), 4.80-4.87(\mathrm{~m}, 1 \mathrm{H}), 4.11\left(\mathrm{dd},{ }^{3} J(\mathrm{H}, \mathrm{H})=8.7 \mathrm{~Hz}\right.$, $\left.{ }^{3} J(\mathrm{H}, \mathrm{H})=2.4 \mathrm{~Hz}, 1 \mathrm{H}\right), 3.70(\mathrm{~s}, 3 \mathrm{H}), 3.51-3.55(\mathrm{~m}, 1 \mathrm{H}), 3.21-3.35(\mathrm{~m}, 2 \mathrm{H}), 3.01$ (dd, $\left.{ }^{2} J(\mathrm{H}, \mathrm{H})=14.0 \mathrm{~Hz},{ }^{3} J(\mathrm{H}, \mathrm{H})=7.2 \mathrm{~Hz}, 1 \mathrm{H}\right), 2.09-2.31(\mathrm{~m}, 2 \mathrm{H}), 1.93-2.06(\mathrm{~m}, 1 \mathrm{H}), 1.59-1.77$ ppm (m, 1H); IR ( $\left.\mathrm{CCl}_{4}\right)$ : $3410(\mathrm{NH}), 1747,1687(\mathrm{C}=\mathrm{O}) \mathrm{cm}^{-1}$; $\mathrm{MS}(\mathrm{FAB}): \mathrm{m} / z 420.3$ 
$\left[\mathrm{M}+\mathrm{Na}^{+}\right]$.

(R)-4-NPP amide of L-phenylalanine methyl ester (7, R = $(R)-4-\mathrm{NPP})$

$[\alpha]_{\mathrm{D}}{ }^{25}=+172\left(c=1.0\right.$ in $\left.\mathrm{CHCl}_{3}\right) ;{ }^{1} \mathrm{H}$ NMR $\left(300 \mathrm{MHz}, \mathrm{CDCl}_{3}, 25^{\circ} \mathrm{C}, \mathrm{TMS}\right): \delta=8.10$ (d, $\left.{ }^{3} J(\mathrm{H}, \mathrm{H})=9.5 \mathrm{~Hz}, 2 \mathrm{H}\right), 7.15 \quad\left(\mathrm{t},{ }^{3} J(\mathrm{H}, \mathrm{H})=7.3 \mathrm{~Hz}, 1 \mathrm{H}\right), 7.03-7.08 \quad(\mathrm{~m}, 2 \mathrm{H}), 6.78 \quad(\mathrm{~d}$, $\left.{ }^{3} J(\mathrm{H}, \mathrm{H})=7.3 \mathrm{~Hz}, 2 \mathrm{H}\right), 6.45\left(\mathrm{~d},{ }^{3} J(\mathrm{H}, \mathrm{H})=9.5 \mathrm{~Hz}, 2 \mathrm{H}\right), 6.32\left(\mathrm{br} \mathrm{d},{ }^{3} J(\mathrm{H}, \mathrm{H})=8.5 \mathrm{~Hz}, 1 \mathrm{H} ; \mathrm{NH}\right)$, 4.85-4.93 (m, 1H), 4.11-4.17 (m, 1H), $3.76(\mathrm{~s}, 3 \mathrm{H}), 3.56-3.65(\mathrm{~m}, 1 \mathrm{H}), 3.29-3.38(\mathrm{~m}, 1 \mathrm{H})$, $3.06\left(\mathrm{dd}, \quad{ }^{2} J(\mathrm{H}, \mathrm{H})=14.0 \mathrm{~Hz}, \quad{ }^{3} J(\mathrm{H}, \mathrm{H})=5.4 \mathrm{~Hz} \quad, 1 \mathrm{H}\right), \quad 2.97 \quad\left(\mathrm{dd}, \quad{ }^{2} J(\mathrm{H}, \mathrm{H})=14.0 \mathrm{~Hz}\right.$, $\left.{ }^{3} J(\mathrm{H}, \mathrm{H})=7.2 \mathrm{~Hz}, 1 \mathrm{H}\right), 2.22-2.31(\mathrm{~m}, 2 \mathrm{H}), 2.00-2.11 \mathrm{ppm}(\mathrm{m}, 2 \mathrm{H})$; IR $\left(\mathrm{CCl}_{4}\right): 3410(\mathrm{NH})$, 1747, $1687(\mathrm{C}=\mathrm{O}) \mathrm{cm}^{-1}$; MS(FAB): $m / z 420.3\left[\mathrm{M}+\mathrm{Na}^{+}\right]$.

(S)-2-NPP amide of (1R)-(+)-bornylamine $(8, \mathrm{R}=(S)$-2-NPP)

$[\alpha]_{\mathrm{D}}{ }^{25}=-876\left(c=1.0\right.$ in $\left.\mathrm{CHCl}_{3}\right) ;{ }^{1} \mathrm{H}$ NMR $\left(400 \mathrm{MHz}, \mathrm{CDCl}_{3}, 25^{\circ} \mathrm{C}, \mathrm{TMS}\right): \delta=7.78(\mathrm{dd}$, $\left.{ }^{3} J(\mathrm{H}, \mathrm{H})=8.2 \mathrm{~Hz}, \quad{ }^{4} J(\mathrm{H}, \mathrm{H})=1.7 \mathrm{~Hz}, \quad 1 \mathrm{H}\right), 7.41 \quad\left(\mathrm{ddd}, \quad{ }^{3} J(\mathrm{H}, \mathrm{H})=8.5 \mathrm{~Hz}, \quad{ }^{3} J(\mathrm{H}, \mathrm{H})=7.4 \mathrm{~Hz}\right.$, $\left.{ }^{4} J(\mathrm{H}, \mathrm{H})=1.7 \mathrm{~Hz}, \quad 1 \mathrm{H}\right), \quad 7.04 \quad\left(\mathrm{~d},{ }^{3} J(\mathrm{H}, \mathrm{H})=8.5 \mathrm{~Hz}, \quad 1 \mathrm{H}\right), \quad 6.91 \quad\left(\mathrm{ddd}, \quad{ }^{3} J(\mathrm{H}, \mathrm{H})=8.2 \mathrm{~Hz}\right.$, $\left.{ }^{3} J(\mathrm{H}, \mathrm{H})=7.4 \mathrm{~Hz},{ }^{4} J(\mathrm{H}, \mathrm{H})=1.2 \mathrm{~Hz}, 1 \mathrm{H}\right), 6.79\left(\right.$ br d, $\left.{ }^{3} J(\mathrm{H}, \mathrm{H})=9.6 \mathrm{~Hz}, 1 \mathrm{H} ; \mathrm{NH}\right), 4.42(\mathrm{dd}$, $\left.{ }^{3} J(\mathrm{H}, \mathrm{H})=8.9 \mathrm{~Hz},{ }^{3} J(\mathrm{H}, \mathrm{H})=6.9 \mathrm{~Hz}, 1 \mathrm{H}\right), 4.10-4.17(\mathrm{~m}, 1 \mathrm{H}), 3.65-3.72(\mathrm{~m}, 1 \mathrm{H}), 2.80-2.85(\mathrm{~m}$, $1 \mathrm{H}), 2.59-2.65(\mathrm{~m}, 1 \mathrm{H}), 2.24-2.33(\mathrm{~m}, 1 \mathrm{H}), 2.04-2.10(\mathrm{~m}, 1 \mathrm{H}), 1.86-2.01(\mathrm{~m}, 2 \mathrm{H}), 1.55-$ $1.61(\mathrm{~m}, 2 \mathrm{H}), 1.02-1.08(\mathrm{~m}, 1 \mathrm{H}), 0.88-0.93(\mathrm{~m}, 1 \mathrm{H}), 0.88(\mathrm{~s}, 3 \mathrm{H}), 0.77-0.81(\mathrm{~m}, 2 \mathrm{H}), 0.75$ (s, 3H), 0.48 ppm (s, 3H); IR $\left(\mathrm{CCl}_{4}\right): 3391(\mathrm{NH}), 1677(\mathrm{C}=\mathrm{O}) \mathrm{cm}^{-1}$; MS(FAB): $\mathrm{m} / z 394.3$ $\left[\mathrm{M}+\mathrm{Na}^{+}\right]$.

(R)-2-NPP amide of (1R)-(+)-bornylamine $(8, \mathrm{R}=(R)-2-\mathrm{NPP})$

$[\alpha]_{\mathrm{D}}{ }^{25}=+880\left(c=1.0\right.$ in $\left.\mathrm{CHCl}_{3}\right) ;{ }^{1} \mathrm{H}$ NMR $\left(400 \mathrm{MHz}, \mathrm{CDCl}_{3}, 25^{\circ} \mathrm{C}, \mathrm{TMS}\right): \delta=7.78(\mathrm{dd}$, $\left.{ }^{3} J(\mathrm{H}, \mathrm{H})=8.2 \mathrm{~Hz}, \quad{ }^{4} J(\mathrm{H}, \mathrm{H})=1.7 \mathrm{~Hz}, \quad 1 \mathrm{H}\right), 7.40 \quad\left(\mathrm{ddd}, \quad{ }^{3} J(\mathrm{H}, \mathrm{H})=8.5 \mathrm{~Hz}, \quad{ }^{3} J(\mathrm{H}, \mathrm{H})=7.3 \mathrm{~Hz}\right.$, $\left.{ }^{4} J(\mathrm{H}, \mathrm{H})=1.7 \mathrm{~Hz}, \quad 1 \mathrm{H}\right), \quad 7.04 \quad\left(\mathrm{~d},{ }^{3} J(\mathrm{H}, \mathrm{H})=8.5 \mathrm{~Hz}, \quad 1 \mathrm{H}\right), \quad 6.92 \quad\left(\mathrm{ddd}, \quad{ }^{3} J(\mathrm{H}, \mathrm{H})=8.2 \mathrm{~Hz}\right.$, $\left.{ }^{3} J(\mathrm{H}, \mathrm{H})=7.3 \mathrm{~Hz},{ }^{4} J(\mathrm{H}, \mathrm{H})=1.1 \mathrm{~Hz}, 1 \mathrm{H}\right), 6.89\left(\mathrm{br} \mathrm{d},{ }^{3} J(\mathrm{H}, \mathrm{H})=9.6 \mathrm{~Hz}, 1 \mathrm{H} ; \mathrm{NH}\right), 4.41-4.45(\mathrm{~m}$, $1 \mathrm{H}), 4.06-4.13(\mathrm{~m}, 1 \mathrm{H}), 3.66-3.72(\mathrm{~m}, 1 \mathrm{H}), 2.82-2.87(\mathrm{~m}, 1 \mathrm{H}), 2.59-2.65(\mathrm{~m}, 1 \mathrm{H}), 2.03-$ $2.17(\mathrm{~m}, 2 \mathrm{H}), 1.84-2.01(\mathrm{~m}, 2 \mathrm{H}), 1.62-1.71(\mathrm{~m}, 1 \mathrm{H}), 1.52-1.54(\mathrm{~m}, 1 \mathrm{H}), 1.44-1.50(\mathrm{~m}, 1 \mathrm{H})$, 1.30-1.38 (m, 1H), 0.95-1.01 (m, 1H), $0.89(\mathrm{~s}, 3 \mathrm{H}), 0.84(\mathrm{~s}, 3 \mathrm{H}), 0.80$ (s, 3H), $0.39 \mathrm{ppm}(\mathrm{dd}$, $\left.{ }^{2} J(\mathrm{H}, \mathrm{H})=13.2 \mathrm{~Hz},{ }^{3} J(\mathrm{H}, \mathrm{H})=4.3 \mathrm{~Hz}, 1 \mathrm{H}\right)$; IR $\left(\mathrm{CCl}_{4}\right): 3386(\mathrm{NH}), 1678(\mathrm{C}=\mathrm{O}) \mathrm{cm}^{-1}$; $\mathrm{MS}(\mathrm{FAB}): m / z 394.4\left[\mathrm{M}+\mathrm{Na}^{+}\right]$. 
(S)-4-NPP amide of (1R)-(+)-bornylamine (8, R = (S)-4-NPP)

$[\alpha]_{\mathrm{D}}{ }^{25}=-200\left(c=1.0\right.$ in $\left.\mathrm{CHCl}_{3}\right) ;{ }^{1} \mathrm{H}$ NMR $\left(400 \mathrm{MHz}, \mathrm{CDCl}_{3}, 25^{\circ} \mathrm{C}, \mathrm{TMS}\right): \delta=8.17$ (d, $\left.{ }^{3} J(\mathrm{H}, \mathrm{H})=9.3 \mathrm{~Hz}, 2 \mathrm{H}\right), 6.62\left(\mathrm{~d},{ }^{3} J(\mathrm{H}, \mathrm{H})=9.3 \mathrm{~Hz}, 2 \mathrm{H}\right), 6.05\left(\mathrm{br} \mathrm{d},{ }^{3} J(\mathrm{H}, \mathrm{H})=9.5 \mathrm{~Hz}, 1 \mathrm{H} ; \mathrm{NH}\right)$, 4.20-4.26 (m, 2H), 3.74-3.79 (m, 1H), 3.41-3.47 (m, 1H), 2.31-2.38 (m, 3H), 2.11-2.19 (m, 1H), 1.99-2.09 (m, 1H), 1.61-1.70 (m, 2H), 1.11-1.18 (m, 1H), 0.95-1.01 (m, 1H), $0.92(\mathrm{~s}$, $3 \mathrm{H}), 0.81-0.87(\mathrm{~m}, 1 \mathrm{H}), 0.81(\mathrm{~s}, 3 \mathrm{H}), 0.71(\mathrm{~s}, 3 \mathrm{H}), 0.66 \mathrm{ppm}\left(\mathrm{dd},{ }^{2} J(\mathrm{H}, \mathrm{H})=13.6 \mathrm{~Hz}\right.$, $\left.{ }^{3} J(\mathrm{H}, \mathrm{H})=4.6 \mathrm{~Hz}, 1 \mathrm{H}\right)$; IR $\left(\mathrm{CCl}_{4}\right): 3420(\mathrm{NH}), 1679(\mathrm{C}=\mathrm{O}) \mathrm{cm}^{-1}$; $\mathrm{MS}(\mathrm{FAB}): \mathrm{m} / z 394.4$ $\left[\mathrm{M}+\mathrm{Na}^{+}\right]$.

( $R$ )-4-NPP amide of $(\mathbf{1})$-(+)-bornylamine $(8, \mathrm{R}=(R)$-4-NPP)

$[\alpha]_{\mathrm{D}}{ }^{25}=+199\left(c=0.1\right.$ in $\left.\mathrm{CHCl}_{3}\right) ;{ }^{1} \mathrm{H}$ NMR $\left(400 \mathrm{MHz}, \mathrm{CDCl}_{3}, 25^{\circ} \mathrm{C}, \mathrm{TMS}\right): \delta=8.17$ (d, $\left.{ }^{3} J(\mathrm{H}, \mathrm{H})=9.3 \mathrm{~Hz}, 2 \mathrm{H}\right), 6.76\left(\mathrm{~d},{ }^{3} J(\mathrm{H}, \mathrm{H})=9.3 \mathrm{~Hz}, 2 \mathrm{H}\right), 6.01\left(\mathrm{br} \mathrm{d},{ }^{3} J(\mathrm{H}, \mathrm{H})=9.3 \mathrm{~Hz}, 1 \mathrm{H} ; \mathrm{NH}\right)$, 4.18-4.24 (m, 2H), 3.72-3.77 (m, 1H), 3.41-3.46 (m, 1H), 2.25-2.37 (m, 3H), 2.14-2.20 (m, $1 \mathrm{H}), 2.02-2.12(\mathrm{~m}, 1 \mathrm{H}), 1.64-1.72(\mathrm{~m}, 1 \mathrm{H}), 1.60-1.62(\mathrm{~m}, 1 \mathrm{H}), 1.30-1.38(\mathrm{~m}, 1 \mathrm{H}), 1.12$ $\left(\mathrm{ddd},{ }^{2} J(\mathrm{H}, \mathrm{H})=13.7 \mathrm{~Hz},{ }^{3} J(\mathrm{H}, \mathrm{H})=9.4 \mathrm{~Hz},{ }^{3} J(\mathrm{H}, \mathrm{H})=4.3 \mathrm{~Hz}, 1 \mathrm{H}\right), 0.93(\mathrm{~s}, 3 \mathrm{H}), 0.87-0.93(\mathrm{~m}$, $1 \mathrm{H}), 0.84$ (s, 3H), 0.78 (s, 3H), $0.54 \mathrm{ppm}\left(\mathrm{dd},{ }^{2} J(\mathrm{H}, \mathrm{H})=13.4 \mathrm{~Hz},{ }^{3} J(\mathrm{H}, \mathrm{H})=4.3 \mathrm{~Hz}, 1 \mathrm{H}\right)$; IR $\left(\mathrm{CCl}_{4}\right): 3419(\mathrm{NH}), 1680(\mathrm{C}=\mathrm{O}) \mathrm{cm}^{-1}$; $\mathrm{MS}(\mathrm{FAB}): \mathrm{m} / z 394.3\left[\mathrm{M}+\mathrm{Na}^{+}\right]$.

(S)-2-NPP amide of L-leucinol $(9, \mathrm{R}=(S)$-2-NPP)

$[\alpha]_{\mathrm{D}}{ }^{25}=-598\left(c=1.0\right.$ in $\left.\mathrm{CHCl}_{3}\right) ;{ }^{1} \mathrm{H}$ NMR $\left(300 \mathrm{MHz}, \mathrm{CDCl}_{3}, 25^{\circ} \mathrm{C}, \mathrm{TMS}\right): \delta=7.77(\mathrm{dd}$, $\left.{ }^{3} J(\mathrm{H}, \mathrm{H})=8.1 \mathrm{~Hz}, \quad{ }^{4} J(\mathrm{H}, \mathrm{H})=1.7 \mathrm{~Hz}, \quad 1 \mathrm{H}\right), 7.43 \quad\left(\mathrm{ddd}, \quad{ }^{3} J(\mathrm{H}, \mathrm{H})=8.5 \mathrm{~Hz}, \quad{ }^{3} J(\mathrm{H}, \mathrm{H})=7.2 \mathrm{~Hz}\right.$, $\left.{ }^{4} J(\mathrm{H}, \mathrm{H})=1.7 \mathrm{~Hz}, \quad 1 \mathrm{H}\right), \quad 7.05 \quad\left(\mathrm{~d},{ }^{3} J(\mathrm{H}, \mathrm{H})=8.5 \mathrm{~Hz}, \quad 1 \mathrm{H}\right), 6.95 \quad\left(\mathrm{ddd}, \quad{ }^{3} J(\mathrm{H}, \mathrm{H})=8.1 \mathrm{~Hz}\right.$, $\left.{ }^{3} J(\mathrm{H}, \mathrm{H})=7.2 \mathrm{~Hz},{ }^{4} J(\mathrm{H}, \mathrm{H})=1.0 \mathrm{~Hz}, 1 \mathrm{H}\right), 6.85\left(\mathrm{br} \mathrm{d},{ }^{3} J(\mathrm{H}, \mathrm{H})=7.3 \mathrm{~Hz}, 1 \mathrm{H} ; \mathrm{NH}\right), 4.41-4.46(\mathrm{~m}$, $1 \mathrm{H}), 3.83-3.97(\mathrm{~m}, 1 \mathrm{H}), 3.58-3.71(\mathrm{~m}, 1 \mathrm{H}), 3.46-3.50(\mathrm{~m}, 1 \mathrm{H}), 3.27-3.31(\mathrm{~m}, 1 \mathrm{H}), 2.81-$ $2.88(\mathrm{~m}, 1 \mathrm{H}), 2.51-2.66(\mathrm{~m}, 1 \mathrm{H}), 2.35$ (br s, $1 \mathrm{H}), 1.84-2.08(\mathrm{~m}, 3 \mathrm{H}), 1.47-1.64(\mathrm{~m}, 1 \mathrm{H})$, 1.21-1.40 (m, 2H), $0.91\left(\mathrm{~d},{ }^{3} J(\mathrm{H}, \mathrm{H})=6.5 \mathrm{~Hz}, 3 \mathrm{H}\right), 0.88 \mathrm{ppm}\left(\mathrm{d},{ }^{3} J(\mathrm{H}, \mathrm{H})=6.5 \mathrm{~Hz}, 3 \mathrm{H}\right) ; \mathrm{IR}$ $\left(\mathrm{CCl}_{4}\right): 3372(\mathrm{NH}), 1676(\mathrm{C}=\mathrm{O}) \mathrm{cm}^{-1}$; $\mathrm{MS}(\mathrm{FAB}): \mathrm{m} / z 358.3\left[\mathrm{M}+\mathrm{Na}^{+}\right]$.

( $R$ )-2-NPP amide of L-leucinol $(9, \mathrm{R}=(R)$-2-NPP)

$[\alpha]_{\mathrm{D}}{ }^{25}=+700\left(c=1.0\right.$ in $\left.\mathrm{CHCl}_{3}\right) ;{ }^{1} \mathrm{H}$ NMR $\left(300 \mathrm{MHz}, \mathrm{CDCl}_{3}, 25^{\circ} \mathrm{C}, \mathrm{TMS}\right): \delta=7.75(\mathrm{dd}$, $\left.{ }^{3} J(\mathrm{H}, \mathrm{H})=8.1 \mathrm{~Hz}, \quad{ }^{4} J(\mathrm{H}, \mathrm{H})=1.7 \mathrm{~Hz}, \quad 1 \mathrm{H}\right), 7.41 \quad\left(\mathrm{ddd}, \quad{ }^{3} J(\mathrm{H}, \mathrm{H})=8.5 \mathrm{~Hz}, \quad{ }^{3} J(\mathrm{H}, \mathrm{H})=7.2 \mathrm{~Hz}\right.$, $\left.{ }^{4} J(\mathrm{H}, \mathrm{H})=1.7 \mathrm{~Hz}, \quad 1 \mathrm{H}\right), 7.02 \quad\left(\mathrm{~d},{ }^{3} J(\mathrm{H}, \mathrm{H})=8.5 \mathrm{~Hz}, \quad 1 \mathrm{H}\right), 6.93 \quad\left(\mathrm{ddd},{ }^{3} J(\mathrm{H}, \mathrm{H})=8.1 \mathrm{~Hz}\right.$, $\left.{ }^{3} J(\mathrm{H}, \mathrm{H})=7.2 \mathrm{~Hz},{ }^{4} J(\mathrm{H}, \mathrm{H})=1.0 \mathrm{~Hz}, 1 \mathrm{H}\right), 6.74\left(\mathrm{br} \mathrm{d},{ }^{3} J(\mathrm{H}, \mathrm{H})=6.8 \mathrm{~Hz}, 1 \mathrm{H} ; \mathrm{NH}\right), 4.41-4.45(\mathrm{~m}$, 
1H), 3.79-3.93 (m, 1H), 3.57-3.72 (m, 2H), 3.43-3.52 (m, 1H), 2.82-2.87 (m, 1H), 2.73 (t, $\left.{ }^{3} J(\mathrm{H}, \mathrm{H})=5.2 \mathrm{~Hz}, 1 \mathrm{H}\right), 2.55-2.66(\mathrm{~m}, 1 \mathrm{H}), 1.83-2.11(\mathrm{~m}, 3 \mathrm{H}), 1.08-1.22(\mathrm{~m}, 2 \mathrm{H}), 0.81-0.94$ $(\mathrm{m}, 1 \mathrm{H}), 0.59\left(\mathrm{~d},{ }^{3} J(\mathrm{H}, \mathrm{H})=6.5 \mathrm{~Hz}, 3 \mathrm{H}\right), 0.58 \mathrm{ppm}\left(\mathrm{d},{ }^{3} J(\mathrm{H}, \mathrm{H})=6.5 \mathrm{~Hz}, 3 \mathrm{H}\right)$; IR $\left(\mathrm{CCl}_{4}\right): 3378$ $(\mathrm{NH}), 1669(\mathrm{C}=\mathrm{O}) \mathrm{cm}^{-1}$; $\mathrm{MS}(\mathrm{FAB}): m / z 358.3\left[\mathrm{M}+\mathrm{Na}^{+}\right]$.

(S)-2-NPP amide of L-phenylalaninol $(10, \mathrm{R}=(S)$-2-NPP)

$[\alpha]_{\mathrm{D}}^{25}=-1090\left(c=1.0\right.$ in $\left.\mathrm{CHCl}_{3}\right) ;{ }^{1} \mathrm{H}$ NMR $\left(300 \mathrm{MHz}, \mathrm{CDCl}_{3}, 25^{\circ} \mathrm{C}, \mathrm{TMS}\right): \delta=7.77(\mathrm{dd}$, $\left.{ }^{3} J(\mathrm{H}, \mathrm{H})=8.1 \mathrm{~Hz}, \quad{ }^{4} J(\mathrm{H}, \mathrm{H})=1.7 \mathrm{~Hz}, \quad 1 \mathrm{H}\right), 7.41 \quad\left(\mathrm{ddd}, \quad{ }^{3} J(\mathrm{H}, \mathrm{H})=8.5 \mathrm{~Hz}, \quad{ }^{3} J(\mathrm{H}, \mathrm{H})=7.2 \mathrm{~Hz}\right.$, $\left.{ }^{4} J(\mathrm{H}, \mathrm{H})=1.7 \mathrm{~Hz}, 1 \mathrm{H}\right), 7.26-7.30(\mathrm{~m}, 2 \mathrm{H}), 7.21\left(\mathrm{~d},{ }^{3} J(\mathrm{H}, \mathrm{H})=7.2 \mathrm{~Hz}, 2 \mathrm{H}\right), 7.19-7.21(\mathrm{~m}, 1 \mathrm{H})$, $6.98\left(\mathrm{dd}, \quad{ }^{3} J(\mathrm{H}, \mathrm{H})=8.5 \mathrm{~Hz}, \quad{ }^{3} J(\mathrm{H}, \mathrm{H})=1.0 \mathrm{~Hz}, \quad 1 \mathrm{H}\right), \quad 6.93 \quad\left(\mathrm{ddd}, \quad{ }^{3} J(\mathrm{H}, \mathrm{H})=8.1 \mathrm{~Hz}\right.$, $\left.{ }^{3} J(\mathrm{H}, \mathrm{H})=7.2 \mathrm{~Hz},{ }^{4} J(\mathrm{H}, \mathrm{H})=1.0 \mathrm{~Hz}, 1 \mathrm{H}\right), 6.87\left(\mathrm{br} \mathrm{d},{ }^{3} J(\mathrm{H}, \mathrm{H})=7.5 \mathrm{~Hz}, 1 \mathrm{H} ; \mathrm{NH}\right), 4.29-4.34(\mathrm{~m}$, $1 \mathrm{H})$, 4.04-4.17 (m, 1H), 3.37-3.59 (m, 3H), $2.92\left(\mathrm{dd},{ }^{2} J(\mathrm{H}, \mathrm{H})=14.0 \mathrm{~Hz},{ }^{3} J(\mathrm{H}, \mathrm{H})=6.2 \mathrm{~Hz}\right.$, $1 \mathrm{H}), 2.71-2.79(\mathrm{~m}, 1 \mathrm{H}), 2.66\left(\mathrm{dd},{ }^{2} J(\mathrm{H}, \mathrm{H})=14.0 \mathrm{~Hz},{ }^{3} J(\mathrm{H}, \mathrm{H})=9.5 \mathrm{~Hz}, 1 \mathrm{H}\right), 2.32-2.41(\mathrm{~m}$, $1 \mathrm{H}), 2.20\left(\mathrm{t},{ }^{3} \mathrm{~J}(\mathrm{H}, \mathrm{H})=5.8 \mathrm{~Hz}, 1 \mathrm{H}\right), 1.71-1.82(\mathrm{~m}, 2 \mathrm{H}), 1.41-1.51 \mathrm{ppm}(\mathrm{m}, 1 \mathrm{H})$; IR $\left(\mathrm{CCl}_{4}\right)$ : $3375(\mathrm{NH}), 1678(\mathrm{C}=\mathrm{O}) \mathrm{cm}^{-1}$; MS(FAB): $m / z 392.3\left[\mathrm{M}+\mathrm{Na}^{+}\right]$.

(R)-2-NPP amide of L-phenylalaninol $(10, \mathrm{R}=(R)$-2-NPP)

$[\alpha]_{\mathrm{D}}{ }^{25}=+752\left(c=1.0\right.$ in $\left.\mathrm{CHCl}_{3}\right) ;{ }^{1} \mathrm{H}$ NMR $\left(300 \mathrm{MHz}, \mathrm{CDCl}_{3}, 25^{\circ} \mathrm{C}, \mathrm{TMS}\right): \delta=7.76(\mathrm{dd}$, $\left.{ }^{3} J(\mathrm{H}, \mathrm{H})=8.1 \mathrm{~Hz}, \quad{ }^{4} J(\mathrm{H}, \mathrm{H})=1.7 \mathrm{~Hz}, \quad 1 \mathrm{H}\right), 7.28 \quad\left(\mathrm{ddd}, \quad{ }^{3} J(\mathrm{H}, \mathrm{H})=8.5 \mathrm{~Hz}, \quad{ }^{3} J(\mathrm{H}, \mathrm{H})=7.2 \mathrm{~Hz}\right.$, $\left.{ }^{4} J(\mathrm{H}, \mathrm{H})=1.7 \mathrm{~Hz}, 1 \mathrm{H}\right), 6.97-7.02(\mathrm{~m}, 3 \mathrm{H}), 6.85-6.93(\mathrm{~m}, 4 \mathrm{H}), 6.80\left(\mathrm{~d},{ }^{3} J(\mathrm{H}, \mathrm{H})=8.5 \mathrm{~Hz}, 1 \mathrm{H}\right)$, 4.28-4.33 (m, 1H), 4.07-4.20 (m, 1H), 3.66-3.77 (m, 1H), 3.55-3.64 (m, 2H), 2.73-2.82 (m, 2H), 2.51-2.62 (m, 3H), 1.72-2.13 ppm (m, 3H); IR $\left(\mathrm{CCl}_{4}\right): 3375(\mathrm{NH}), 1677(\mathrm{C}=\mathrm{O}) \mathrm{cm}^{-1}$; $\mathrm{MS}(\mathrm{FAB}): m / z 392.2\left[\mathrm{M}+\mathrm{Na}^{+}\right]$.

(S)-2-NPP amide of $(\boldsymbol{R})$ - $\alpha, 4$-dimethylbenzylamine $(11, \mathrm{R}=(S)$-2-NPP) $[\alpha]_{\mathrm{D}}{ }^{25}=-1170\left(c=1.0\right.$ in $\left.\mathrm{CHCl}_{3}\right) ;{ }^{1} \mathrm{H}$ NMR $\left(300 \mathrm{MHz}, \mathrm{CDCl}_{3}, 25^{\circ} \mathrm{C}, \mathrm{TMS}\right): \delta=7.79$ (dd, $\left.{ }^{3} J(\mathrm{H}, \mathrm{H})=8.1 \mathrm{~Hz}, \quad{ }^{4} J(\mathrm{H}, \mathrm{H})=1.7 \mathrm{~Hz}, \quad 1 \mathrm{H}\right), 7.44 \quad\left(\mathrm{ddd},{ }^{3} J(\mathrm{H}, \mathrm{H})=8.5 \mathrm{~Hz}, \quad{ }^{3} J(\mathrm{H}, \mathrm{H})=7.2 \mathrm{~Hz}\right.$, $\left.{ }^{4} J(\mathrm{H}, \mathrm{H})=1.7 \mathrm{~Hz}, \quad 1 \mathrm{H}\right), 7.14 \quad(\mathrm{~s}, \quad 4 \mathrm{H}), 7.06 \quad\left(\mathrm{~d},{ }^{3} J(\mathrm{H}, \mathrm{H})=8.5 \mathrm{~Hz}, 1 \mathrm{H}\right), 7.05 \quad$ (br $\mathrm{d}$, $\left.{ }^{3} J(\mathrm{H}, \mathrm{H})=7.0 \mathrm{~Hz}, 1 \mathrm{H} ; \mathrm{NH}\right), 6.95\left(\mathrm{ddd},{ }^{3} J(\mathrm{H}, \mathrm{H})=8.1 \mathrm{~Hz},{ }^{3} J(\mathrm{H}, \mathrm{H})=7.2 \mathrm{~Hz},{ }^{4} J(\mathrm{H}, \mathrm{H})=1.0 \mathrm{~Hz}\right.$, $1 \mathrm{H}), 4.92-5.01(\mathrm{~m}, 1 \mathrm{H}), 4.38-4.43(\mathrm{~m}, 1 \mathrm{H}), 3.58-3.66(\mathrm{~m}, 1 \mathrm{H}), 2.77-2.84(\mathrm{~m} ., 1 \mathrm{H}), 2.48-$ $2.56(\mathrm{~m}, 1 \mathrm{H}), 2.33(\mathrm{~s}, 3 \mathrm{H}), 1.87-2.01(\mathrm{~m}, 3 \mathrm{H}), 1.19 \mathrm{ppm}\left(\mathrm{d},{ }^{3} J(\mathrm{H}, \mathrm{H})=6.8 \mathrm{~Hz}, 3 \mathrm{H}\right)$; IR (CCl4): $3384(\mathrm{NH}), 1679(\mathrm{C}=\mathrm{O}) \mathrm{cm}^{-1}$; MS(FAB): $m / z 376.3\left[\mathrm{M}+\mathrm{Na}^{+}\right]$. 
( $R$ )-2-NPP amide of $(\boldsymbol{R})$ - $\alpha, 4$-dimethylbenzylamine $(11, \mathrm{R}=(R)$-2-NPP)

$[\alpha]_{\mathrm{D}}{ }^{25}=+1100\left(c=1.0\right.$ in $\left.\mathrm{CHCl}_{3}\right) ;{ }^{1} \mathrm{H}$ NMR $\left(300 \mathrm{MHz}, \mathrm{CDCl}_{3}, 25^{\circ} \mathrm{C}, \mathrm{TMS}\right): \delta=7.75$ (dd, $\left.{ }^{3} J(\mathrm{H}, \mathrm{H})=8.1 \mathrm{~Hz},{ }^{4} J(\mathrm{H}, \mathrm{H})=1.7 \mathrm{~Hz}, 1 \mathrm{H}\right), 7.32 \quad\left(\mathrm{ddd},{ }^{3} J(\mathrm{H}, \mathrm{H})=8.5 \mathrm{~Hz},{ }^{3} J(\mathrm{H}, \mathrm{H})=7.2 \mathrm{~Hz}\right.$, $\left.{ }^{4} J(\mathrm{H}, \mathrm{H})=1.7 \mathrm{~Hz}, 1 \mathrm{H}\right), 7.05\left(\right.$ br d, ${ }^{3} J(\mathrm{H}, \mathrm{H})=7.0 \mathrm{~Hz}, 1 \mathrm{H}$; NH), $6.94\left(\mathrm{~d},{ }^{3} J(\mathrm{H}, \mathrm{H})=8.5 \mathrm{~Hz}, 1 \mathrm{H}\right)$, $6.92\left(\mathrm{ddd},{ }^{3} J(\mathrm{H}, \mathrm{H})=8.1 \mathrm{~Hz},{ }^{3} J(\mathrm{H}, \mathrm{H})=7.2 \mathrm{~Hz},{ }^{4} J(\mathrm{H}, \mathrm{H})=1.0 \mathrm{~Hz}, 1 \mathrm{H}\right), 6.87\left(\mathrm{~d},{ }^{3} J(\mathrm{H}, \mathrm{H})=8.0 \mathrm{~Hz}\right.$, $2 \mathrm{H}), 6.74\left(\mathrm{~d},{ }^{3} J(\mathrm{H}, \mathrm{H})=8.0 \mathrm{~Hz}, 2 \mathrm{H}\right), 4.91-5.01(\mathrm{~m}, 1 \mathrm{H}), 4.36-4.42(\mathrm{~m}, 1 \mathrm{H}), 3.63-3.71(\mathrm{~m}$, $1 \mathrm{H}), 2.80-2.87(\mathrm{~m}, 1 \mathrm{H}), 2.56-2.65(\mathrm{~m}, 1 \mathrm{H}), 2.22(\mathrm{~s}, 3 \mathrm{H}), 1.85-2.11(\mathrm{~m}, 3 \mathrm{H}), 1.40 \mathrm{ppm}(\mathrm{d}$, $\left.{ }^{3} J(\mathrm{H}, \mathrm{H})=7.0 \mathrm{~Hz}, 3 \mathrm{H}\right)$; IR $\left(\mathrm{CCl}_{4}\right): 3382(\mathrm{NH}), 1679(\mathrm{C}=\mathrm{O}) \mathrm{cm}^{-1}$; MS(FAB): $m / z 376.3$ $\left[\mathrm{M}+\mathrm{Na}^{+}\right]$.

(S)-2-NPP amide of D-alanine methyl ester $(12, \mathrm{R}=(S)$-2-NPP)

$[\alpha]_{\mathrm{D}}{ }^{25}=-743(c=1.0$ in $\mathrm{MeOH}) ;{ }^{1} \mathrm{H}$ NMR $\left(300 \mathrm{MHz}, \mathrm{CDCl}_{3}, 25^{\circ} \mathrm{C}, \mathrm{TMS}\right): \delta=7.77$ (dd, $\left.{ }^{3} J(\mathrm{H}, \mathrm{H})=8.1 \mathrm{~Hz}, \quad{ }^{4} J(\mathrm{H}, \mathrm{H})=1.7 \mathrm{~Hz}, \quad 1 \mathrm{H}\right), 7.40 \quad\left(\mathrm{ddd}, \quad{ }^{3} J(\mathrm{H}, \mathrm{H})=8.5 \mathrm{~Hz}, \quad{ }^{3} J(\mathrm{H}, \mathrm{H})=7.2 \mathrm{~Hz}\right.$, $\left.{ }^{4} J(\mathrm{H}, \mathrm{H})=1.7 \mathrm{~Hz}, 1 \mathrm{H}\right), 7.13\left(\right.$ br d, $\left.{ }^{3} J(\mathrm{H}, \mathrm{H})=7.5 \mathrm{~Hz}, 1 \mathrm{H} ; \mathrm{NH}\right), 7.00\left(\mathrm{dd},{ }^{3} J(\mathrm{H}, \mathrm{H})=8.5 \mathrm{~Hz}\right.$, $\left.{ }^{3} J(\mathrm{H}, \mathrm{H})=1.0 \mathrm{~Hz}, 1 \mathrm{H}\right), 6.92\left(\mathrm{ddd},{ }^{3} J(\mathrm{H}, \mathrm{H})=8.1 \mathrm{~Hz},{ }^{3} J(\mathrm{H}, \mathrm{H})=7.2 \mathrm{~Hz},{ }^{4} J(\mathrm{H}, \mathrm{H})=1.0 \mathrm{~Hz}, 1 \mathrm{H}\right)$, 4.39-4.49 (m, 2H), $3.74(\mathrm{~s}, 3 \mathrm{H}), 3.69\left(\mathrm{dd},{ }^{2} J(\mathrm{H}, \mathrm{H})=9.7 \mathrm{~Hz},{ }^{3} J(\mathrm{H}, \mathrm{H})=6.0 \mathrm{~Hz}, 1 \mathrm{H}\right), 2.81-2.86$ $(\mathrm{m}, 1 \mathrm{H}), 2.51-2.58(\mathrm{~m}, 1 \mathrm{H}), 2.04-2.13(\mathrm{~m}, 2 \mathrm{H}), 1.84-1.95(\mathrm{~m}, 1 \mathrm{H}), 1.24 \mathrm{ppm}(\mathrm{d}$, $\left.{ }^{3} J(\mathrm{H}, \mathrm{H})=6.2 \mathrm{~Hz}, 3 \mathrm{H}\right)$; IR $\left(\mathrm{CCl}_{4}\right): 3382(\mathrm{NH}), 1751,1685(\mathrm{C}=\mathrm{O}) \mathrm{cm}^{-1}$; MS(FAB): $m / z 344.1$ $\left[\mathrm{M}+\mathrm{Na}^{+}\right]$.

(R)-2-NPP amide of D-alanine methyl ester $(12, \mathrm{R}=(R)-2-\mathrm{NPP})$ $[\alpha]_{\mathrm{D}}{ }^{25}=+922(c=1$ in $\mathrm{MeOH}) ;{ }^{1} \mathrm{H} \mathrm{NMR}\left(300 \mathrm{MHz}, \mathrm{CDCl}_{3}, 25^{\circ} \mathrm{C}, \mathrm{TMS}\right): \delta=7.79$ (dd, $\left.{ }^{3} J(\mathrm{H}, \mathrm{H})=8.1 \mathrm{~Hz},{ }^{4} J(\mathrm{H}, \mathrm{H})=1.7 \mathrm{~Hz}, 1 \mathrm{H}\right), 7.42 \quad\left(\mathrm{ddd},{ }^{3} J(\mathrm{H}, \mathrm{H})=8.5 \mathrm{~Hz},{ }^{3} J(\mathrm{H}, \mathrm{H})=7.2 \mathrm{~Hz}\right.$, $\left.{ }^{4} J(\mathrm{H}, \mathrm{H})=1.7 \mathrm{~Hz}, 1 \mathrm{H}\right), 7.18\left(\mathrm{br} \mathrm{d},{ }^{3} J(\mathrm{H}, \mathrm{H})=7.3 \mathrm{~Hz}, 1 \mathrm{H} ; \mathrm{NH}\right), 7.02\left(\mathrm{dd},{ }^{3} J(\mathrm{H}, \mathrm{H})=8.5 \mathrm{~Hz}\right.$, $\left.{ }^{3} J(\mathrm{H}, \mathrm{H})=1.0 \mathrm{~Hz}, 1 \mathrm{H}\right), 6.93\left(\mathrm{ddd},{ }^{3} J(\mathrm{H}, \mathrm{H})=8.1 \mathrm{~Hz},{ }^{3} J(\mathrm{H}, \mathrm{H})=7.2 \mathrm{~Hz},{ }^{4} J(\mathrm{H}, \mathrm{H})=1.0 \mathrm{~Hz}, 1 \mathrm{H}\right)$, 4.38-4.52 (m, 2H), 3.63-3.76 (m, 1H), $3.53(\mathrm{~s}, 3 \mathrm{H}), 2.81-2.88(\mathrm{~m} ., 1 \mathrm{H}), 2.54-2.62(\mathrm{~m}, 1 \mathrm{H})$, 1.84-2.09 (m, 3H), 1.38ppm $\left(\mathrm{d},{ }^{3} J(\mathrm{H}, \mathrm{H})=6.2 \mathrm{~Hz}, 3 \mathrm{H}\right)$; IR $\left(\mathrm{CCl}_{4}\right): 3379(\mathrm{NH}), 1751,1679$ $(\mathrm{C}=\mathrm{O}) \mathrm{cm}^{-1}$; $\mathrm{MS}(\mathrm{FAB}): \mathrm{m} / \mathrm{z} 344.2\left[\mathrm{M}+\mathrm{Na}^{+}\right]$.

(S)-2-NPP amide of (R)-1-(1-naphthyl)ethylamine (13, R = (S)-2-NPP)

$[\alpha]_{\mathrm{D}}{ }^{25}=-601\left(c=1.0\right.$ in $\left.\mathrm{CHCl}_{3}\right) ;{ }^{1} \mathrm{H}$ NMR $\left(300 \mathrm{MHz}, \mathrm{CDCl}_{3}, 25^{\circ} \mathrm{C}, \mathrm{TMS}\right): \delta=8.05-8.09(\mathrm{~m}$, 1H), 7.85-7.89 (m, 1H), 7.77-7.80 (m, 2H), 7.39-7.54 (m, 5H), 7.09-7.11 (m, 2H), 6.95 $\left(\mathrm{ddd},{ }^{3} J(\mathrm{H}, \mathrm{H})=8.1 \mathrm{~Hz},{ }^{3} J(\mathrm{H}, \mathrm{H})=7.2 \mathrm{~Hz},{ }^{4} J(\mathrm{H}, \mathrm{H})=1.0 \mathrm{~Hz}, 1 \mathrm{H}\right), 5.80-5.89(\mathrm{~m}, 1 \mathrm{H}), 4.41-4.46$ 
(m, 1H), 3.49-3.57 (m, 1H), 2.74-2.80 (m, 1H), 2.48-2.56 (m, 1H), 1.76-1.96 (m, 3H), 1.37 ppm $\left(\mathrm{d},{ }^{3} J(\mathrm{H}, \mathrm{H})=7.0 \mathrm{~Hz}, 3 \mathrm{H}\right)$; IR $\left(\mathrm{CCl}_{4}\right): 3378(\mathrm{NH}), 1677(\mathrm{C}=\mathrm{O}) \mathrm{cm}^{-1}$; MS(FAB): $\mathrm{m} / \mathrm{z}$ $412.2\left[\mathrm{M}+\mathrm{Na}^{+}\right]$.

( $R$ )-2-NPP amide of $(R)$-1-(1-naphthyl)ethylamine $(13, \mathrm{R}=(R)-2-\mathrm{NPP})$

$[\alpha]_{\mathrm{D}}{ }^{25}=+732(c=1.0$ in $\mathrm{MeOH}) ;{ }^{1} \mathrm{H}$ NMR $\left(300 \mathrm{MHz}, \mathrm{CDCl}_{3}, 25^{\circ} \mathrm{C}, \mathrm{TMS}\right): \delta=7.88(\mathrm{~d}$, $\left.{ }^{3} J(\mathrm{H}, \mathrm{H})=8.3 \mathrm{~Hz}, 1 \mathrm{H}\right), 7.73\left(\mathrm{~d},{ }^{3} J(\mathrm{H}, \mathrm{H})=7.8 \mathrm{~Hz}, 1 \mathrm{H}\right), 7.61\left(\mathrm{~d},{ }^{3} J(\mathrm{H}, \mathrm{H})=7.5 \mathrm{~Hz}, 1 \mathrm{H}\right), 7.59(\mathrm{dd}$, $\left.{ }^{3} J(\mathrm{H}, \mathrm{H})=8.1 \mathrm{~Hz},{ }^{4} J(\mathrm{H}, \mathrm{H})=1.5 \mathrm{~Hz}, 1 \mathrm{H}\right), 7.35-7.40(\mathrm{~m}, 1 \mathrm{H}), 7.33\left(\mathrm{ddd},{ }^{3} J(\mathrm{H}, \mathrm{H})=8.3 \mathrm{~Hz}\right.$, $\left.{ }^{3} J(\mathrm{H}, \mathrm{H})=6.8 \mathrm{~Hz},{ }^{4} J(\mathrm{H}, \mathrm{H})=1.6 \mathrm{~Hz}, 1 \mathrm{H}\right), 7.10-7.20(\mathrm{~m}, 3 \mathrm{H}), 6.96\left(\mathrm{~d},{ }^{3} J(\mathrm{H}, \mathrm{H})=8.5 \mathrm{~Hz}, 1 \mathrm{H}\right)$, $6.89\left(\mathrm{~d},{ }^{3} J(\mathrm{H}, \mathrm{H})=8.6 \mathrm{~Hz}, 1 \mathrm{H}\right), 6.78\left(\mathrm{ddd},{ }^{3} J(\mathrm{H}, \mathrm{H})=8.1 \mathrm{~Hz},{ }^{3} J(\mathrm{H}, \mathrm{H})=7.2 \mathrm{~Hz},{ }^{4} J(\mathrm{H}, \mathrm{H})=1.0 \mathrm{~Hz}\right.$, $1 \mathrm{H}), 5.74-5.84(\mathrm{~m}, 1 \mathrm{H}), 4.38-4.44(\mathrm{~m}, 1 \mathrm{H}), 3.59-3.68(\mathrm{~m}, 1 \mathrm{H}), 2.76-2.84(\mathrm{~m}, 1 \mathrm{H}), 2.56-$ $2.66(\mathrm{~m}, 1 \mathrm{H}), 1.84-2.12(\mathrm{~m}, 3 \mathrm{H}), 1.60 \mathrm{ppm}\left(\mathrm{d},{ }^{3} J(\mathrm{H}, \mathrm{H})=7.5 \mathrm{~Hz}, 3 \mathrm{H}\right)$; IR $\left(\mathrm{CCl}_{4}\right): 3382(\mathrm{NH})$, $1679(\mathrm{C}=\mathrm{O}) \mathrm{cm}^{-1}$; $\mathrm{MS}(\mathrm{FAB}): \mathrm{m} / z 412.3\left[\mathrm{M}+\mathrm{Na}^{+}\right]$.

(S)-2-NPP amide of ( $S)$-sec-butylamine $(14, \mathrm{R}=(S)$-2-NPP)

$[\alpha]_{\mathrm{D}}{ }^{25}=-1290\left(c=1.0\right.$ in $\left.\mathrm{CHCl}_{3}\right) ;{ }^{1} \mathrm{H}$ NMR $\left(400 \mathrm{MHz}, \mathrm{CDCl}_{3}, 25^{\circ} \mathrm{C}, \mathrm{TMS}\right): \delta=7.76(\mathrm{dd}$, $\left.{ }^{3} J(\mathrm{H}, \mathrm{H})=8.1 \mathrm{~Hz}, \quad{ }^{4} J(\mathrm{H}, \mathrm{H})=1.7 \mathrm{~Hz}, \quad 1 \mathrm{H}\right), 7.40 \quad\left(\mathrm{ddd},{ }^{3} J(\mathrm{H}, \mathrm{H})=8.5 \mathrm{~Hz}, \quad{ }^{3} J(\mathrm{H}, \mathrm{H})=7.2 \mathrm{~Hz}\right.$, $\left.{ }^{4} J(\mathrm{H}, \mathrm{H})=1.7 \mathrm{~Hz}, \quad 1 \mathrm{H}\right), \quad 7.04 \quad\left(\mathrm{~d},{ }^{3} J(\mathrm{H}, \mathrm{H})=8.5 \mathrm{~Hz}, \quad 1 \mathrm{H}\right), \quad 6.91 \quad\left(\mathrm{ddd}, \quad{ }^{3} J(\mathrm{H}, \mathrm{H})=8.1 \mathrm{~Hz}\right.$, $\left.{ }^{3} J(\mathrm{H}, \mathrm{H})=7.2 \mathrm{~Hz},{ }^{4} J(\mathrm{H}, \mathrm{H})=1.0 \mathrm{~Hz}, 1 \mathrm{H}\right), 6.54\left(\mathrm{br} \mathrm{d},{ }^{3} J(\mathrm{H}, \mathrm{H})=8.0 \mathrm{~Hz}, 1 \mathrm{H} ; \mathrm{NH}\right), 4.37-4.41(\mathrm{~m}$, $1 \mathrm{H}), 3.73-3.84(\mathrm{~m}, 1 \mathrm{H}), 3.61-3.68(\mathrm{~m}, 1 \mathrm{H}), 2.80-2.84(1 \mathrm{H}), 2.57-2.63(\mathrm{~m}, 1 \mathrm{H}), 2.01-2.07$ $(\mathrm{m}, 1 \mathrm{H}), 1.82-1.99(\mathrm{~m}, 2 \mathrm{H}), 1.25-1.36(\mathrm{~m}, 1 \mathrm{H}), 1.12-1.22(\mathrm{~m}, 1 \mathrm{H}), 1.08\left(\mathrm{~d},{ }^{3} J(\mathrm{H}, \mathrm{H})=6.6 \mathrm{~Hz}\right.$, $3 \mathrm{H}), 0.48 \mathrm{ppm}\left(\mathrm{t},{ }^{3} J(\mathrm{H}, \mathrm{H})=7.5 \mathrm{~Hz}, 3 \mathrm{H}\right)$; IR $\left(\mathrm{CCl}_{4}\right): 3381(\mathrm{NH}), 1678(\mathrm{C}=\mathrm{O}) \mathrm{cm}^{-1}$; $\mathrm{MS}(\mathrm{FAB}): m / z 314.3\left[\mathrm{M}+\mathrm{Na}^{+}\right]$.

(R)-2-NPP amide of (S)-sec-butylamine $(14, \mathrm{R}=(R)$-2-NPP)

$[\alpha]_{\mathrm{D}}{ }^{25}=+1460\left(c=1.0\right.$ in $\left.\mathrm{CHCl}_{3}\right) ;{ }^{1} \mathrm{H}$ NMR $\left(400 \mathrm{MHz}, \mathrm{CDCl}_{3}, 25^{\circ} \mathrm{C}, \mathrm{TMS}\right): \delta=7.77(\mathrm{dd}$, $\left.{ }^{3} J(\mathrm{H}, \mathrm{H})=8.1 \mathrm{~Hz}, \quad{ }^{4} J(\mathrm{H}, \mathrm{H})=1.7 \mathrm{~Hz}, \quad 1 \mathrm{H}\right), 7.40 \quad\left(\mathrm{ddd}, \quad{ }^{3} J(\mathrm{H}, \mathrm{H})=8.5 \mathrm{~Hz}, \quad{ }^{3} J(\mathrm{H}, \mathrm{H})=7.2 \mathrm{~Hz}\right.$, $\left.{ }^{4} J(\mathrm{H}, \mathrm{H})=1.7 \mathrm{~Hz}, \quad 1 \mathrm{H}\right), \quad 7.02 \quad\left(\mathrm{~d},{ }^{3} J(\mathrm{H}, \mathrm{H})=8.5 \mathrm{~Hz}, \quad 1 \mathrm{H}\right), 6.92 \quad\left(\mathrm{ddd}, \quad{ }^{3} J(\mathrm{H}, \mathrm{H})=8.1 \mathrm{~Hz}\right.$, $\left.{ }^{3} J(\mathrm{H}, \mathrm{H})=7.2 \mathrm{~Hz},{ }^{4} J(\mathrm{H}, \mathrm{H})=1.0 \mathrm{~Hz}, 1 \mathrm{H}\right), 6.56\left(\mathrm{br} \mathrm{d},{ }^{3} J(\mathrm{H}, \mathrm{H})=7.6 \mathrm{~Hz}, 1 \mathrm{H} ; \mathrm{NH}\right), 4.36-4.40(\mathrm{~m}$, $1 \mathrm{H}), 3.77-3.84(\mathrm{~m}, 1 \mathrm{H}), 3.61-3.67(\mathrm{~m}, 1 \mathrm{H}), 2.79-2.84(\mathrm{~m}, 1 \mathrm{H}), 2.55-2.62(\mathrm{~m}, 1 \mathrm{H}), 1.85-$ $2.07(\mathrm{~m}, 3 \mathrm{H}), \quad 1.39-1.46(\mathrm{~m}, 2 \mathrm{H}), 0.88 \quad\left(\mathrm{t},{ }^{3} J(\mathrm{H}, \mathrm{H})=7.5 \mathrm{~Hz}, 3 \mathrm{H}\right), 0.85 \mathrm{ppm} \quad(\mathrm{d}$, $\left.{ }^{3} J(\mathrm{H}, \mathrm{H})=6.6 \mathrm{~Hz}, 3 \mathrm{H}\right)$; IR $\left(\mathrm{CCl}_{4}\right): 3381(\mathrm{NH}), 1679(\mathrm{C}=\mathrm{O}) \mathrm{cm}^{-1}$; $\mathrm{MS}(\mathrm{FAB}): \mathrm{m} / z 314.4$ $\left[\mathrm{M}+\mathrm{Na}^{+}\right]$. 
(S)-3
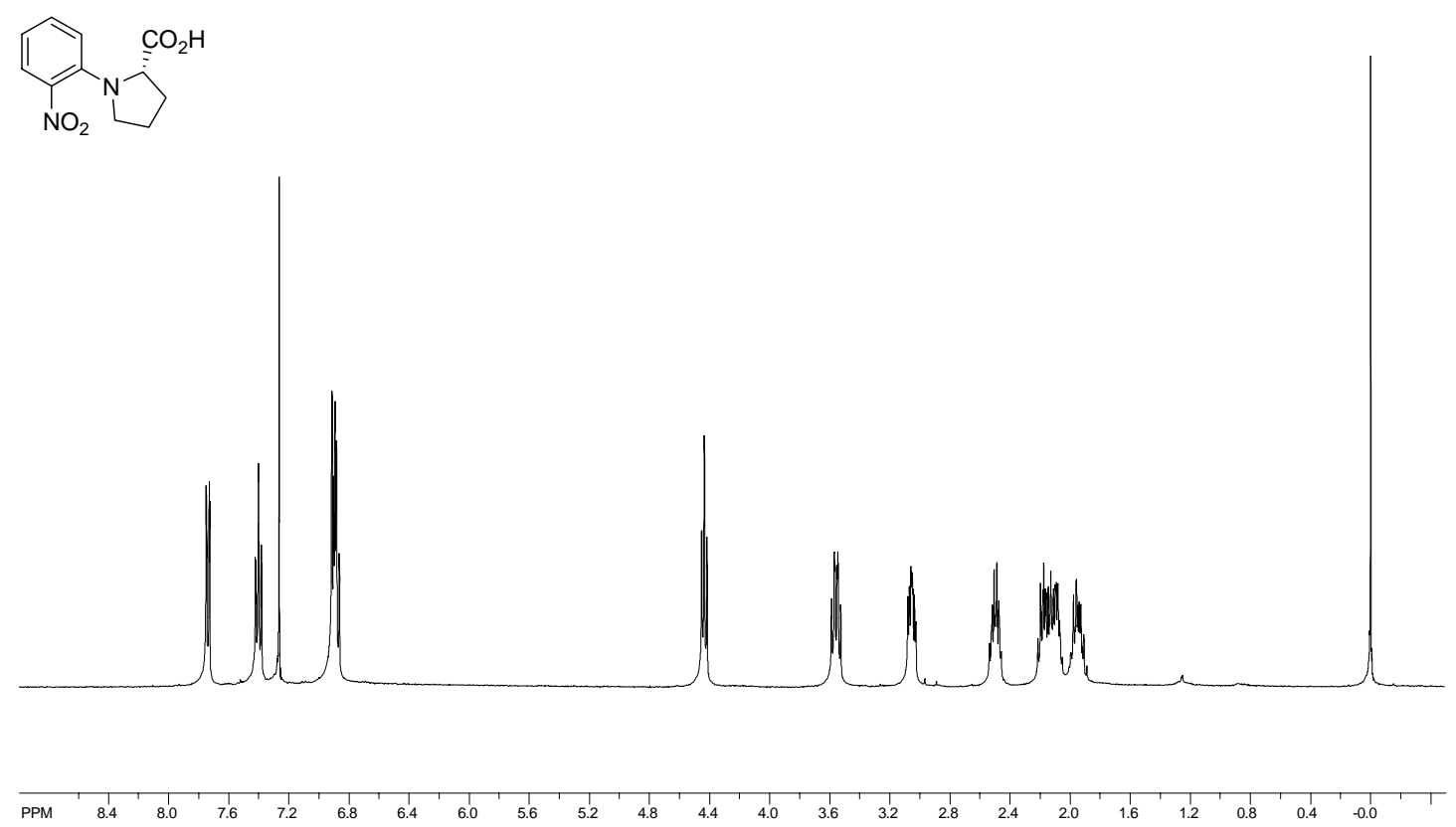

(R)-3
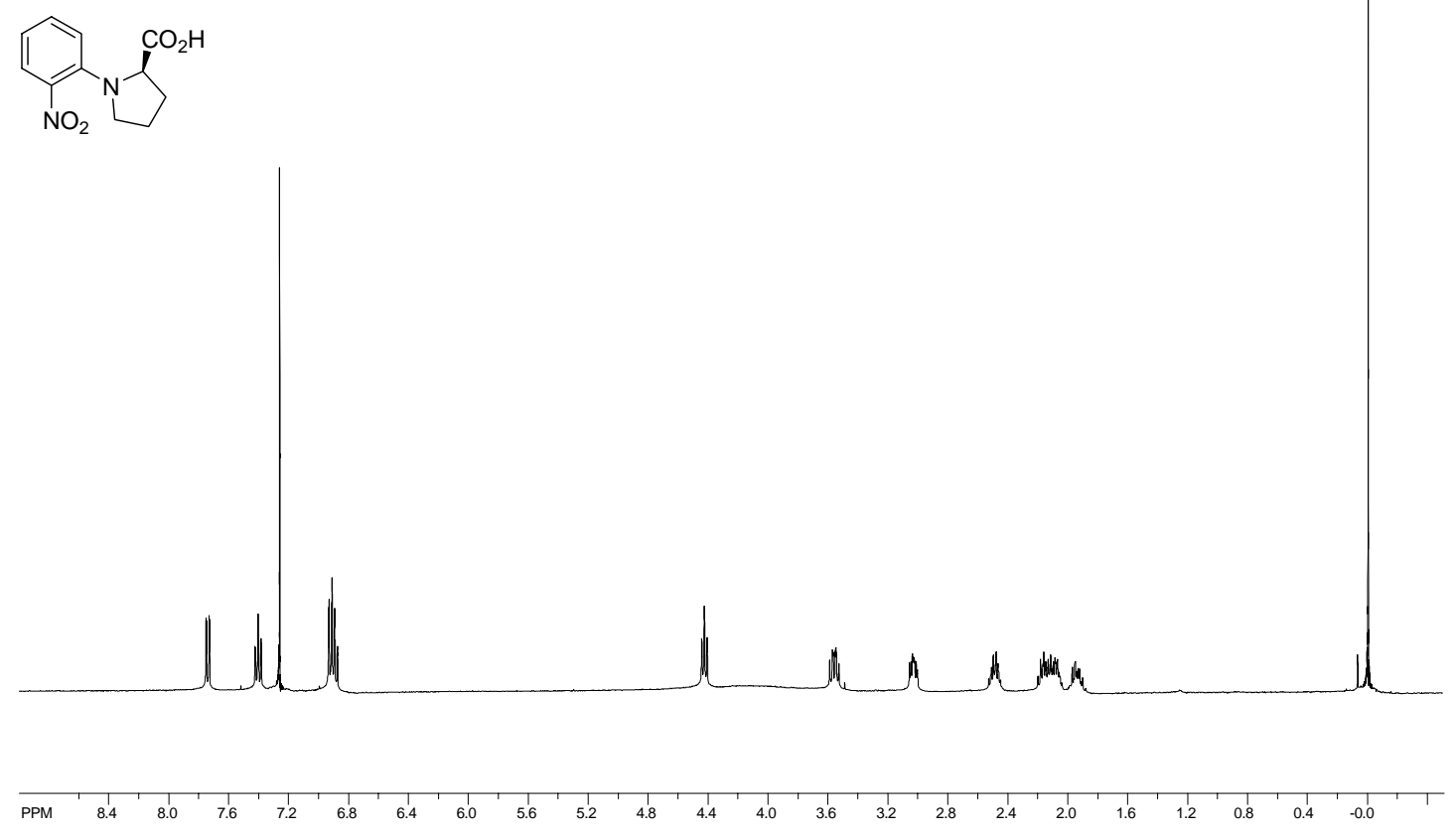

S11 
(S)-4

管
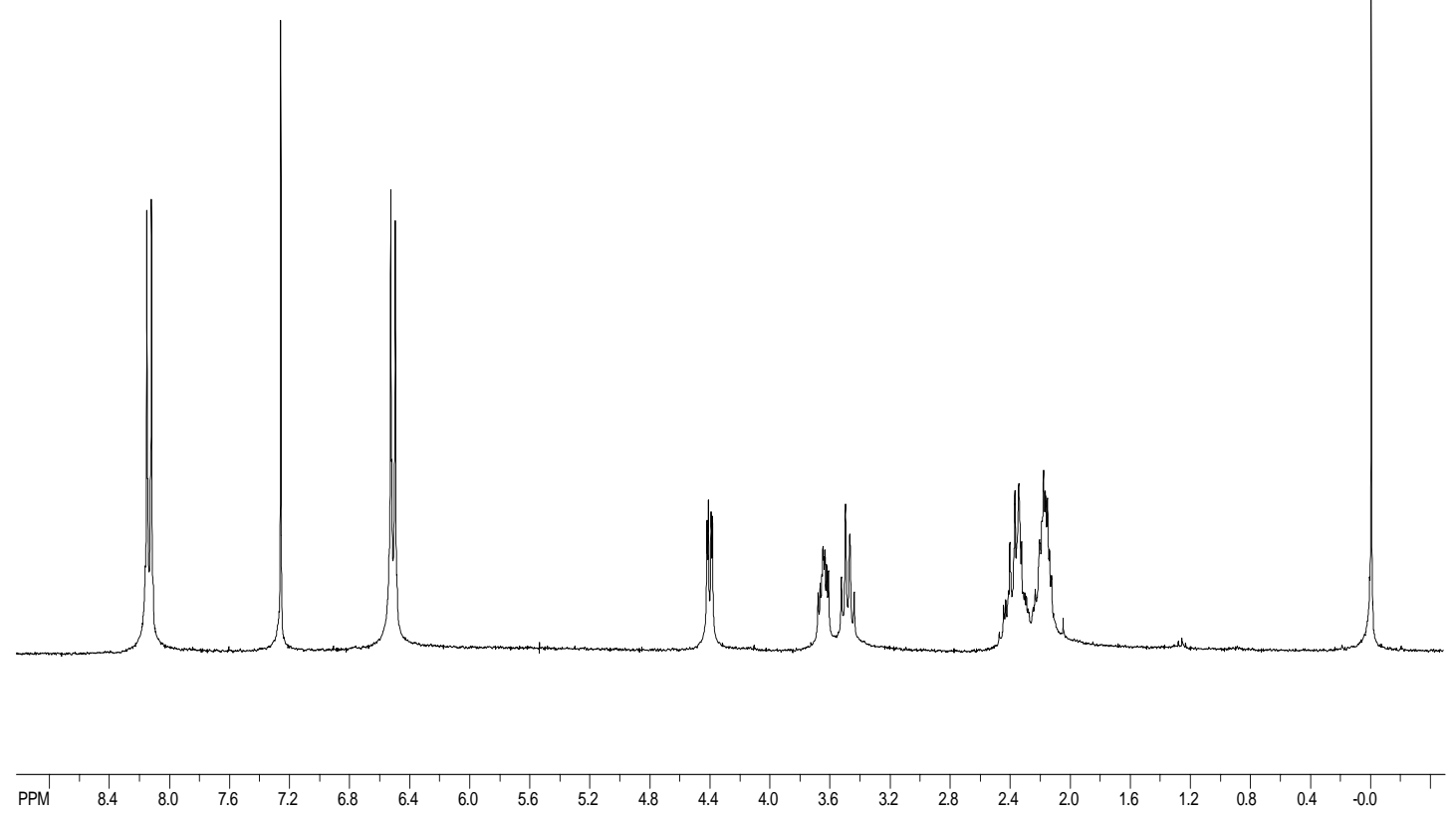

(R)-4

等

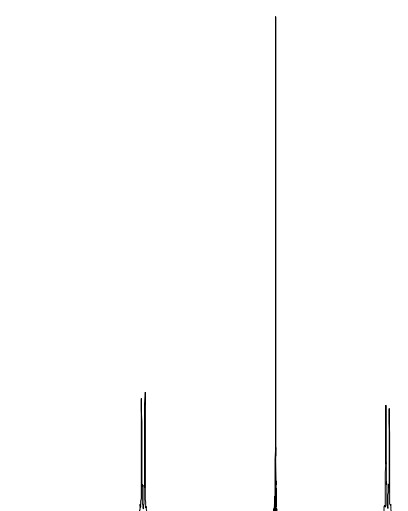

the

\begin{tabular}{llllllllllllllllllllllllllllllllllllllllll}
\hline PPM & 8.4 & 8.0 & 7.6 & 7.2 & 6.8 & 6.4 & 6.0 & 5.6 & 5.2 & 4.8 & 4.4 & 4.0 & 3.6 & 3.2 & 2.8 & 2.4 & 2.0 & 1.6 & 1.2 & 0.8 & 0.4 & 0.0 & 1 \\
\hline
\end{tabular} 
$5, \mathrm{R}=(S)-2-\mathrm{NPP}$

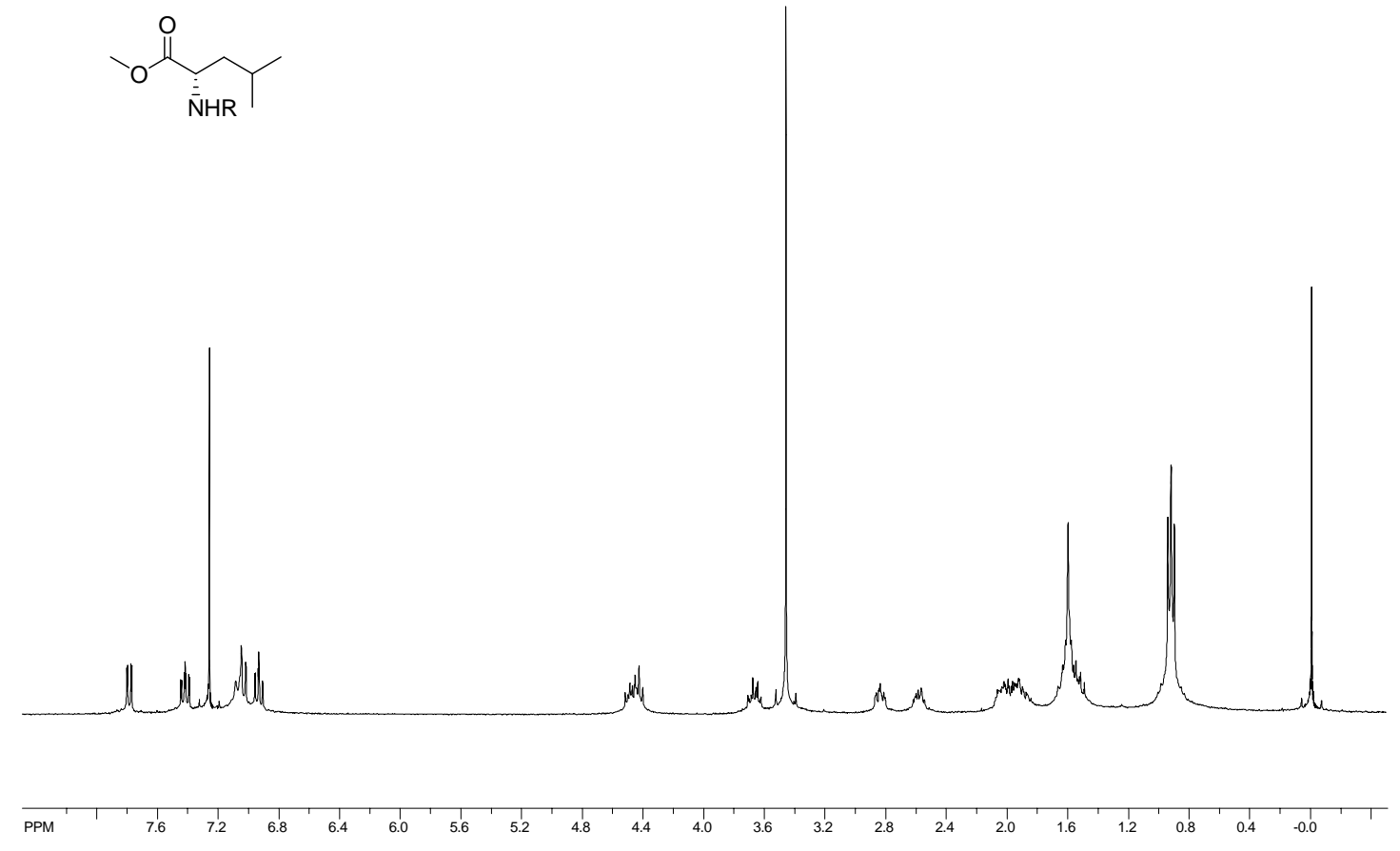

$5, \mathrm{R}=(R)-2-\mathrm{NPP}$

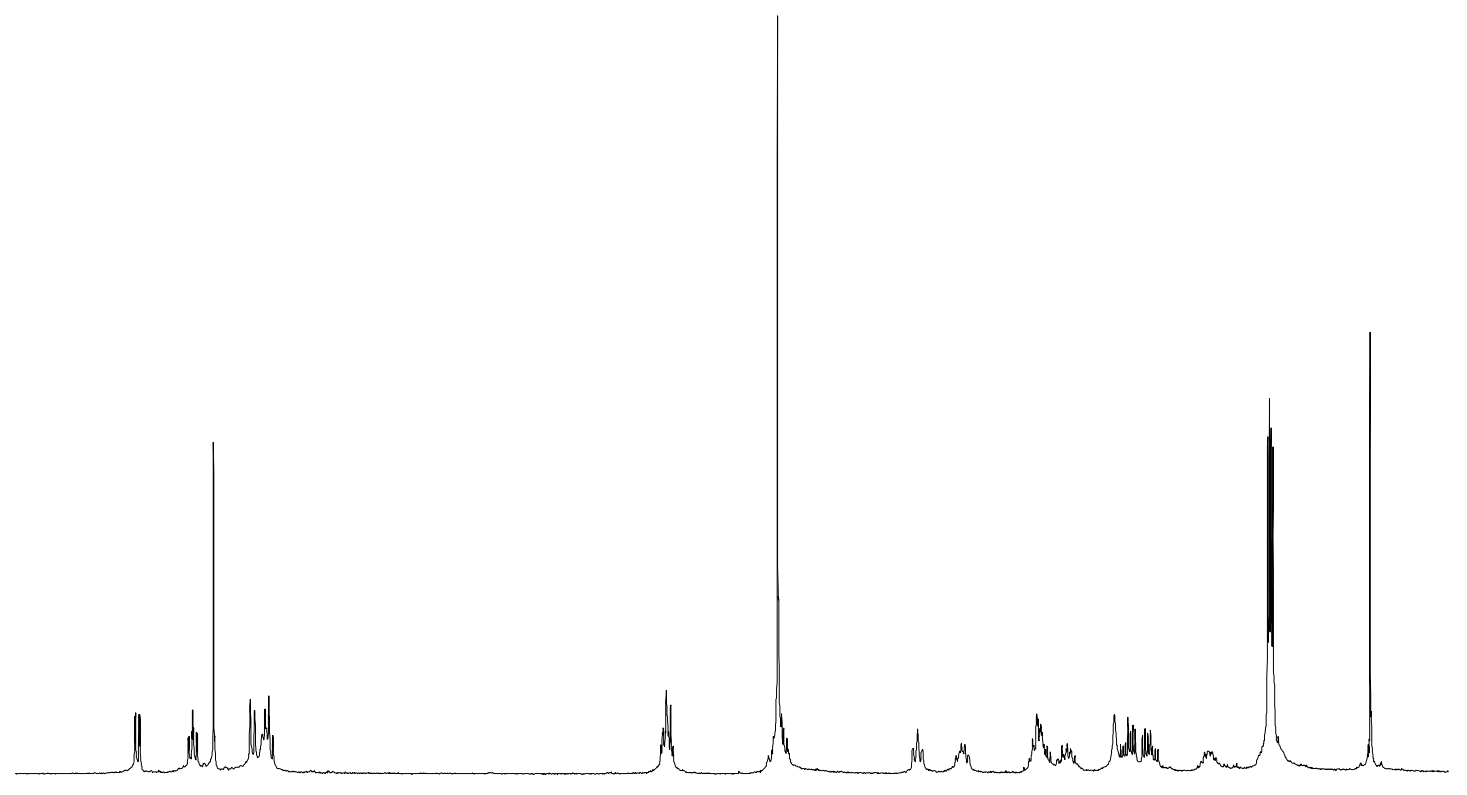

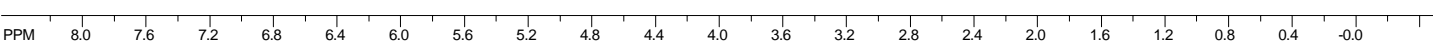


5, $\mathrm{R}=(S)-4-\mathrm{NPP}$
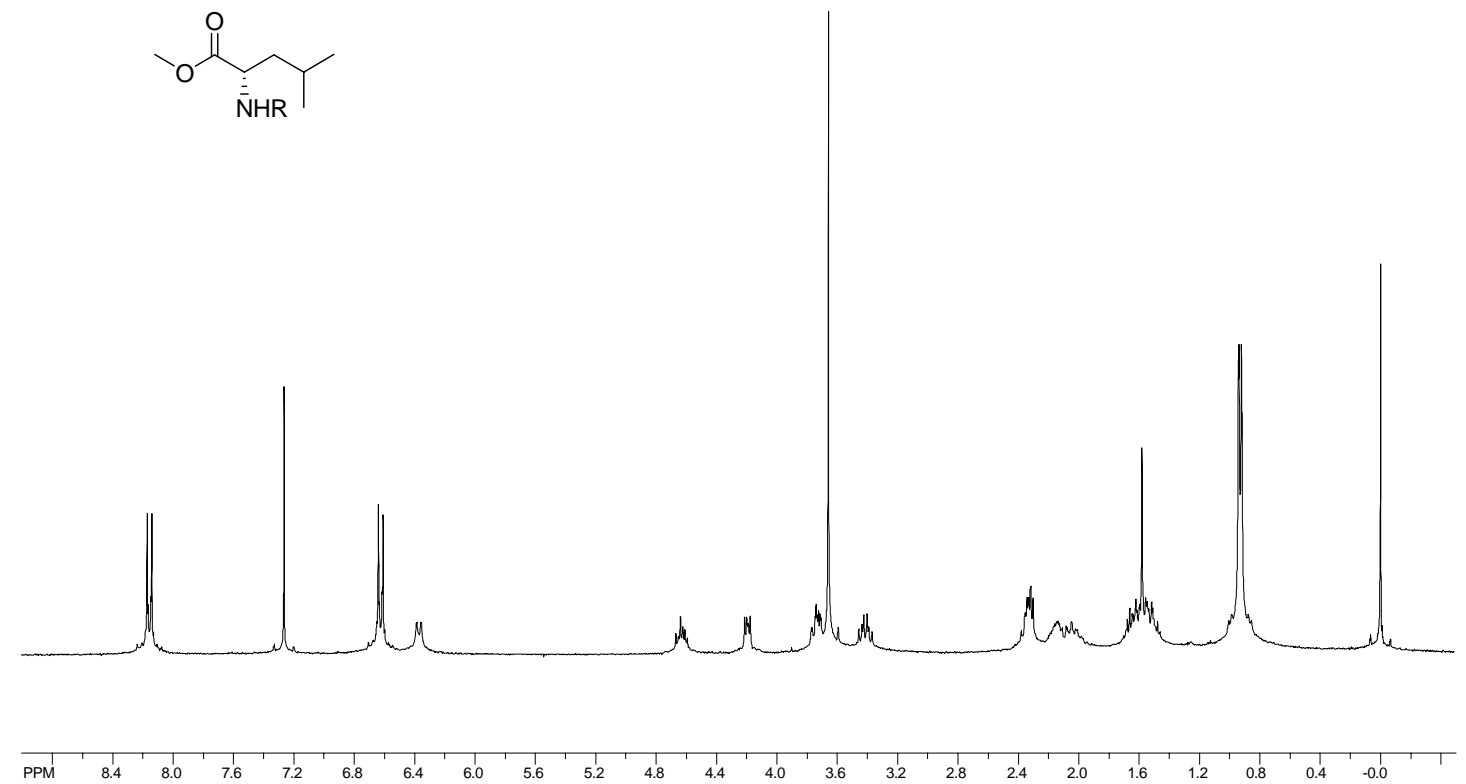

5, $\mathrm{R}=(R)-4-\mathrm{NPP}$

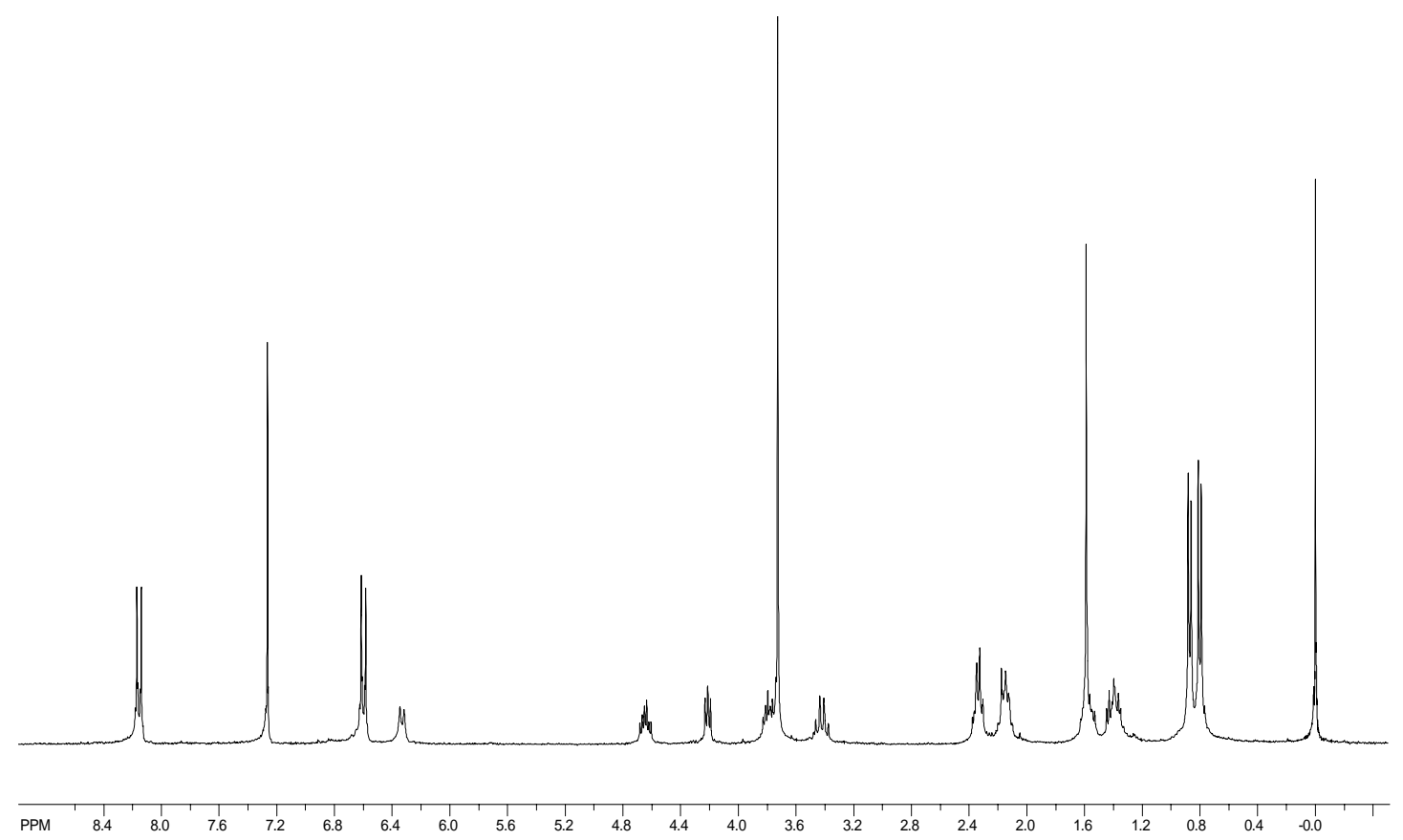


6, $\mathrm{R}=(S)-2-\mathrm{NPP}$

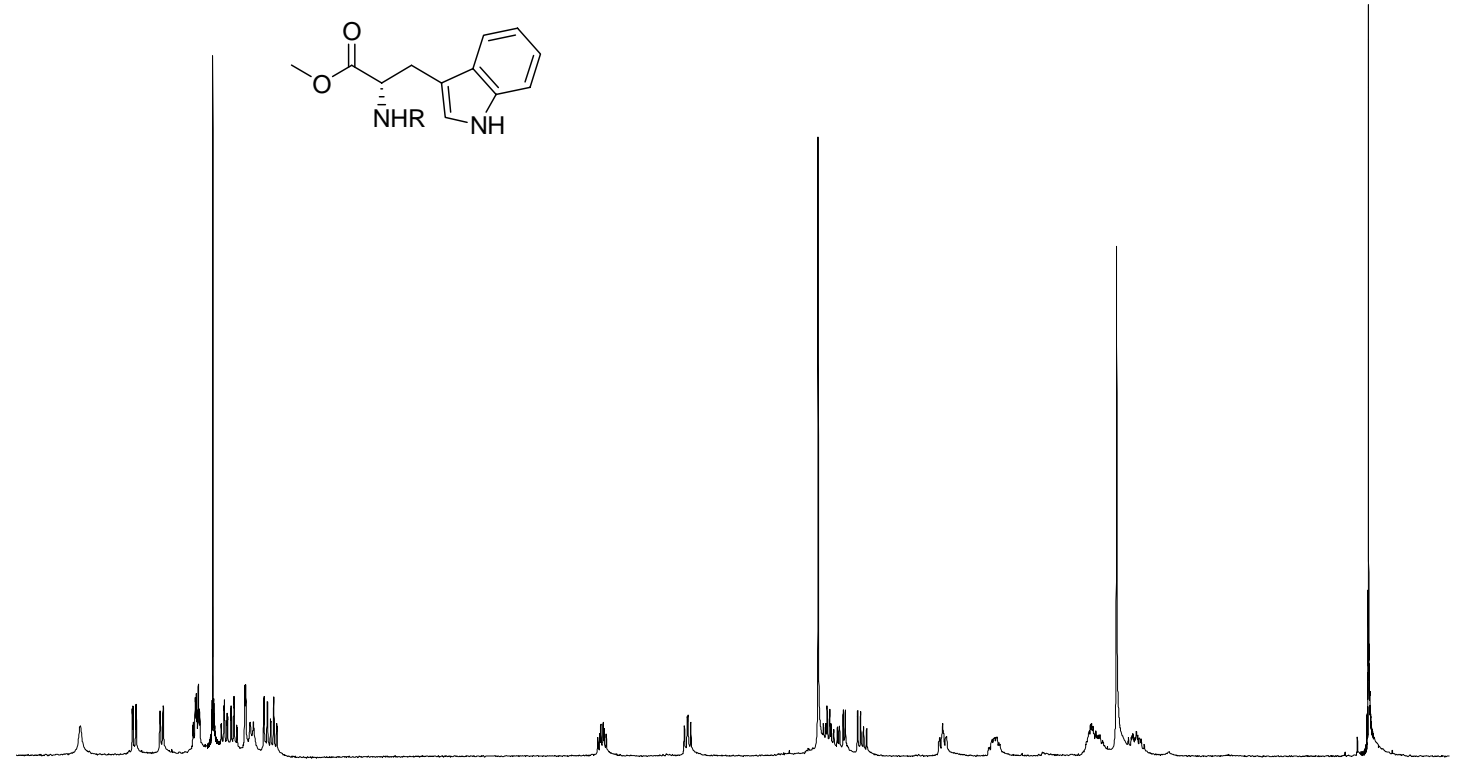

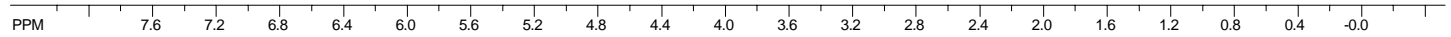

6, $\mathrm{R}=(R)-2-\mathrm{NPP}$

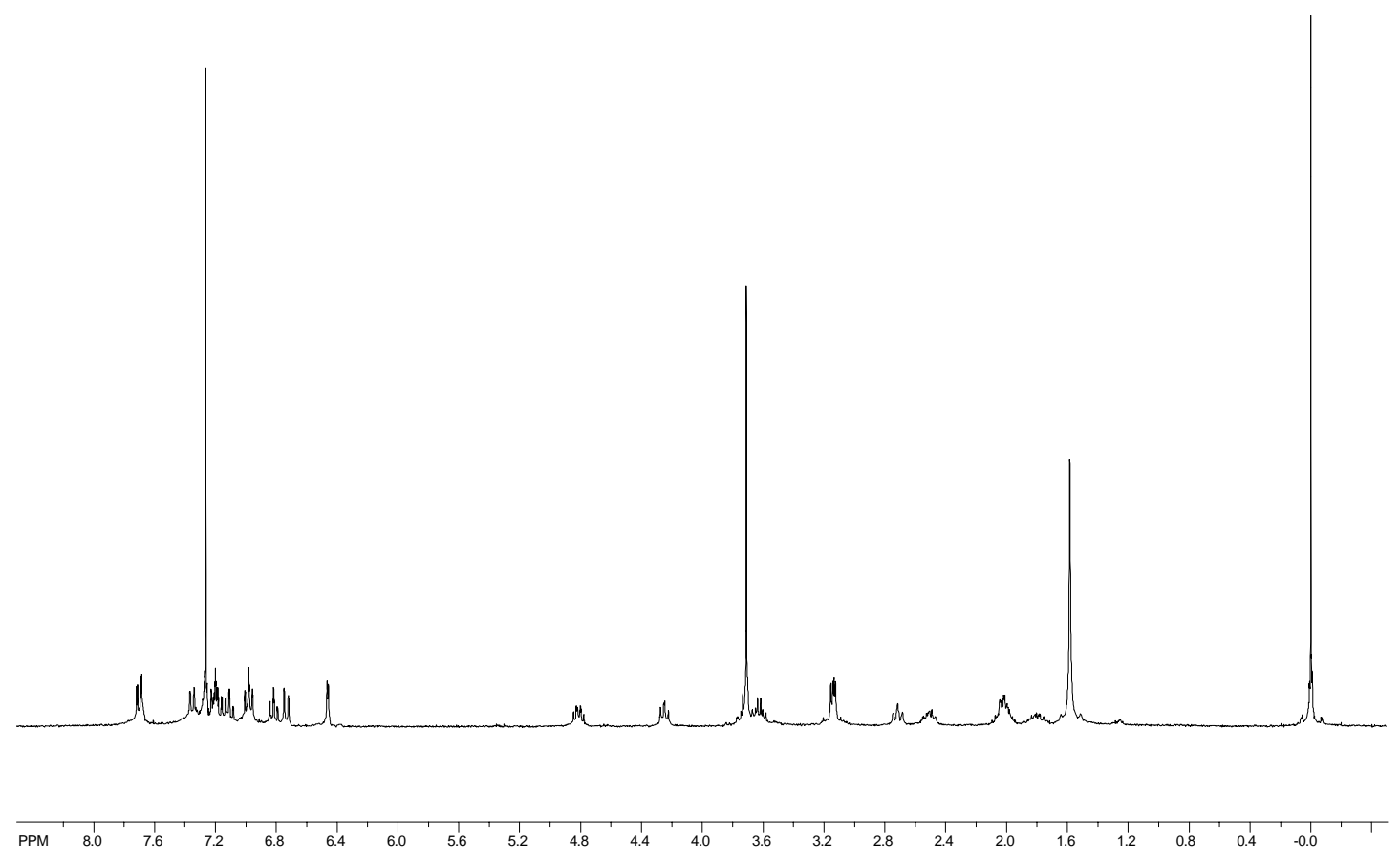


6, $\mathrm{R}=(S)-4-\mathrm{NPP}$
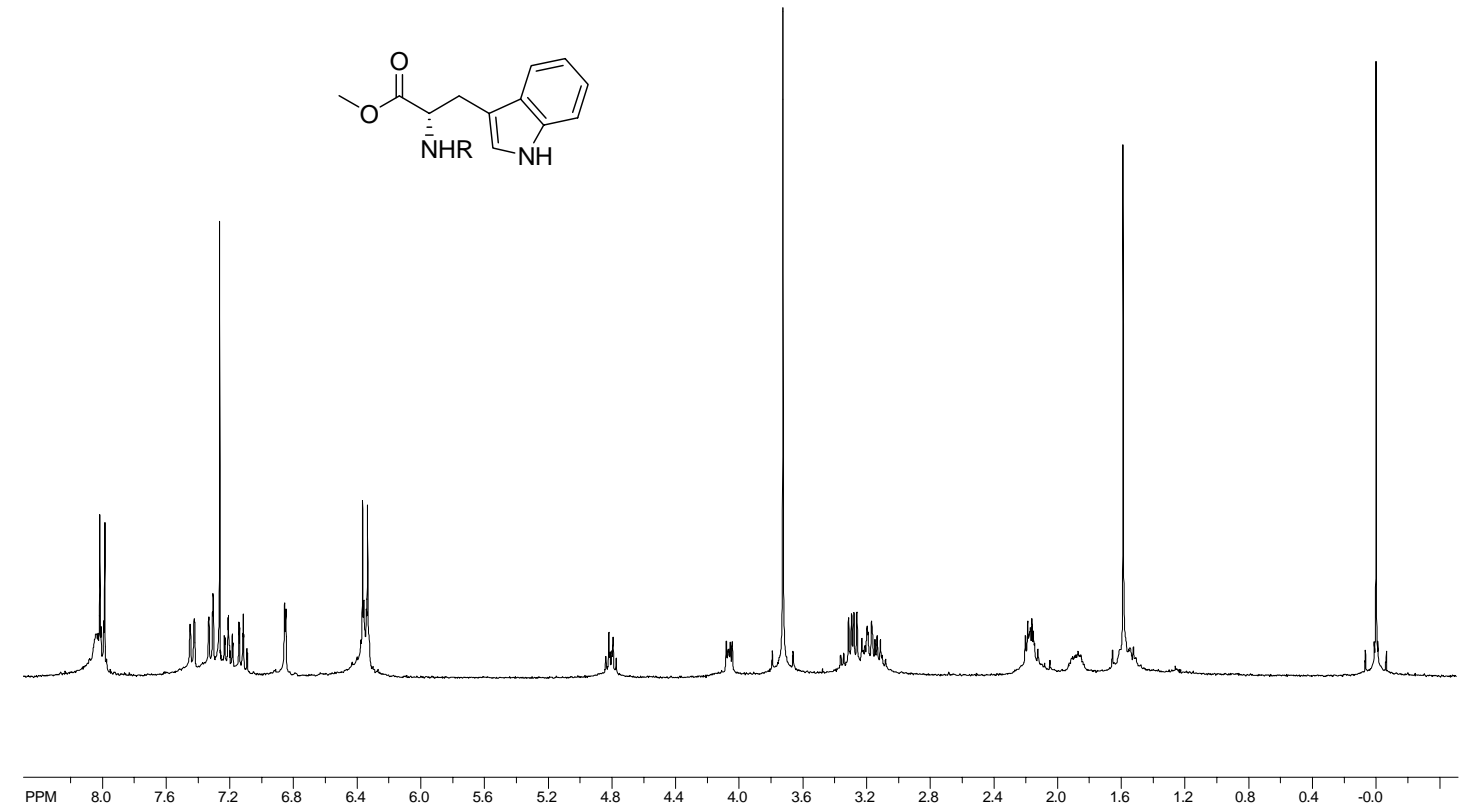

6, $\mathrm{R}=(R)-4-\mathrm{NPP}$

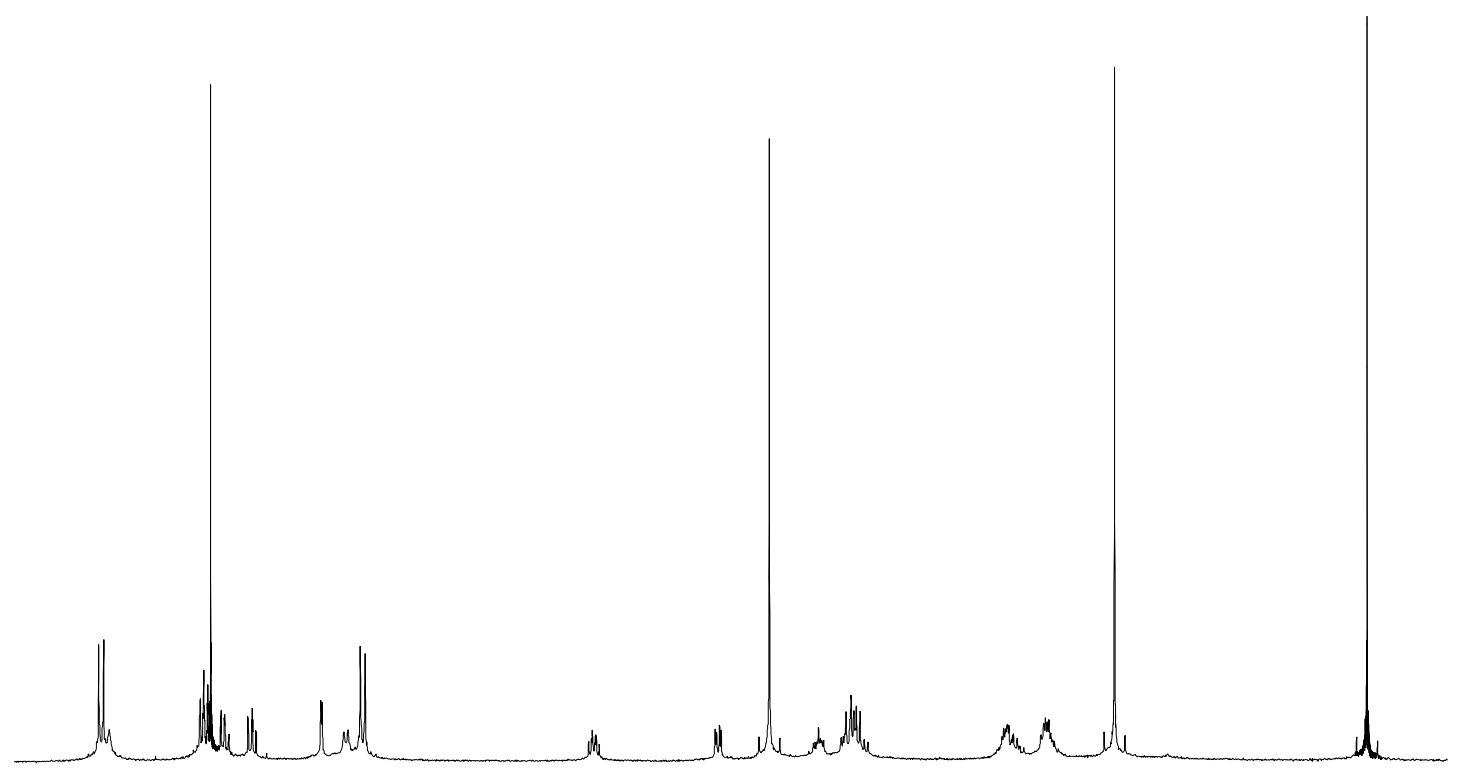


7, $\mathrm{R}=(S)-2-\mathrm{NPP}$
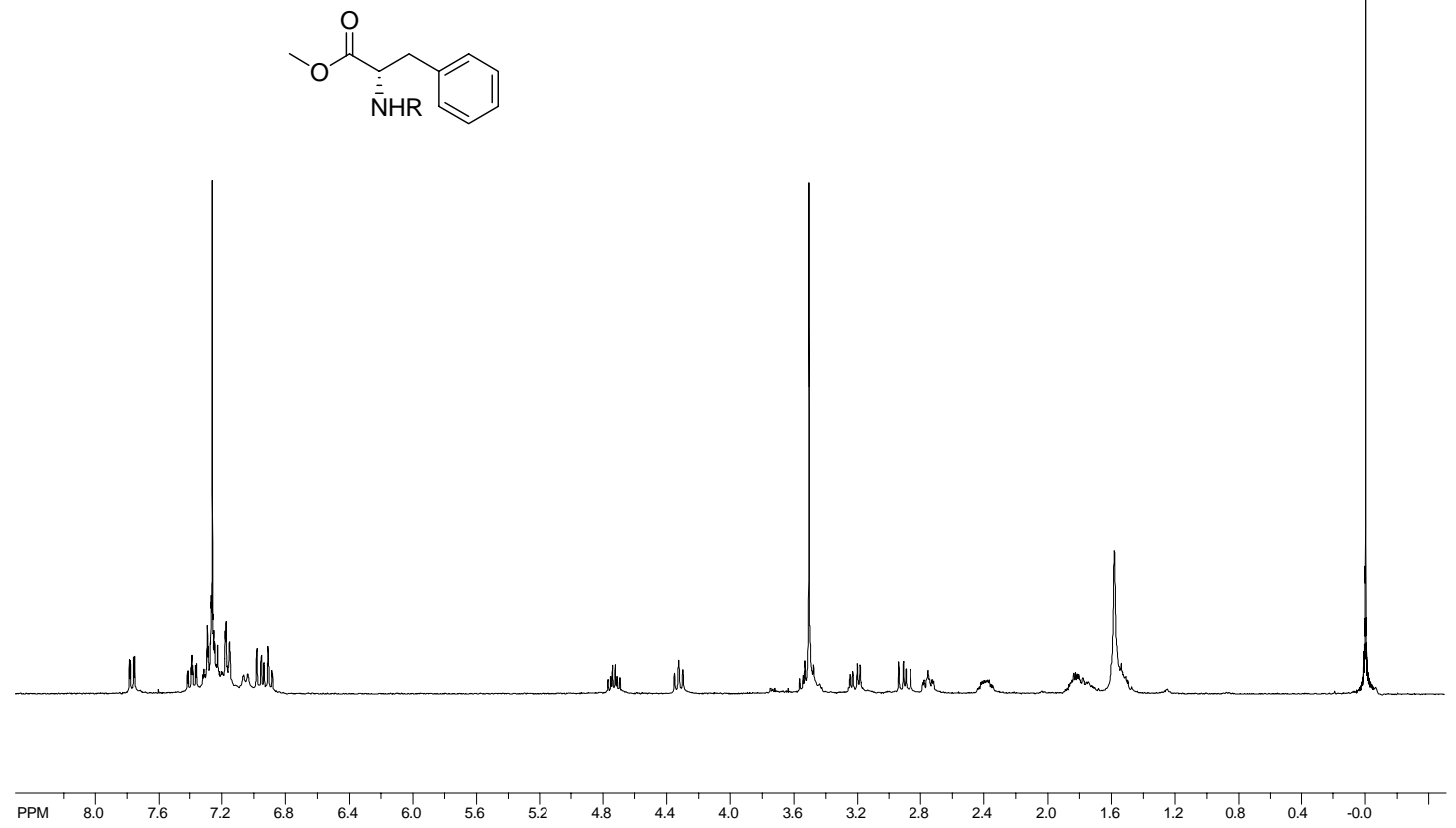

7, $\mathrm{R}=(R)-2-\mathrm{NPP}$

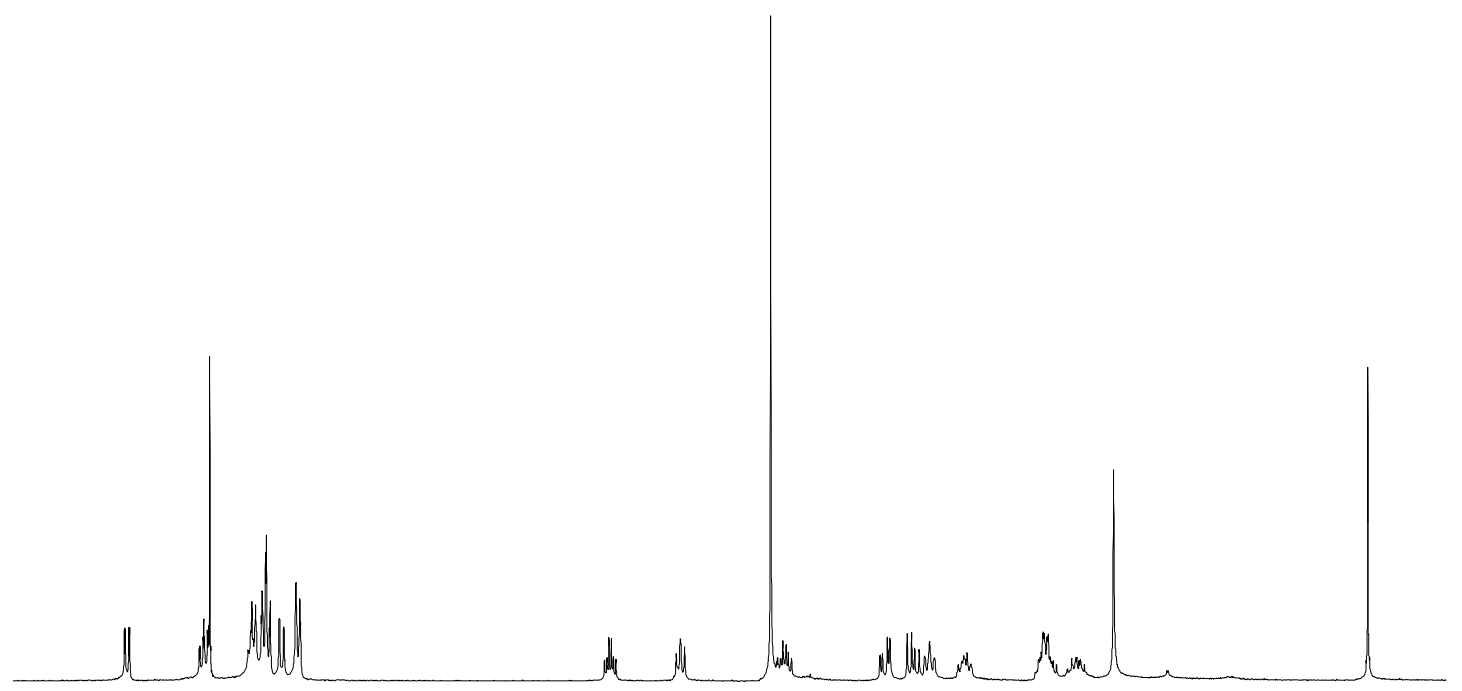


7, $\mathrm{R}=(S)-4-\mathrm{NPP}$
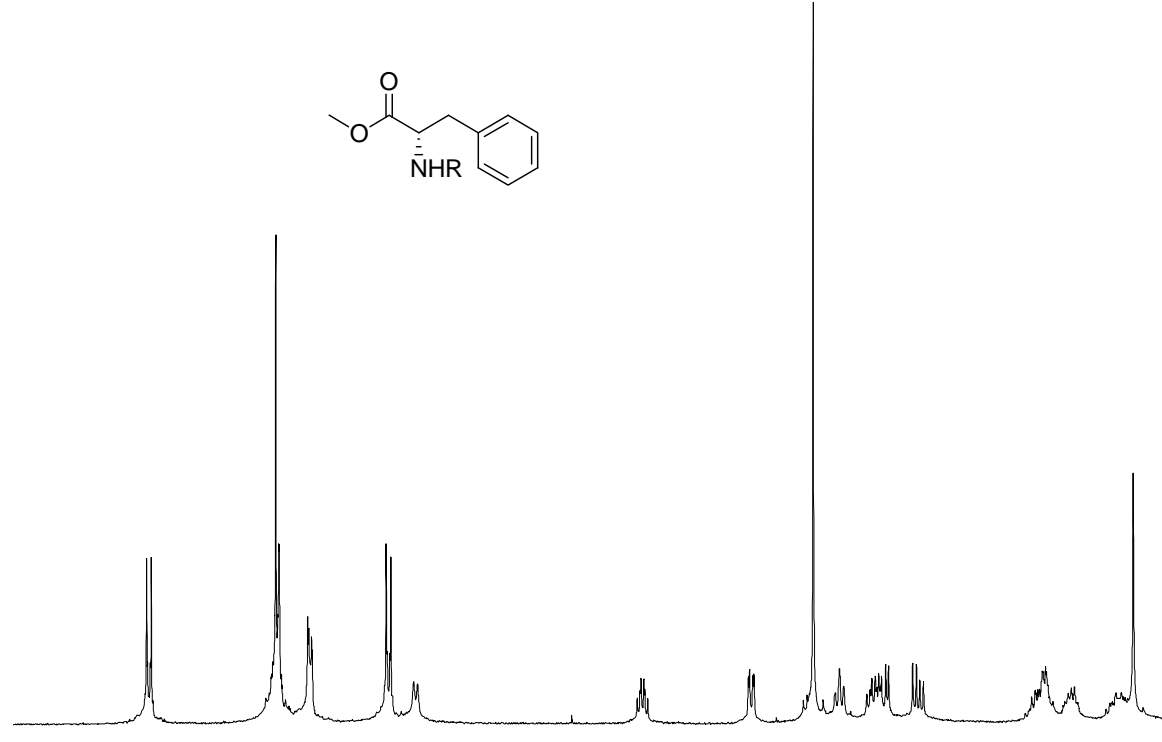

PPM
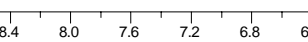

$5.2 \quad 48$

7, $\mathrm{R}=(R)-4-\mathrm{NPP}$

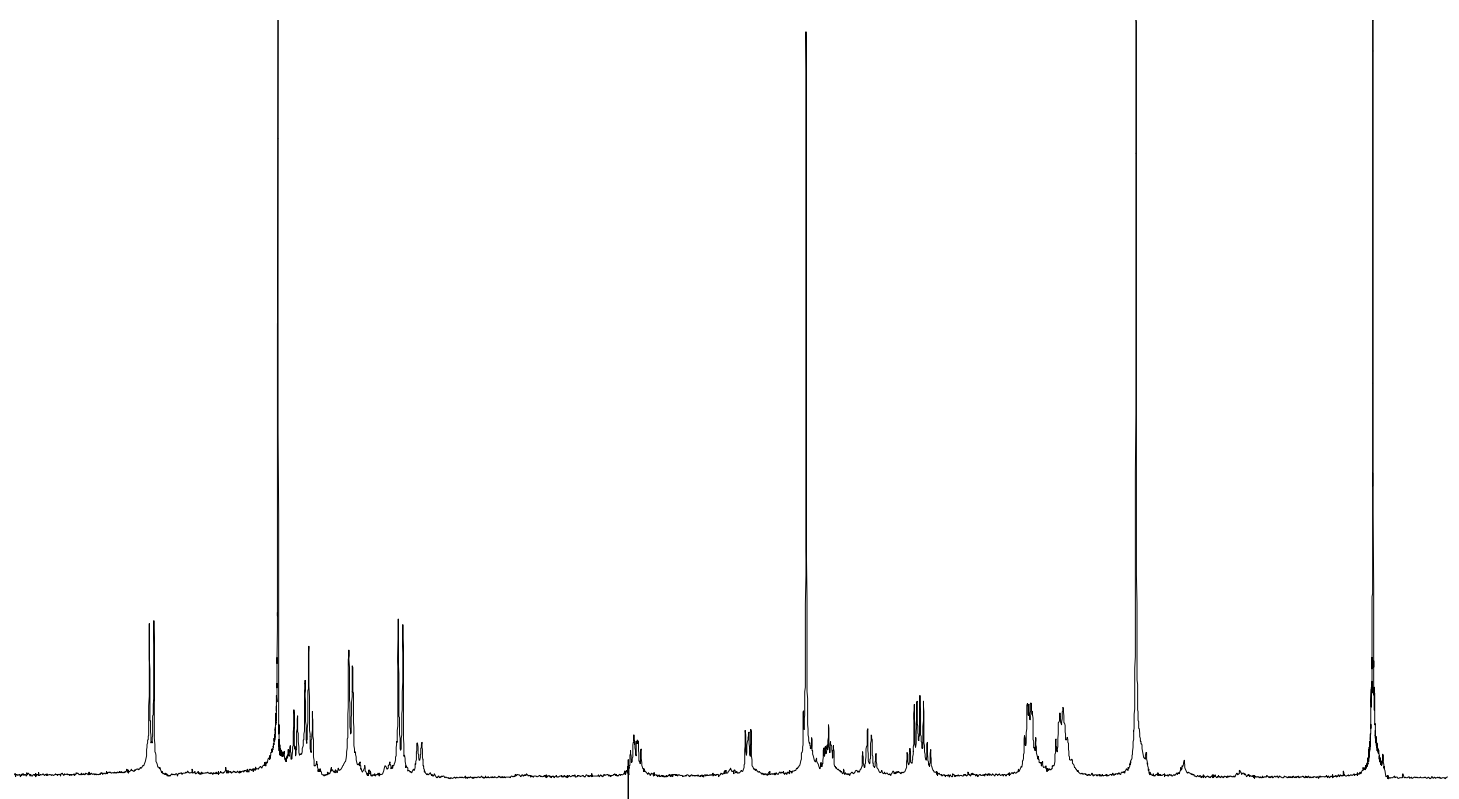

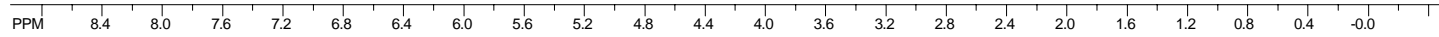


8, $\mathrm{R}=(S)-2-\mathrm{NPP}$
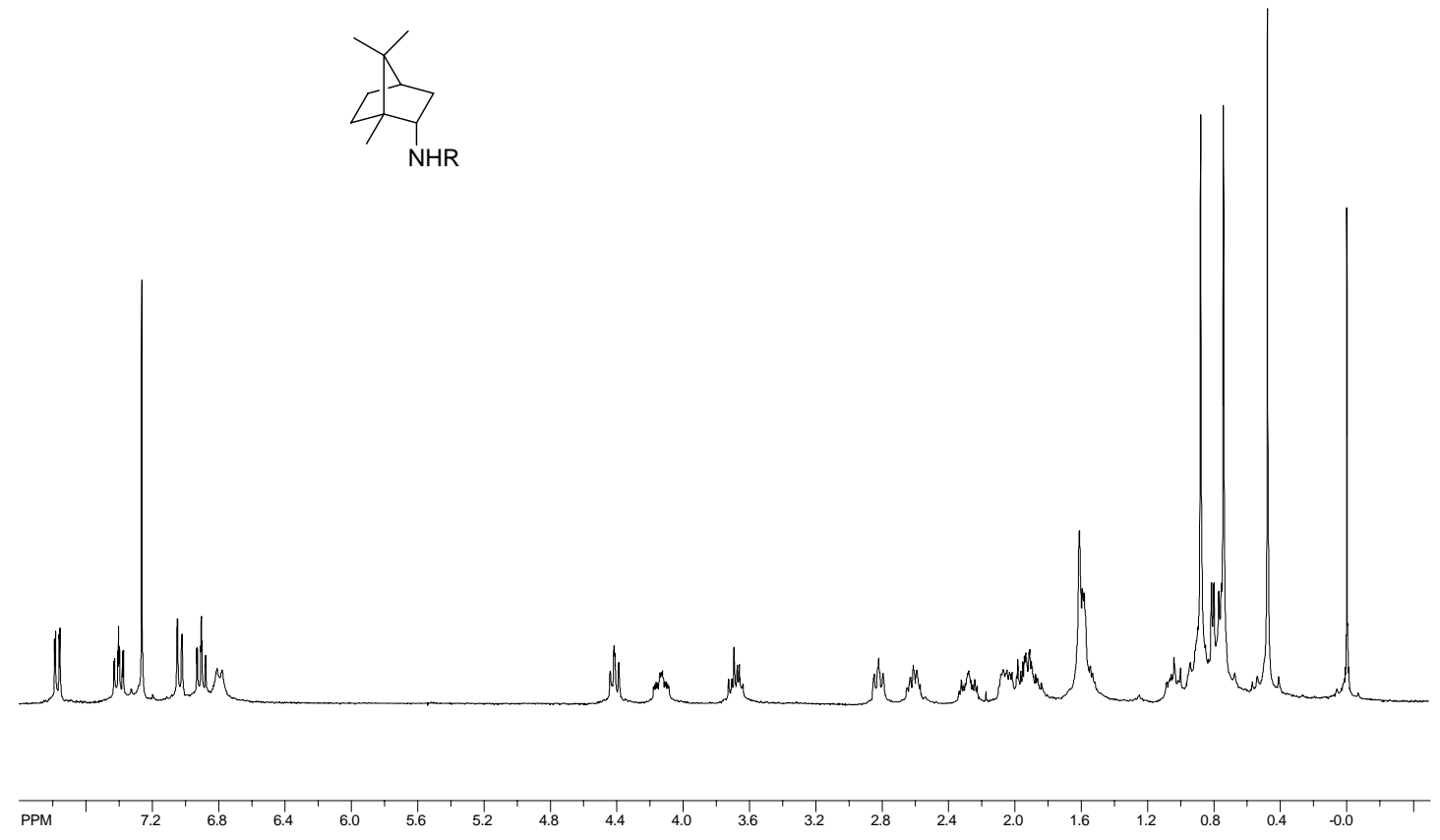

8, $\mathrm{R}=(R)-2-\mathrm{NPP}$

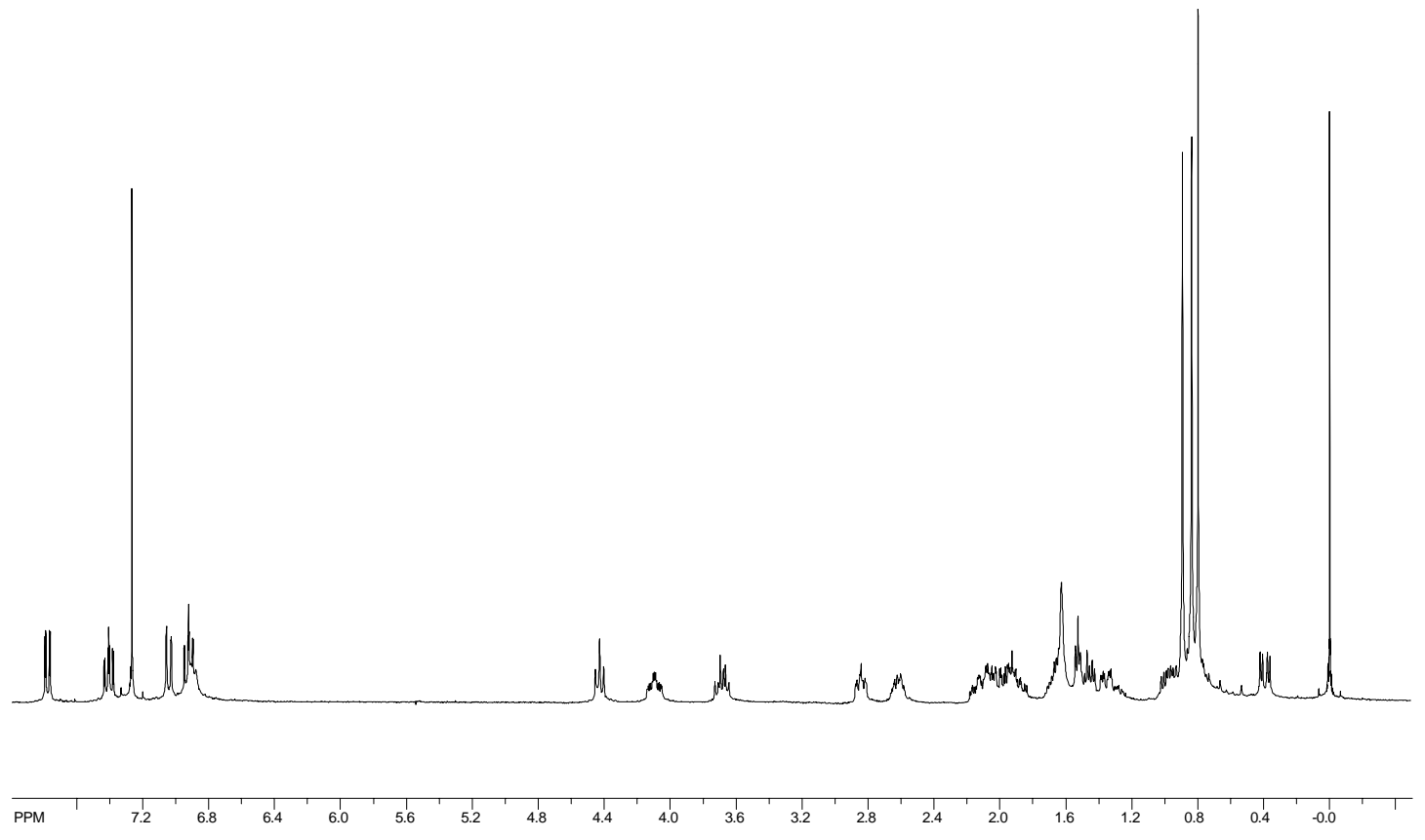

S19 
8, $\mathrm{R}=(S)-4-\mathrm{NPP}$
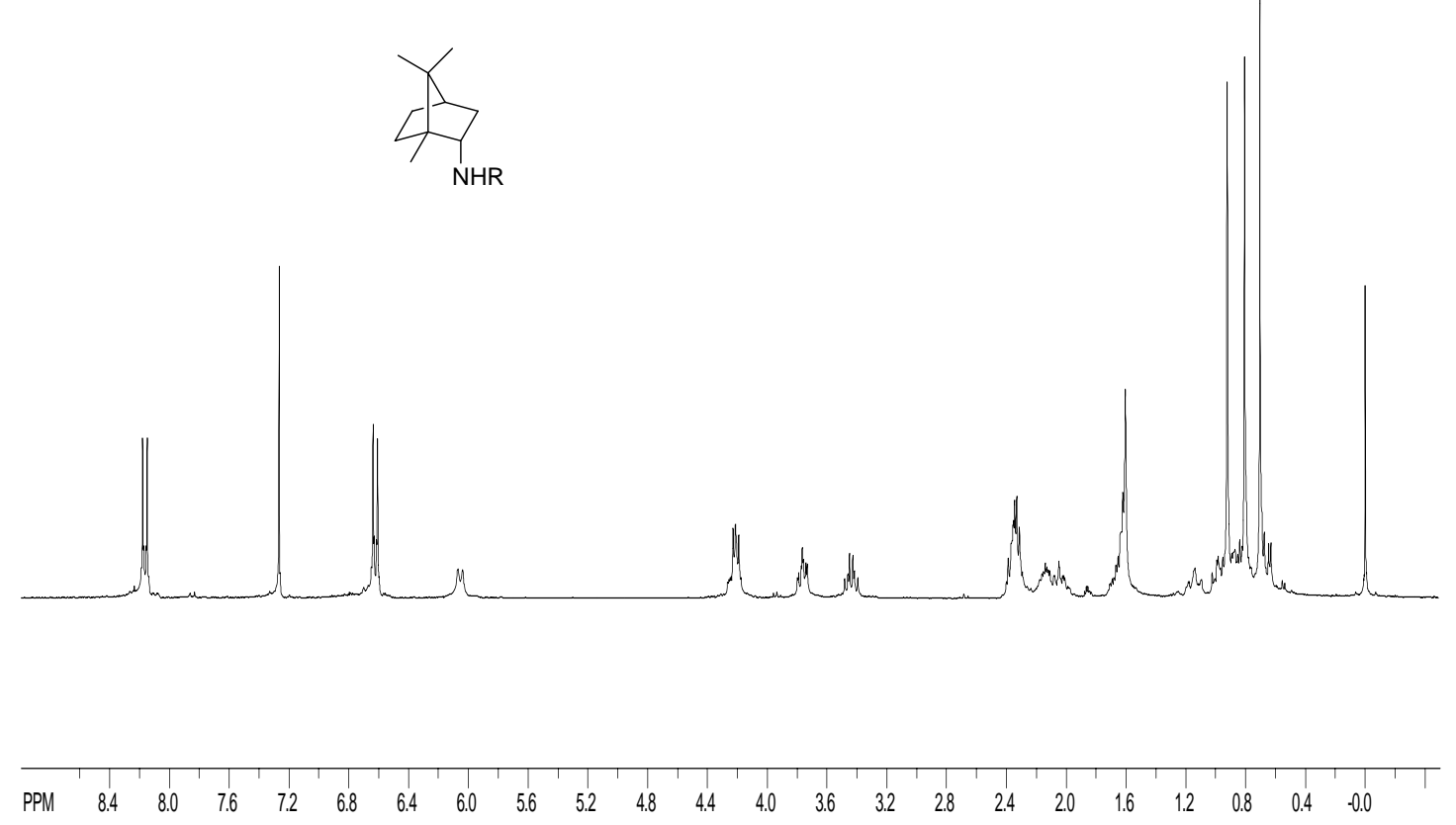

$\mathbf{8}, \mathrm{R}=(R)-4-\mathrm{NPP}$

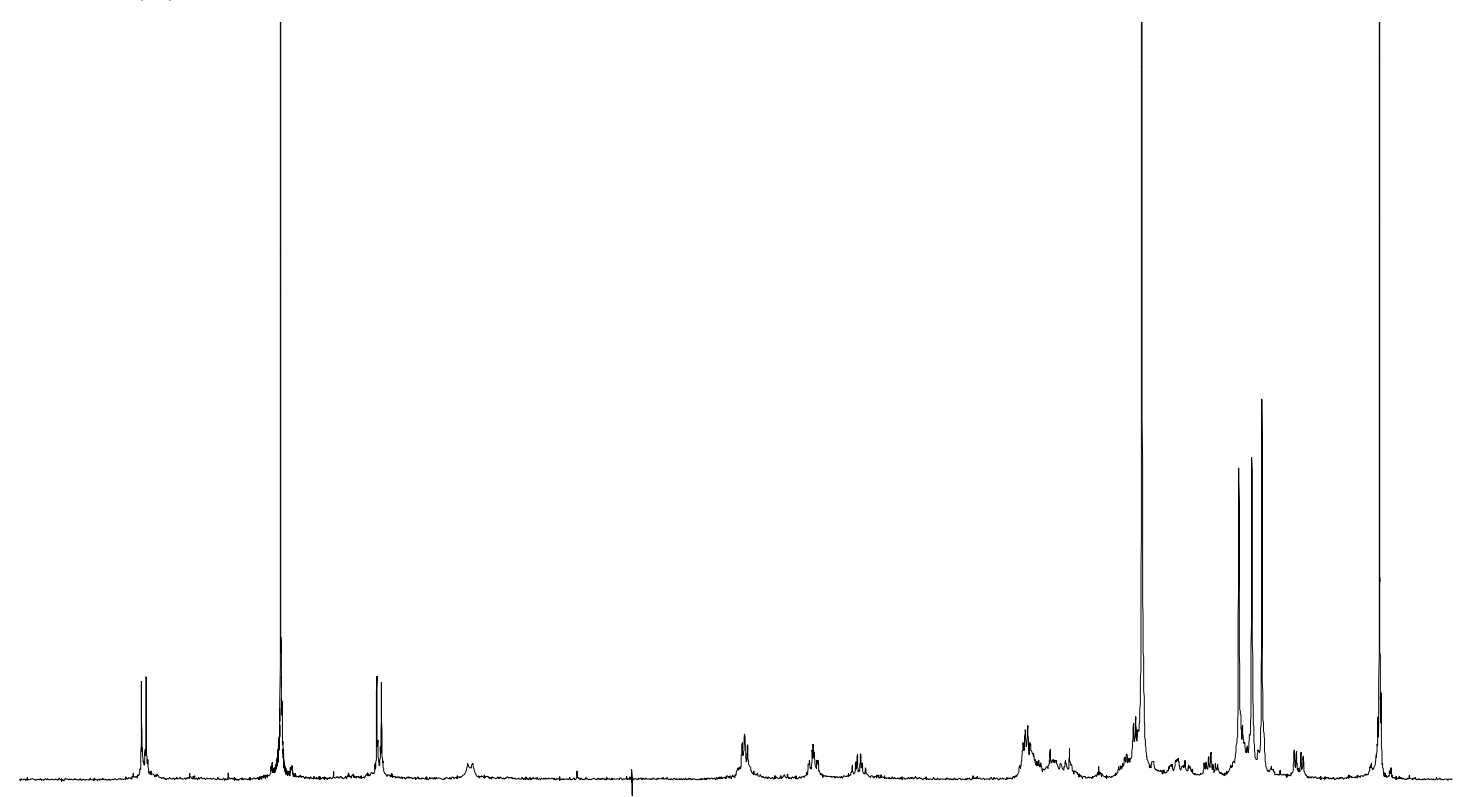


9, $\mathrm{R}=(S)-2-\mathrm{NPP}$
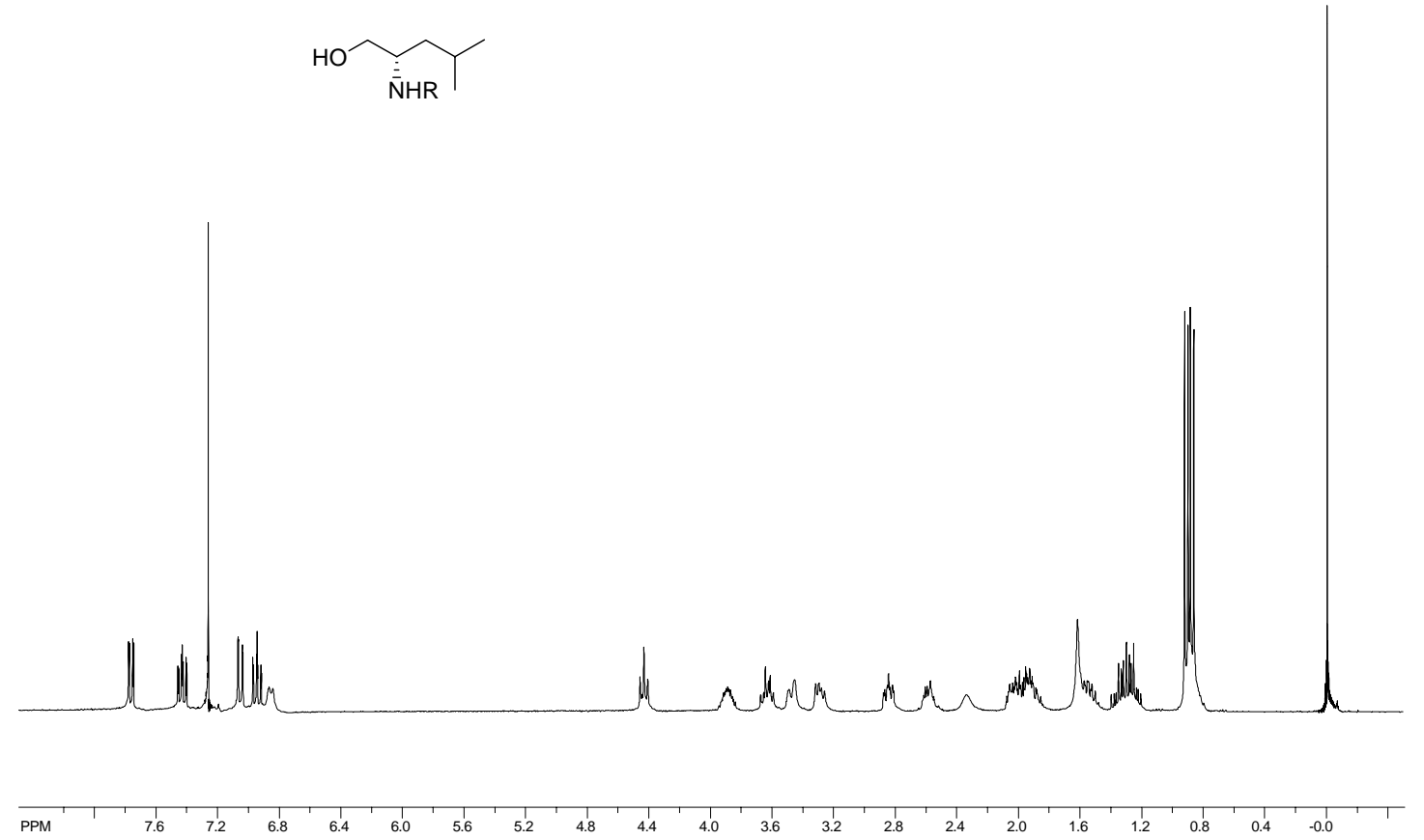

9, $\mathrm{R}=(R)-2-\mathrm{NPP}$

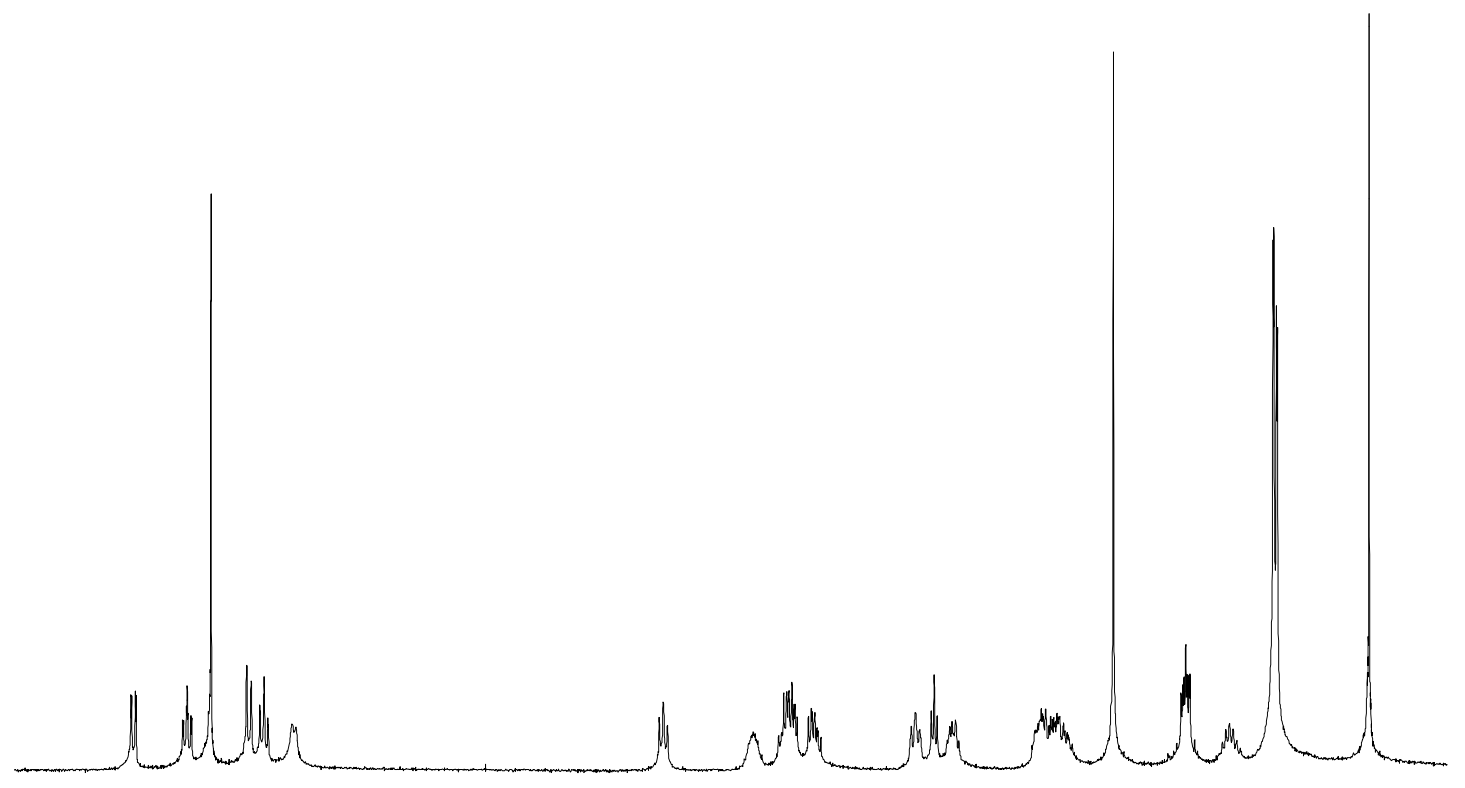

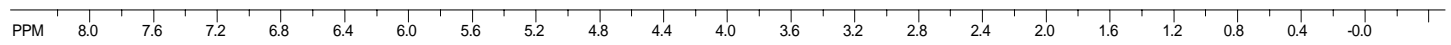


10, $\mathrm{R}=(S)-2-\mathrm{NPP}$

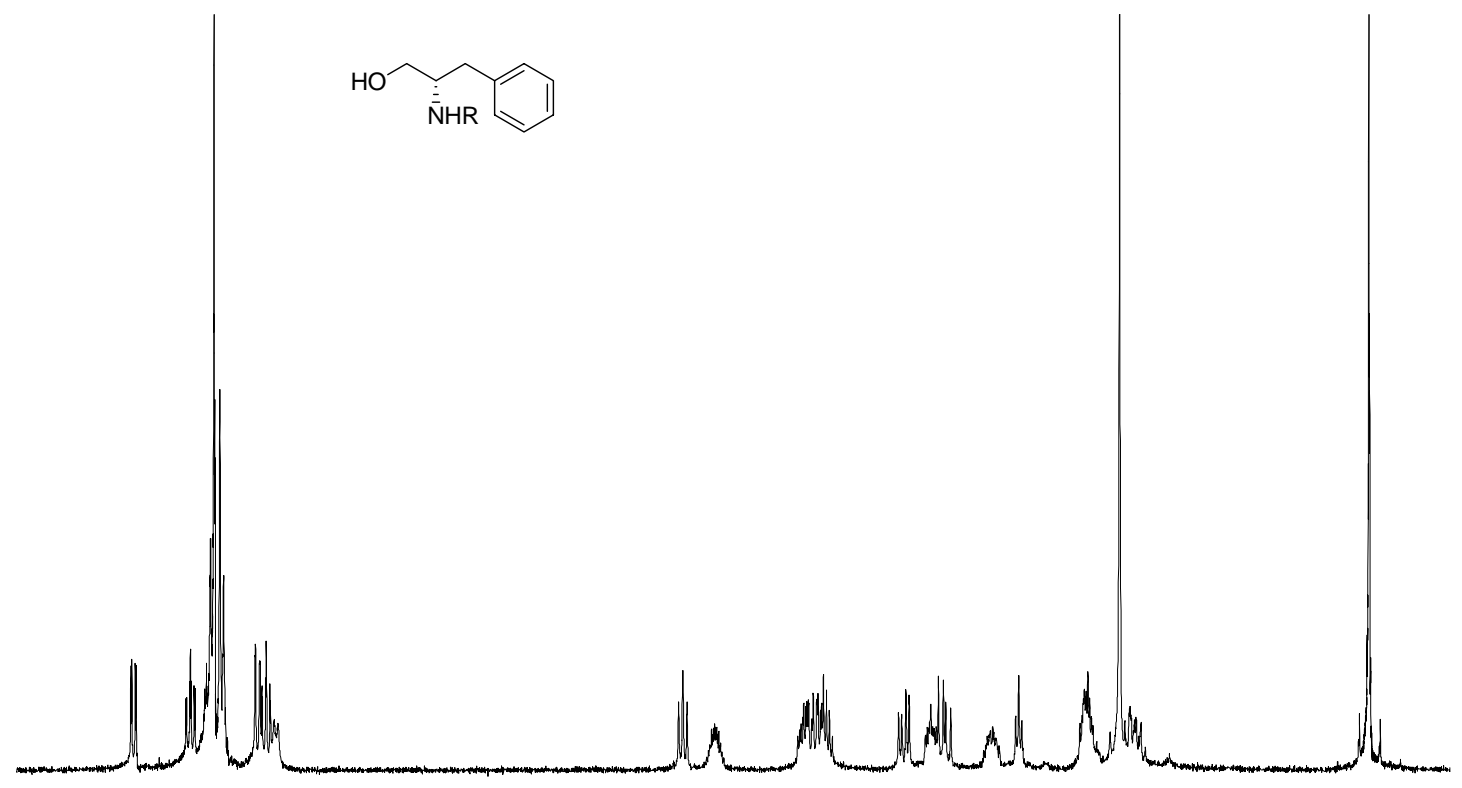

10, $\mathrm{R}=(R)-2-\mathrm{NPP}$

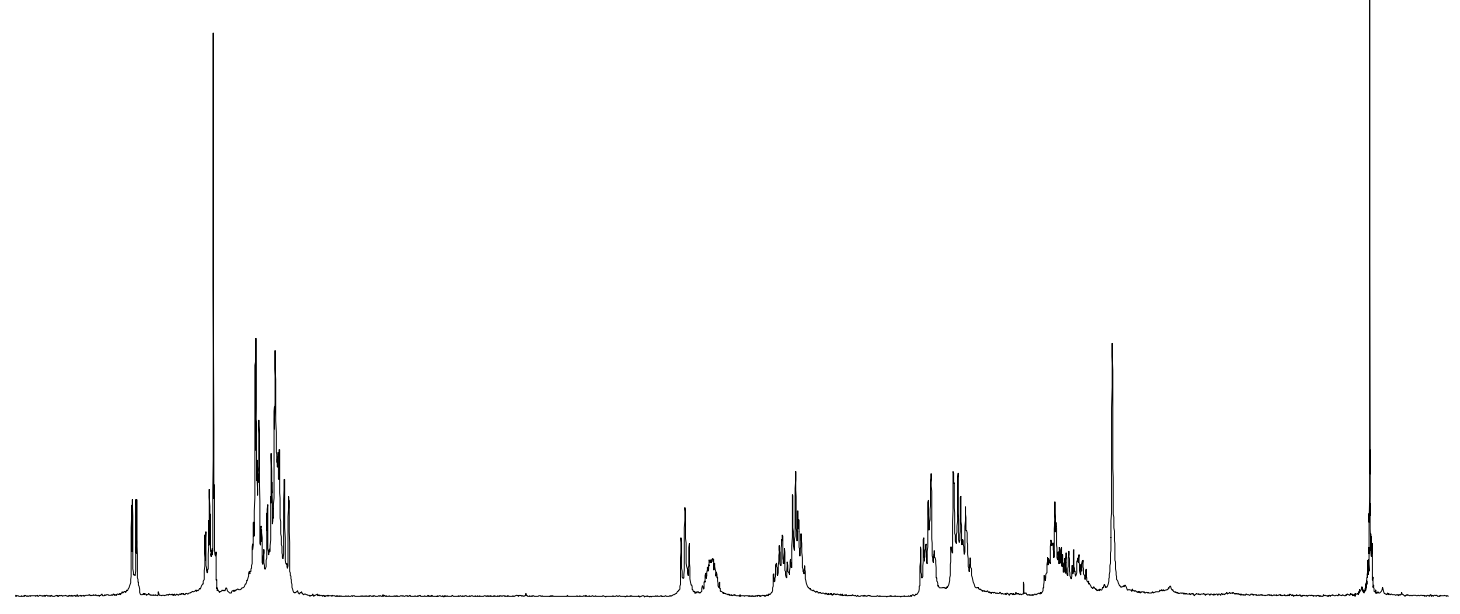

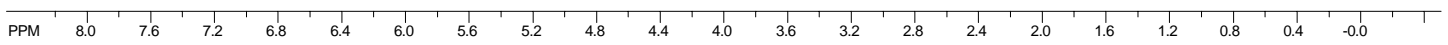


11, $\mathrm{R}=(S)-2-\mathrm{NPP}$
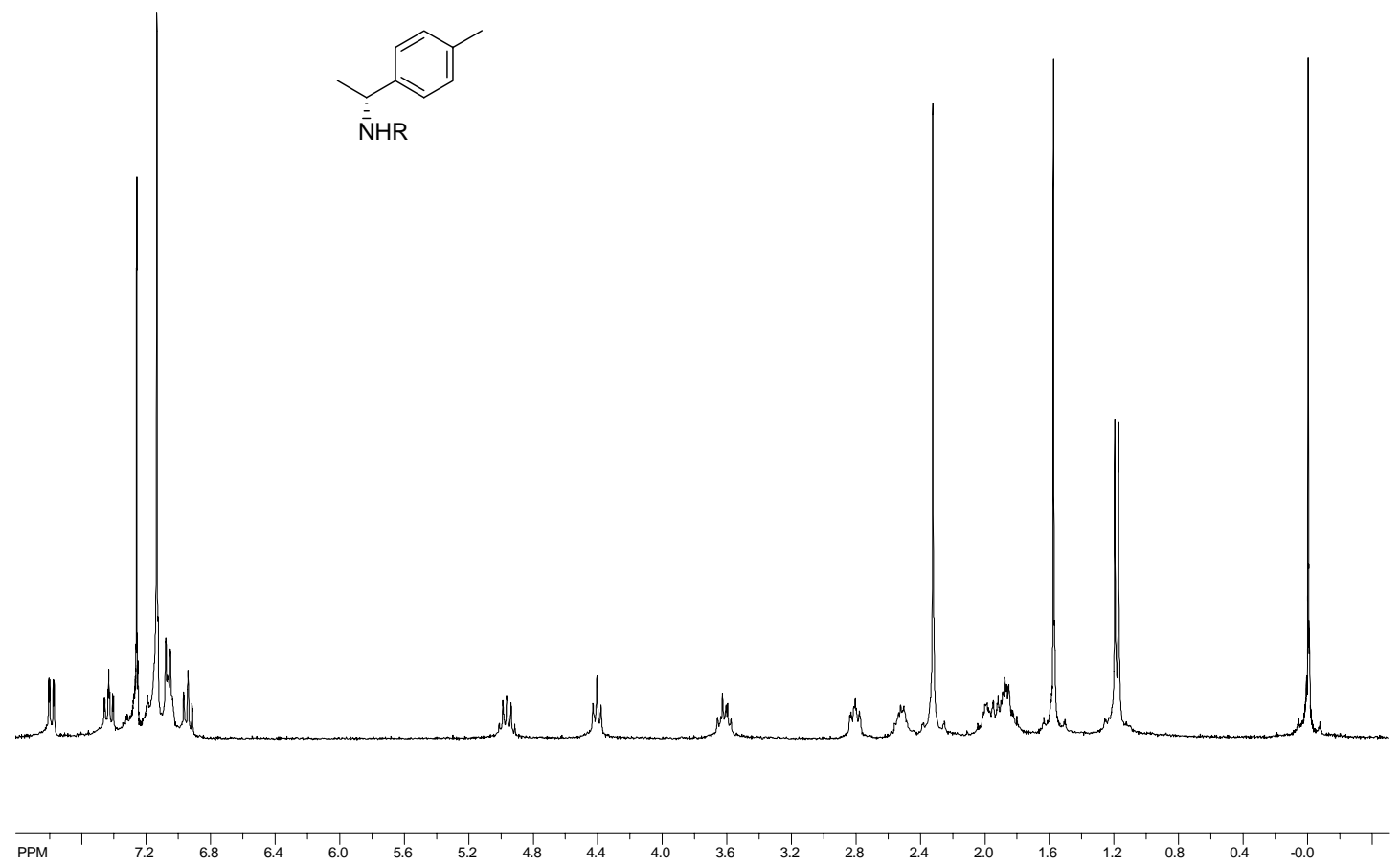

11, $\mathrm{R}=(R)-2-\mathrm{NPP}$

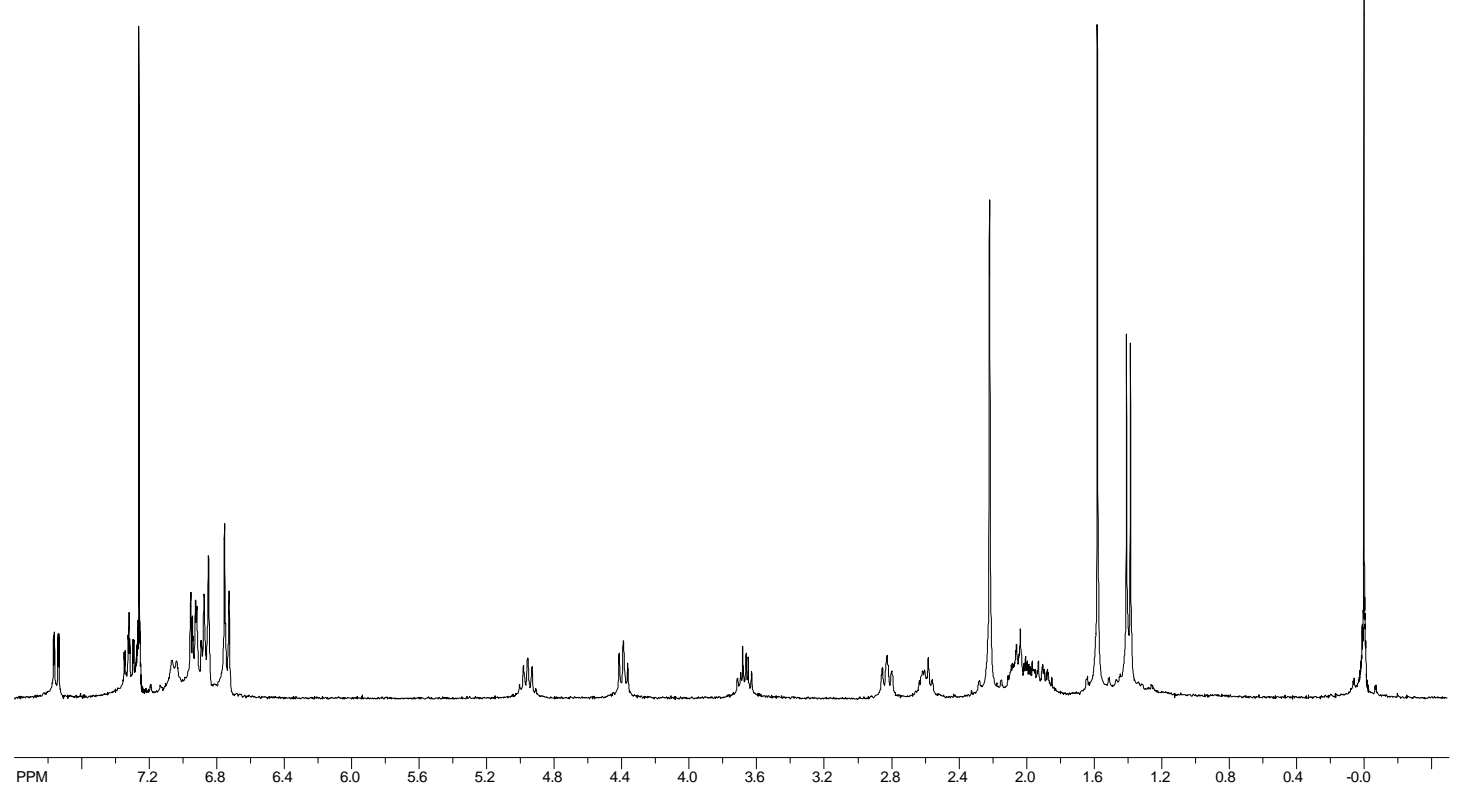


12, $\mathrm{R}=(S)-2-\mathrm{NPP}$
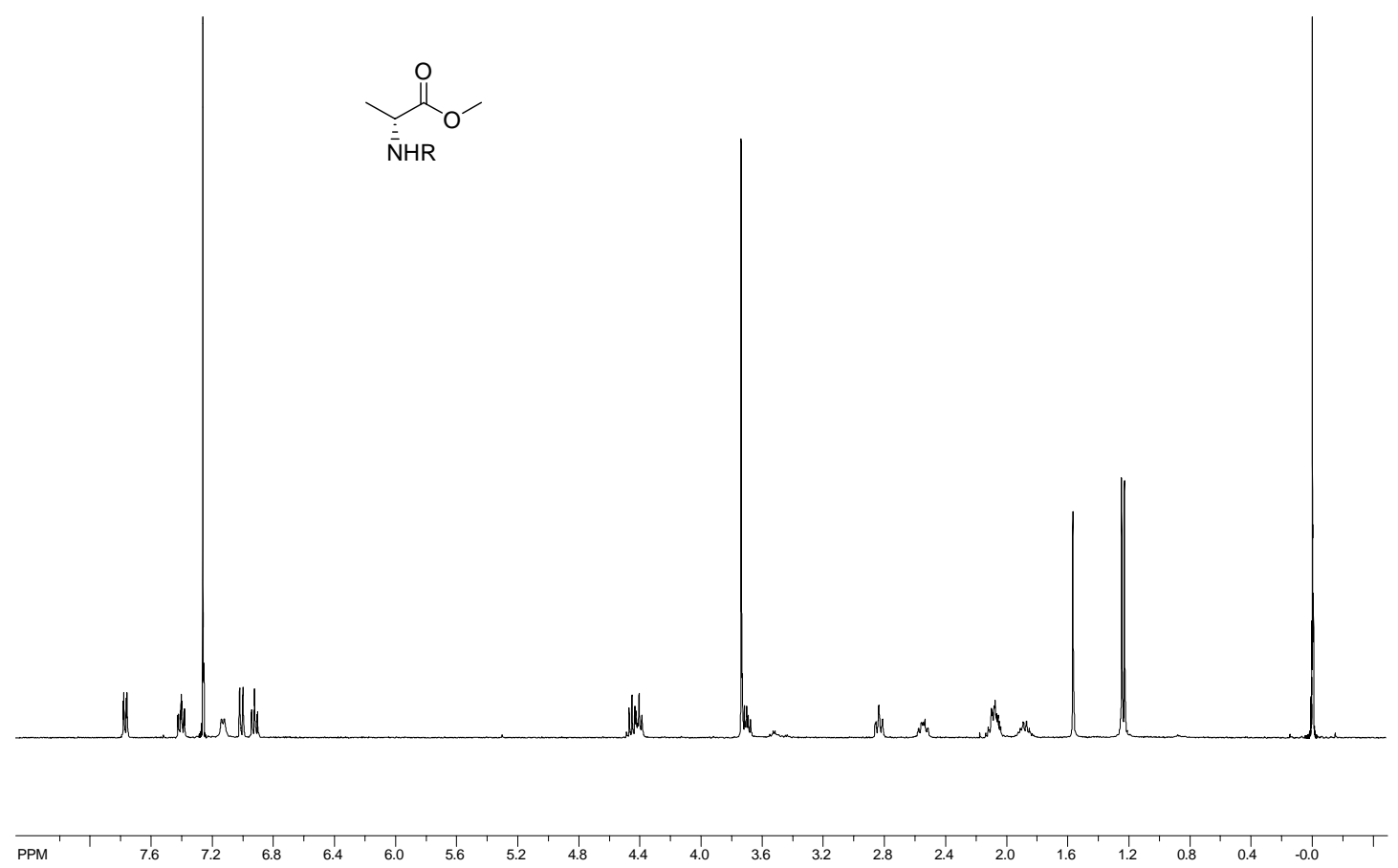

12, $\mathrm{R}=(R)-2-\mathrm{NPP}$

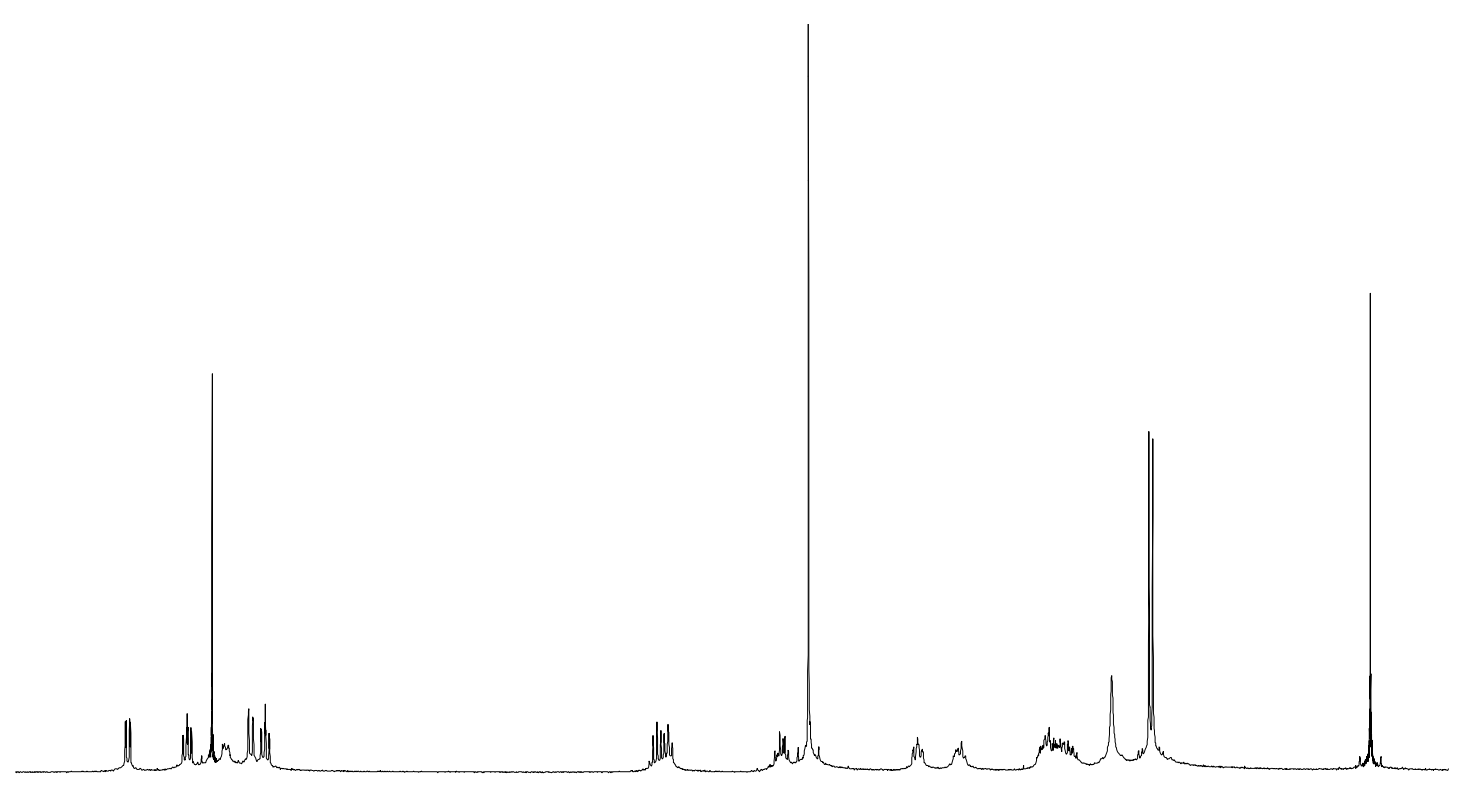

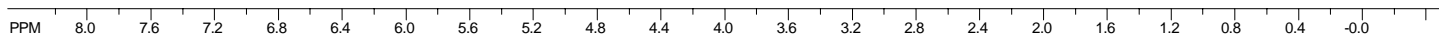


13, $\mathrm{R}=(S)-2-\mathrm{NPP}$
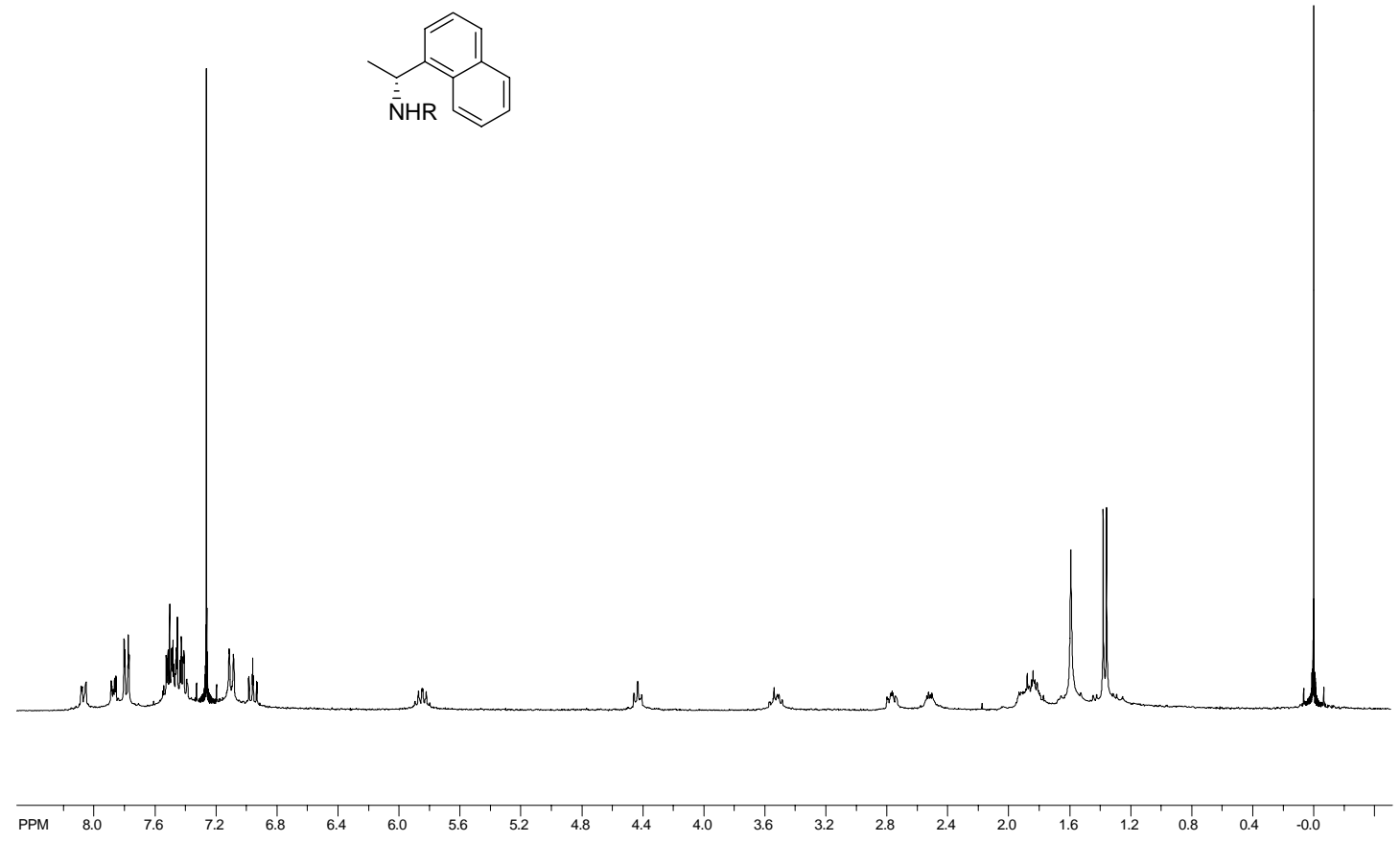

13, $\mathrm{R}=(R)-2-\mathrm{NPP}$

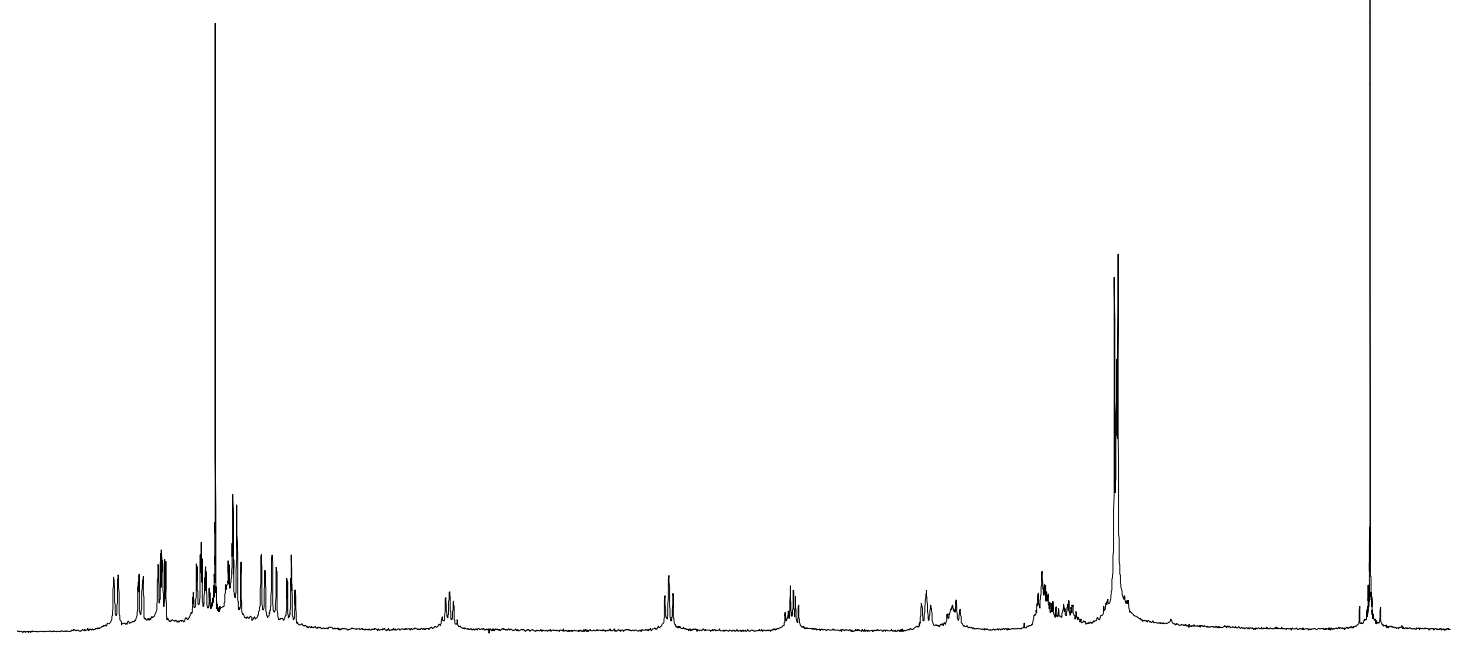

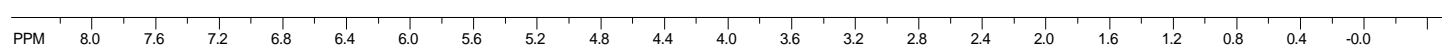


14, $\mathrm{R}=(S)-2-\mathrm{NPP}$
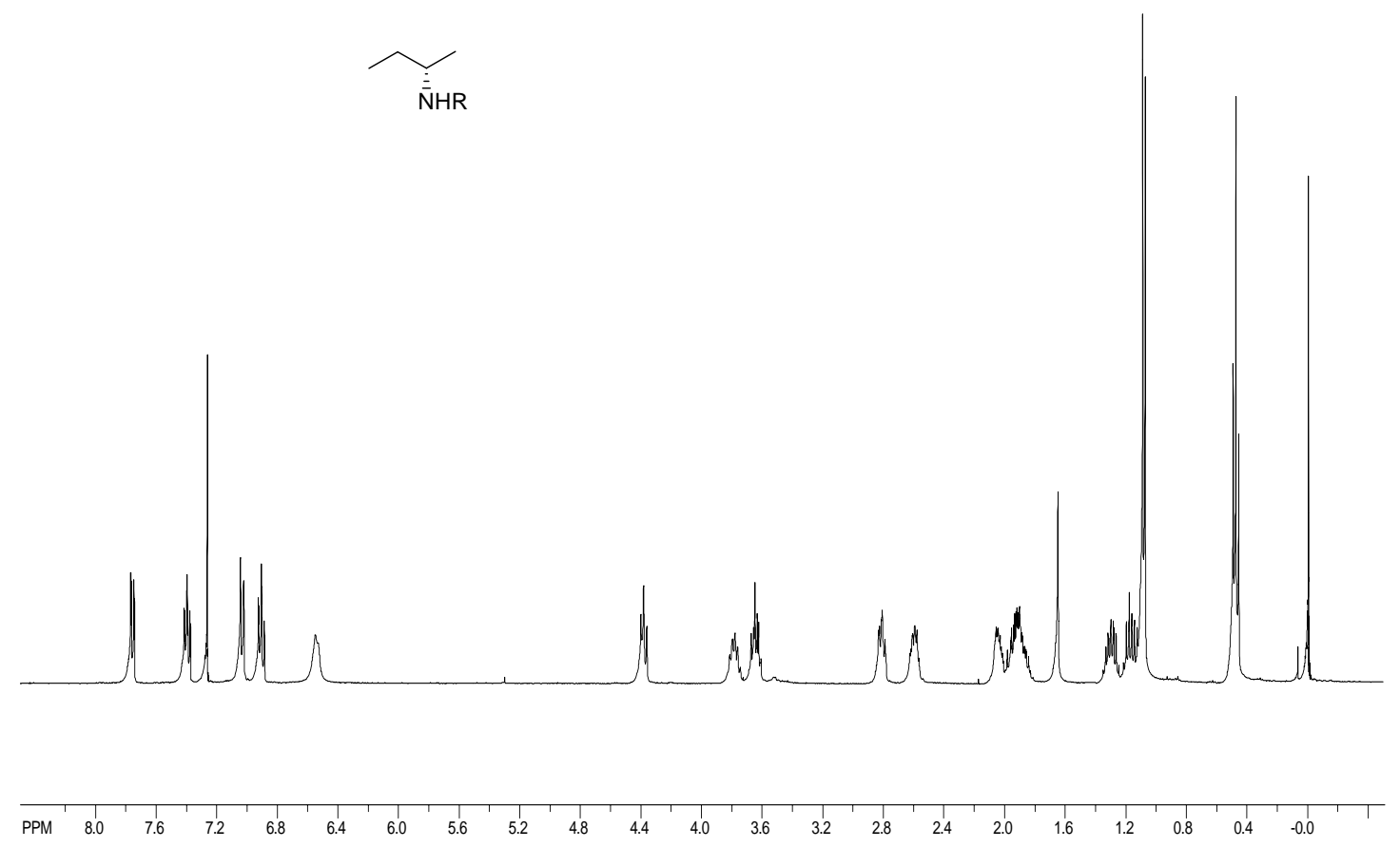

14, $\mathrm{R}=(R)-2-\mathrm{NPP}$

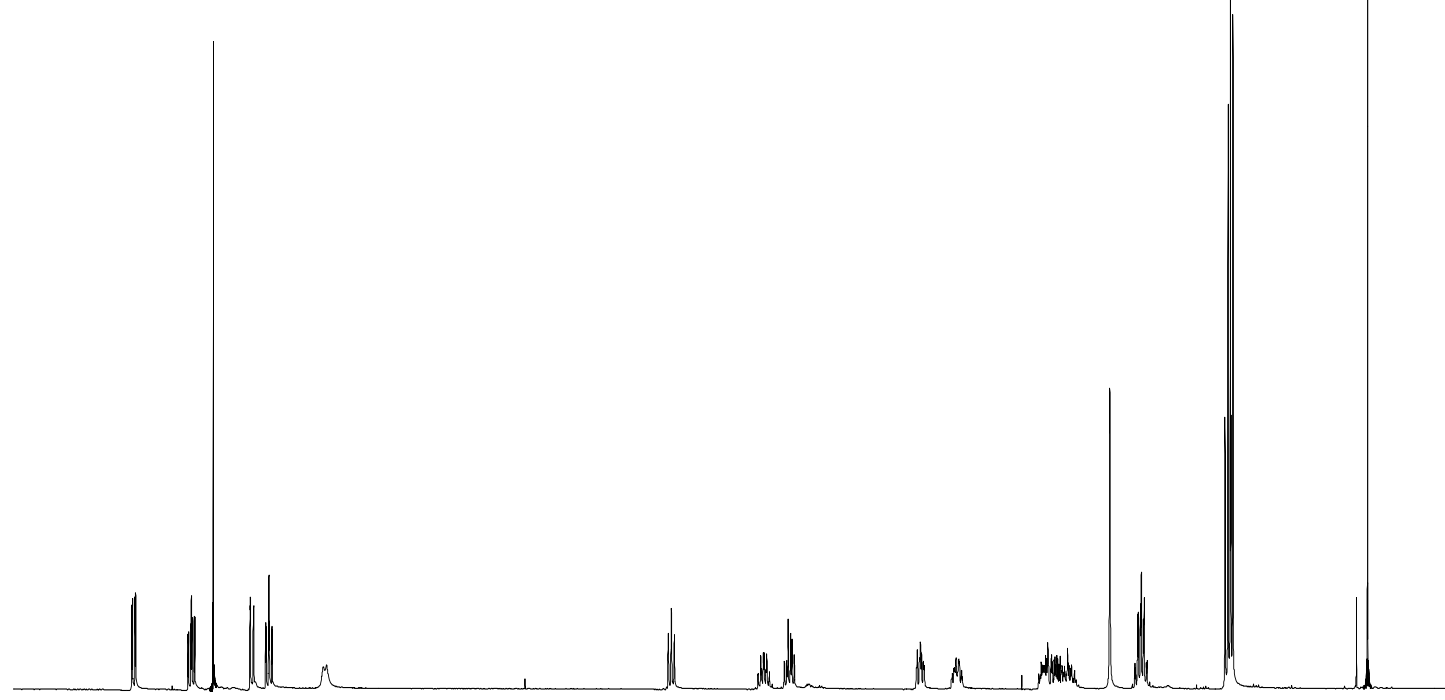

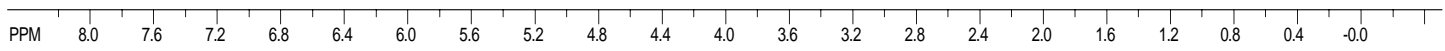

